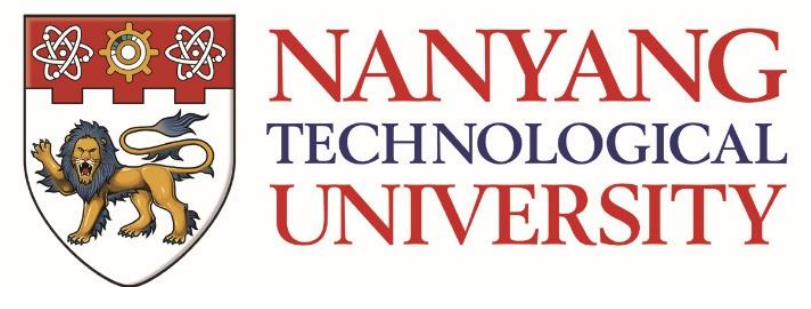

\title{
OPTICAL MICROFIBER REFRACTOMETRIC SENSORS
}

\author{
JI WENBIN
}

\section{NANYHANG TECHNOLOGICAL UNIVERSITY SCHOOL OF ELECTRICAL \& ELECTRONIC ENGINEERING}

A thesis submitted to Nanyang Technological University in partial fulfillment of the requirement for the degree of Doctor of Philosophy 


\section{Acknowledgement}

This work has come a long way and it will not have been possible without the support of many people. With this opportunity, I would therefore like to express my greatest appreciation towards them for their support and guidance they have given to me for the past 4 years.

First, I would like to thank my supervisor, Professor Tjin Swee Chuan, without whom I may encounter lots of problems that I cannot resolve alone. He is so brilliant in either technology side or school's administrative stuff. It is always a joy to listen to his talks on various occasions such as his physics class, the fortnightly meeting, seminars and so on. I would like to express my sincere appreciation for his consistent guidance and encouragement which inspired me in both my academic and daily life.

I would wish to thank my colleague Stephanie Yap Hui Kit, with whom I had a great experience in doing research. She is my junior and at first I taught her experimental procedures as well as some fundamental knowledge. But she was able to catch up very quickly and we learned and shared a lot in my last year of study.

I would like to thank Dr. Lin Bo, who is my senior, for his great help on my experiments at my initial phase of $\mathrm{PhD}$ life. With his help, I saved a lot of time in self-exploration on the use of various equipment. He shared a lot of his experiences regarding the issues that may arise during experiments and ways of resolving them. 
I would also like to express my appreciation to Associate Professor Yong Ken Tye, who acts like a co-supervisor of mine and constantly encourages me and shares his experiences in research. It is always a pleasure to learn from him.

I would also wish to give my thanks to the technical staff members in Photonics Lab 1 and 2, Mr. Tsay Chi Huang, Ms. Yee Yang Boey, Mr. Lim Teng Kheng, Ms. Low Poh Chee and Mr. Yong Kim Lam. Their patient support is important for me to carry out various experiments.

Last but not least, my greatest gratitude goes to my family members, especially my wife. You have sacrificed three years' career time to raise our two little children. You are always the most supportive one to me.

Thank you. 


\section{List of Figures}

Figure 1.1 Periodic wavelength response of interferometric sensor ............................ 14

Figure 2.1 Dependence of the spot size on the V number of a microfiber. The two troughs represent the points of maximum confinement in the cladding/core. [55]. 22

Figure 2.2 Dependence of the power fraction propagating in the evanescent field $\eta E F$ on

the normalized wavelength $\lambda / \mathrm{r}$ in different refractive index surroundings [55]

Figure 2.3 Schematic view of a taper profile

26

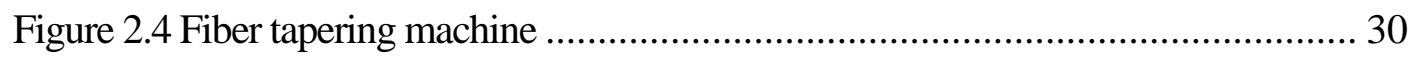

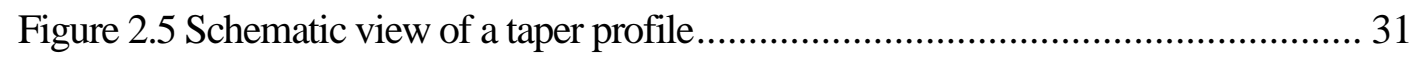

Figure 2.6 Microscopic view of an adiabatically-tapered optical fiber ........................ 32

Figure 2.7 Microscopic view of a non-adiabatically-tapered optical fiber ................... 32

Figure 2.8 Transmission spectra of an adiabatically tapered microfiber ..................... 33

Figure 2.9 Transmission spectra of a non-adiabatically tapered microfiber .................. 33

Figure 3.1 Electric field distribution of guided modes in an optical fiber (longitudinal

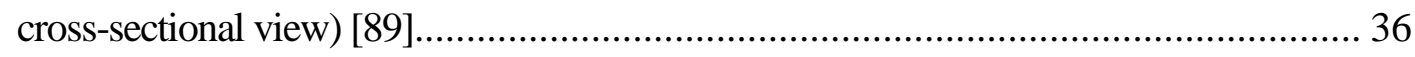

Figure 3.2 Graphic representation of fiber Bragg gratings................................... 37

Figure 3.3 Schematic diagram of a typical FBG setup ....................................... 40 
Figure 3.4 Experimental setup of fabrication of microfiber Bragg gratings

Figure 3.5 Schematic diagram of microfiber Bragg gratings 43

Figure 3.6 SEM image of the microfiber. 44

Figure 3.7 Reflection spectrum of an MFBG with a diameter of $7.6 \mu \mathrm{m}$ using single-mode fiber. 44

Figure 3.8 Wavelength versus the RI of the surrounding medium. 46

Figure 3.9 Reflection spectrum of MFBG with diameter of $7.5 \mu \mathrm{m}$ using multimode fiber

Figure 3.10 Reflection spectrum of MFBG with diameter of $6.4 \mu \mathrm{m}$ using multimode fiber 48

Figure 3.11 Schematic diagram of mode evolving in LPG 49

Figure 3.12 Schematic diagram of the experimental set-up 52

Figure 3.13 Refractive index profile of the W type double-clad fiber. 53

Figure 3.14 Wavelength response for different external refractive index $(D=60 \mu \mathrm{m})$ 56

Figure 3.15 Wavelength response for different external refractive index $(D=55 \mu \mathrm{m})$ 
Figure 3.16 RI sensitivity characterization for both sensors $(D=60 \mu \mathrm{m}$ and $D=55$ $\mu \mathrm{m})$ 57

Figure 3.17 Wavelength response for different temperature $(D=55 \mu \mathrm{m})$. 60

Figure 3.18 Temperature sensitivity characterization $(D=55 \mu \mathrm{m})$ 60

Figure 4.1 Plot of optical power coupling out of a microfiber against different taper diameter. The inset shows the E-field profile of fundamental mode of a microfiber with a taper waist diameter of $4 \mu \mathrm{m}$. 64

Figure 4.2 (a) SEM image of the taper waist with a diameter of $4.61 \mu \mathrm{m}$ and (b) experimental set-up of the refractive index sensor. 66

Figure 4.3 Reflection spectrum and transmission spectrum of a non-adiabatically tapered microfiber. 66

Figure 4.4 (a) Calculated results and (b) experimental results of wavelength response of the microfiber sensor surrounded by different concentration of the salt solutions. 68

Figure 4.5 Resonant wavelength shift as a function of RI: theoretical prediction and measured result for waist diameter $\sim 5.11 \mu \mathrm{m}$ 70

Figure 4.6 Resonant wavelength shift as a function of RI: comparison for two different taper diameters. 71

Figure 4.7 Wavelength responses for microfiber with diameter of $9.5 \mu \mathrm{m}$ in glycerol solutions with RI of 1.4064 and 1.4068 
Figure 4.8 Wavelength responses for microfiber with diameter of $4.8 \mu \mathrm{m}$ in glycerol

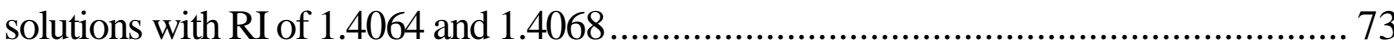

Figure 4.9 Wavelength responses for microfiber with diameter of $3.9 \mu \mathrm{m}$ in glycerol

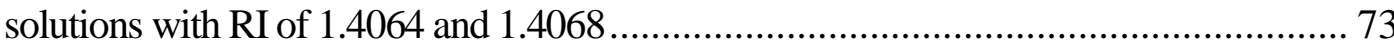

Figure 4.10 Sensitivity characterization with different diameters and a quadratic fitting.. 74

Figure 4.11 Setup of combining FBG and abruptly tapered microfiber as dual sensor .... 75

Figure 4.12 A FBG sensor. The refractive index change in the surrounding area of the

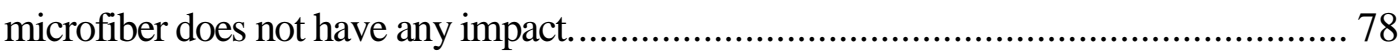

Figure 4.13 A refractive index sensor spectrum. The FBG peak will not have impact..... 79

Figure 5.1 The microfiber sensor with FSR of only $20 \mathrm{~nm}$ 81

Figure 5.2 Wavelength response of abrupt fiber taper with short waist. The taper profile was set as $1 \mathrm{~mm}$ for upper taper, $2 \mathrm{~mm}$ for waist length, $2 \mathrm{~mm}$ for down taper. The diameters were set as 5

Figure 5.3 Wavelength response of abrupt taper with pulling speed of (a) $0.5 \mathrm{~mm} / \mathrm{s}$ (b) 0.2 $\mathrm{mm} / \mathrm{s}$, both the two taper's profiles were set as $1 \mathrm{~mm}$ for upper taper, $3 \mathrm{~mm}$ for waist

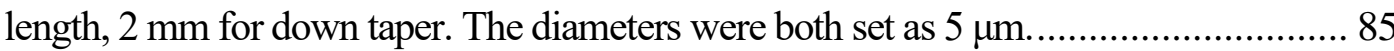

Figure 5.4 Experimental set-up and microscopic snapshots of the ultra-short non-adiabatic microfiber and SEM image of the microfiber waist 90 
Figure 5.5 Free spectral range (FSR) of microfibers with diameters of approximately $7 \mu \mathrm{m}$ for (a) Waist length=4.9 mm with $F S R=37 \mathrm{~nm}$, (b) waist length=4 $\mathrm{mm}$ with $F S R=48 \mathrm{~nm}$, (c) waist length=3.2 $\mathrm{mm}$ with $F S R=58 \mathrm{~nm}$, (d) waist length=2.4 $\mathrm{mm}$ with FSR=80 nm, (e)

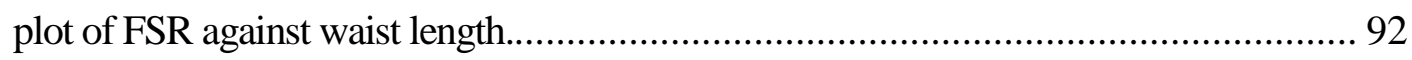

Figure 5.6. Wavelength responses (a) for natural evaporation of the glycerol solution droplets (The RI of the droplets increase 0.0002 for every 10 minutes) of microfiber with diameter $4.85 \mu \mathrm{m}$ and waist length $4.8 \mathrm{~mm}$. (b) for the microfiber in Fig. 5.4 (d) for different RI of glycerol solution. (Diameter $7.03 \mu \mathrm{m}$, waist length $2.4 \mathrm{~mm}$ ).

Figure 6.1 Surface functionalization of microfiber by silanization and the reaction scheme of EDC/NHS coupling for bonding of chelating agent onto the surface . 101

Figure 6.2 (a)-(c): SEM image with magnification $\times 5000$ of (a) silanized fiber using APTES (b) functionalized fiber with EDTA attached on the surface and (c) functionalized fiber with lead (II) ion trapped on the surface by EDTA............. 102

Figure 6.3 The possible silanization process of microfiber surface 104

Figure 6.4 (a)-(c) The spectra of microfiber sensor obtained from SEM-EDX analysis. Green color frame in the inset diagram represents the scanning area.... 105

Figure 7.1 Microfiber sensor system set-up. 108

Figure 7.2 Characterization of (a) Strain sensitivity (b) Temperature sensitivity 109 Figure 7.3 Sensitivity comparison between non-functionalized and functionalized microfiber sensors 111 
Figure 7.4 Sensitivity comparison between different chelating agents

Figure 7.5 Wavelength responses for DI water and $10 \mathrm{ppb}$ copper (II) ion solution using microfiber sensor with diameter of $3.9 \mu \mathrm{m}$ coated with DPA.................... 115 


\section{List of Acronyms}

ABS

APTES

DI

DL

DPA

DWDM

EDC

EDTA

EDX

EMI

FBG

FHB

FSR

LPG

MF

MFBG

MLPG

MMF

MNF

MZI
Acrylonitrile Butadiene Styrene

Aminopropyltriethoxysilane

De-ionized

Detection Limit

D-Penicillamine

Dense Wavelength Division Multiplexing

Carbodiimide Hydrochloride

Ethylenediaminetetraacetic Acid

Energy-dispersive X-ray spectroscopy

Electromagnetic Interference

Fiber Bragg Gratings

Fiber Holding Block

Free Spectral Range

Long Period Gratings

Microfiber

Microfiber Bragg Gratings

Microfiber Long Period Gratings

Multi-mode Fiber

Microfiber and Nanofiber

Mach-Zehnder interferometer 
NHS

OSA

PCF

PDMS

RI

SEM

SMF

TLS

WHO
N-hydroxysuccinimide

Optical Spectrum Analyzer

Photonic Crystal Fibers

Polydimethylsiloxane

Refractive Index

Scanning Electron Microscopy

Single-mode Fiber

Tunable Laser Source

World Health Organization 


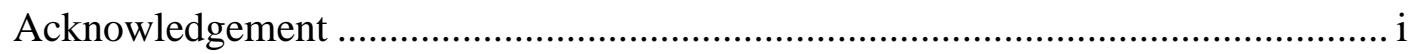

List of Figures .......................................................................................... ii

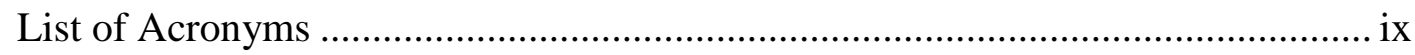

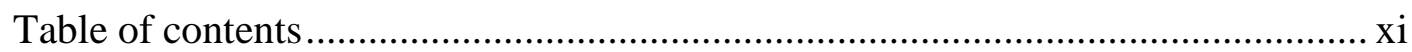

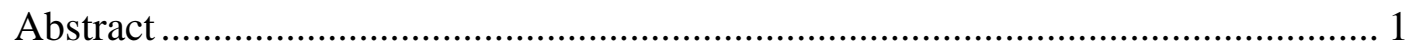

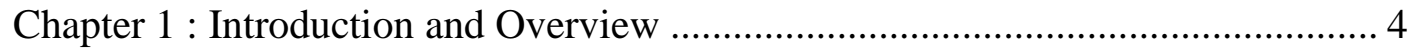

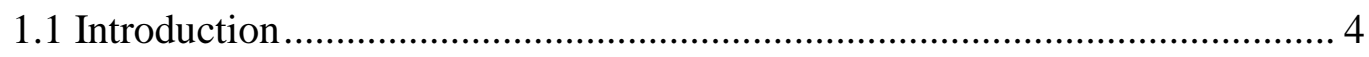

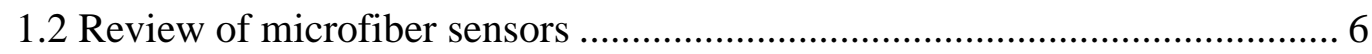

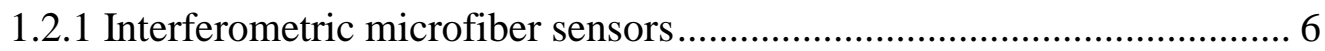

1.2.2 Gratings assisted microfiber sensors ........................................ 8

1.2.3 Surface Plasmon Resonance based microfiber sensors ....................... 10

1.2.4 PCF microfiber sensors .......................................................... 11

1.2.5. Other types of microfiber sensors ............................................. 12

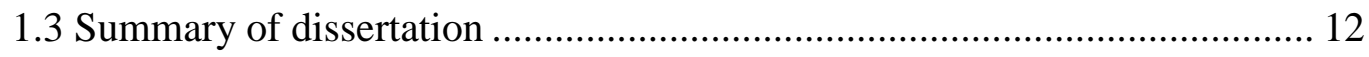

1.4 Outline of dissertation...................................................................... 16

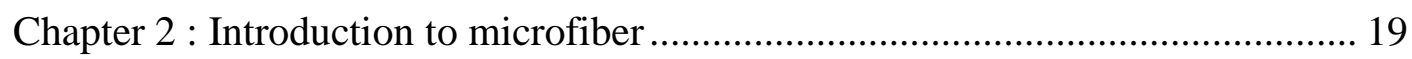

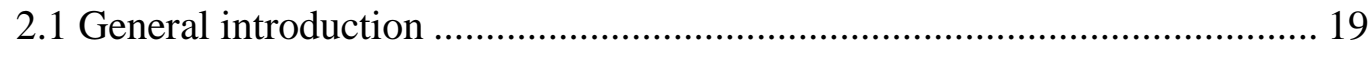

2.2 Optical waveguiding properties of microfiber ...................................... 19 


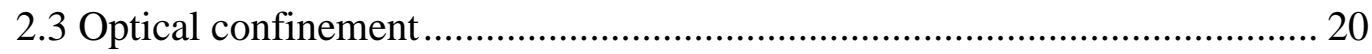

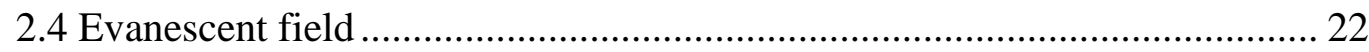

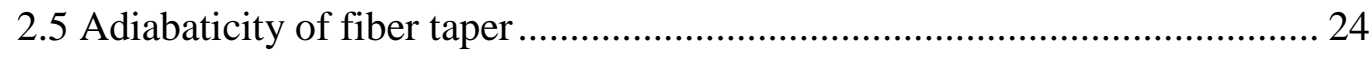

2.5.1 Length-scale criterion .......................................................... 25

2.5.2 Weak power transfer criterion .................................................. 26

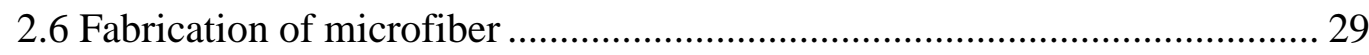

Chapter 3 : Adiabatically tapered microfiber sensors .................................... 35

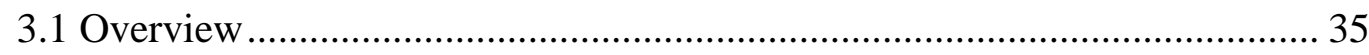

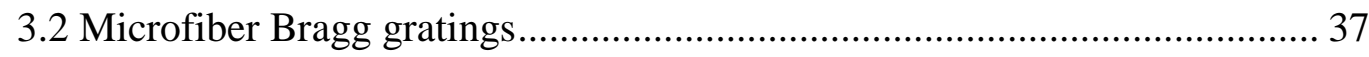

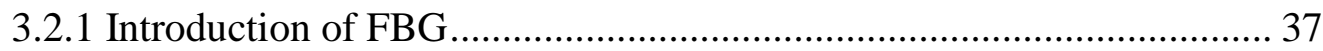

3.2.2 Microfiber Bragg gratings .................................................... 42

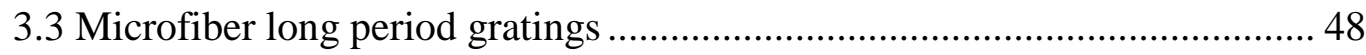

3.3.1 Introduction of long period gratings .......................................... 48

3.3.2 Experimental results.............................................................. 51

Chapter 4 : Non-adiabatically tapered microfiber sensors ............................... 61

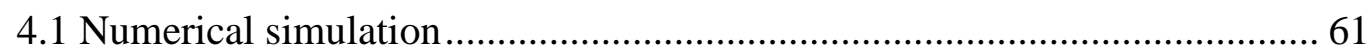

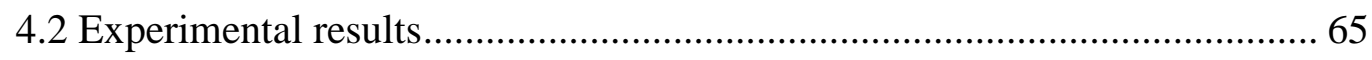

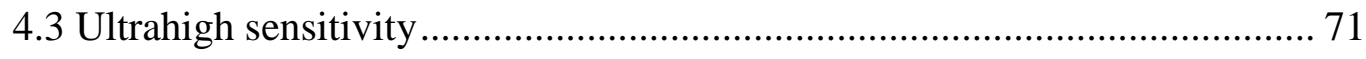

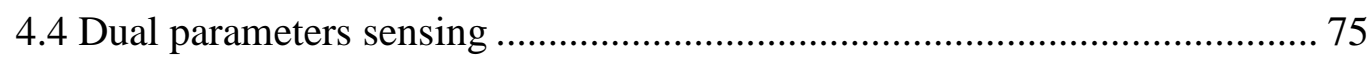


Chapter 5 Optimization of type 2 microfiber sensor 80

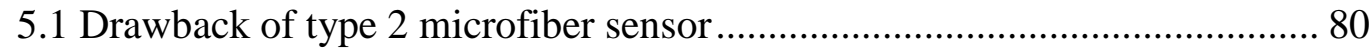

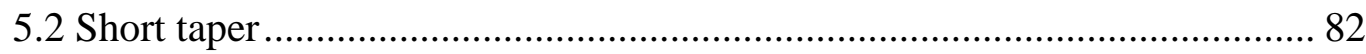

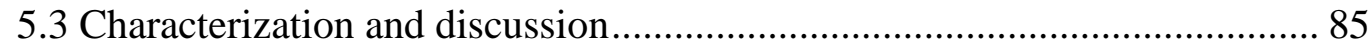

Chapter 6 Detection of heavy metal ions using type 2 microfiber sensor ............... 96

6.1 Overview of techniques for detecting heavy metal ions ............................... 96

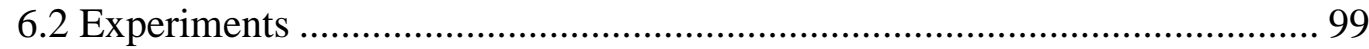

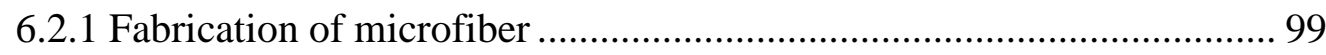

6.2.2 Microfiber surface modification .......................................................... 100

6.3 Scanning Electron Microscope (SEM) imaging ......................................... 101

Chapter 7 : Characterization of surface functionalized microfiber sensor............. 106

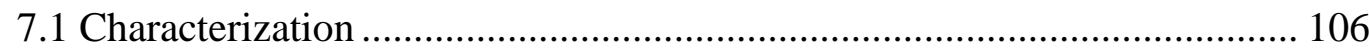

7.2 Wavelength responses for non-functionalized and functionalized microfiber

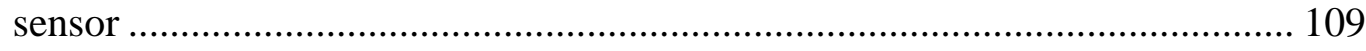

7.3 Comparison of coating different chelating agents ....................................... 111

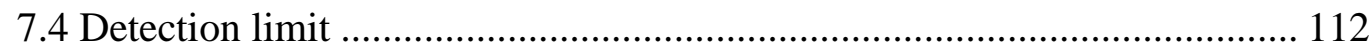

Chapter 8 : Conclusion and recommendation for future work .............................. 117

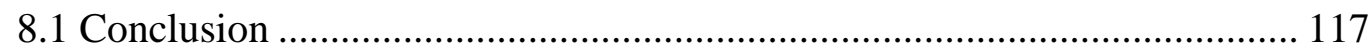

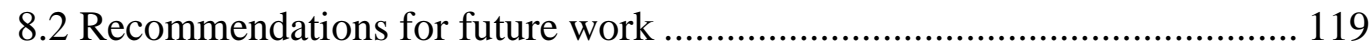

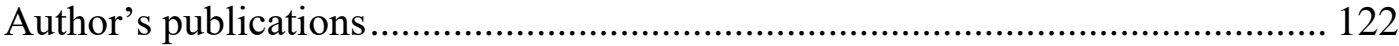




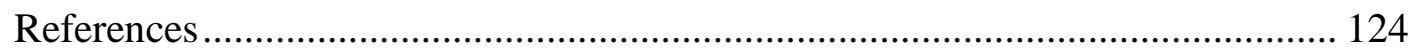




\begin{abstract}
In this study, the author has investigated two types of microfibers, namely the adiabatically (gradually) tapered and non-adiabatically (abruptly) tapered microfibers. For the adiabatic microfiber, the fiber is tapered gradually so that only the fundamental mode will propagate inside the fiber. Thus, the transmission spectrum of such microfiber is as flat as before it was tapered. Hence to use this type of fiber as a refractive index sensor, there is a need to incorporate other techniques in the microfiber to generate a reference wavelength such as resonant dip or peak so that the wavelength response due to external refractive index variation can be interrogated. In this project, the author has adopted two ways for doing this. One is to inscribe a fiber Bragg grating (FBG) and another is to inscribe a long period grating (LPG) in tapered portion of the photosensitive fiber. Both these two gratings were written into the taper waist portion using a $\mathrm{KrF}$ eximer laser. There is a wavelength peak in the reflection spectrum of microfiber Bragg grating (MFBG) and a wavelength dip in the transmission spectrum of microfiber long period grating (MLPG). The resonant peak and dip shift when the external refractive index changes. The RI sensitivity of MFBG was found to be moderate ( $~ 80 \mathrm{~nm} / \mathrm{RIU})$ as compared to other RI sensors while the sensitivity of MLPG ( 600 nm/RIU) was much higher than normal LPG RI sensors.
\end{abstract}

The other type of microfiber is the non-adiabatic microfiber, where the fiber taper slope is steep. As a result, higher-order modes are excited as light propagates down the fiber. The induced higher-order modes will interfere with the fundamental mode 
resulting in a sinusoidal wavelength response. The wavelengths will shift as the external refractive index (RI) increases. In the experiment, the diameter of the microfiber was reduced up to $4.6 \mu \mathrm{m}$ and the sensitivity was found to be extremely high, of around 50,000 nm/RIU. The author also has developed a numerical model that can predict the behavior of such non-adiabatically tapered microfiber. It is also worth mentioning that generally the smaller diameter microfiber gives a higher sensitivity as more evanescent field will be coupled to the surrounding medium. For the adiabatic microfiber sensors, although a smaller diameter is shown to be more sensitive, it is difficult to obtain very small diameters of few micrometers as the taper transitions need to be sufficiently long to maintain single mode in the microfiber. And since the abruptly tapered fiber can be fabricated with a much smaller diameter, it is thus easier to obtain higher sensitivity with it as compared to the gradually tapered fiber.

One common issue in interferometric based sensors is the free spectral range (FSR), as such sensors will not work properly once the resonant wavelengths shift over one cycle of the FSR. For tapered microfiber, it was found that the temperature and the pulling speed during the tapering process are the crucial control parameters to achieve the desired tapered profile. By setting a cooler tapering temperature and fast pulling speed, ultra-short microfibers with waist length of only $2.4 \mathrm{~mm}$ and total length of $5.5 \mathrm{~mm}$ were successfully fabricated. The resulting FSR is around $80 \mathrm{~nm}$ and the maximum sensitivity achieved for such short microfiber is $25,667 \mathrm{~nm} / \mathrm{RIU}$.

The above optimization work for the abruptly tapered microfiber sensor makes such sensor very useful in real applications. Heavy metal pollution in natural water 
environment is always a concern in both human health and our environment. All current systems available in the market for detecting heavy metal ions have common drawbacks such as bulky size, expensive set-up and costly maintenance. As a wrap up of my work, the author also presented an application of the optical microfiber sensor functionalized with chelating agent to detect the existence of specific heavy metal ions in water. A clear spectral shift was observed when the sensor was immersed into metal ion solution, and the minimum resolution achieved for copper ion can be as low as 10 parts per billion (ppb), utilizing a $3.9 \mu \mathrm{m}$ diameter fiber functionalized with the chelating agent D-Penicillamine (DPA). 


\section{Chapter 1 : Introduction and Overview}

\subsection{Introduction}

In past decades, sensing instruments in many applications such as civil engineering, environment and biomedical engineering etc are mainly electrical in nature. Despite their popularity, electrical sensors suffer from numerous drawbacks such as sensitivity to electromagnetic interference (EMI) and lack of multiplexing capability. To shield these sensors from EMI, the normal copper electrical cables have to be replaced with special shielded cables. In recent years, a lot of research has been done on optical fiber based sensors, which have shown potential to overcome some of the problems faced by electrical based sensors, owing to the well-known advantages of optical fiber, such as immunity to EMI, compact size and capability to implement in hazardous environment. Optical fiber sensors have been investigated for over a few decades due to these intrinsic advantages. After such a long development history, a lot of techniques have become mature. Some has already been commercialized and deployed in civil, environmental, biomedical and other engineering applications [1-3].

Grating-based optical fiber sensors are popular sensing techniques that were studied for over a few decades. There are two main types of grating-based optical fiber sensors, namely short period or Bragg gratings, and transmission-based or long period gratings. Fiber Bragg grating (FBG) sensors operate based on changing the optical properties of the back reflection of light within the core of the fiber, which results in the change of back-reflected wavelength. FBG sensors have been implemented for various types of perturbation monitoring such as strain, temperature [4] and refractive index [5]. The principle of a long period grating (LPG) 
sensor is based on the change of optical property of the forward propagating cladding modes. An LPG consists of a uniform modulation of the refractive index, with grating periods between $100 \mu \mathrm{m}$ and $1000 \mu \mathrm{m}$, within the optical fiber core. This modulation of refractive index results in the coupling of fundamental forward propagating core mode into discrete forward propagating cladding modes, in which the latter is sensitive to external perturbations.

Nowadays researchers have shown great interest in micro scale based optical fiber sensors. A microfiber is fabricated by placing the normal-sized fiber on a filament or flame that generates high heat to melt the fiber while pulling the fiber at both ends. Besides the properties such as high nonlinearity and low-loss interconnection to normal single-mode fiber, the large evanescent field of these microfibers plays an important part for refractive index sensing.

In this chapter, the author will summarize the popular optical microfiber based sensors reported in the recent decades, especially focusing on the refractive index sensing and their applications in biomedical and chemical areas. Refractive index (RI) sensing is a prominent subject of optical sensing that has a broad range of uses including the inspection of solute concentration in aqueous solutions and quality control in the monitoring in the food processing steps. The compact form of microfiber-based optical sensors makes sensing RI in microfluidic channels and water environments highly feasible and robust. In the following sections, the author will categorize the types of microfiber sensors that had been popularly studied by various researchers in the recent years. 


\subsection{Review of microfiber sensors}

The first experiment of microfibers in RI sensing application began as early as 1986, when Lacroix et al. [6] studied the transmitted core-mode power as a function of external RI with varying taper lengths. In 2005, Polynkin et al. [7] reported a simple microfiber based RI sensor consisting of a submicron-diameter optical fiber taper immersed in a transparent curable soft polymer. Liquid analyte surrounded the microfiber waist with length of around $3 \mathrm{~cm}$, making the optical loss sensitive to the RI difference between the polymer and the liquid. The detection limit was estimated to be $5 \times 10^{-4}$ RIU. Kude et al. [8] demonstrated another way to fabricate microfibers for RI sensing through chemical-etching. The lengths of the microfiber range from $0.5 \mathrm{~cm}$ to $1 \mathrm{~cm}$, and uniform waist diameters range from $6 \mu \mathrm{m}$ to $18 \mu \mathrm{m}$. Sui et al. [9] showed an alternative method for monitoring the RI-dependent optical transmission by measuring the phase change via a differential frequency technique. Their setup had shown a minimum detectable RI variation of $\sim 10^{-5}$ for RI ranges from 1.3322 to 1.334 . In the following sections, different types of microfiber sensor techniques will be described.

\subsubsection{Interferometric microfiber sensors}

Tian et al. [10] has firstly reported an in-line microfiber based Michelson interferometer, using a $500 \mathrm{~nm}$ thick gold coated end facet to reflect the core-mode and the abrupt-taper-induced cladding-mode that beats together at the tapered section. The microfiber has a diameter of $40 \mu \mathrm{m}$ and uniform waist length of $734 \mu \mathrm{m}$. A change in external RI produces a phase difference between the two paths of the modal interferometer that results in a wavelength shift of the interference fringes. 
The minimum detectable RI variation was $5.1 \times 10^{-4}$. Tian et al. [11] also created an in-line Mach-Zehnder interferometer by concatenating two microfibers together. This design utilizes one abrupt taper to couple light from the core into the cladding, and a second abrupt taper which couples the cladding-mode back to the core. The pairs of microfibers were of $40 \mu \mathrm{m}$ diameter and $707 \mu \mathrm{m}$ in lengths respectively. The sensitivity to RI changes was measured to be $17.1 \mathrm{~nm} / \mathrm{RIU}$. An even higher performance of $2210.84 \mathrm{~nm} / \mathrm{RIU}$ was achieved by Yang et al. [12], using the similar operating principles. The difference is that the two abrupt microfiber sections were tapered along the waist of a uniformly tapered fiber. The fabricated fibers were of 30-49 $\mu \mathrm{m}$ taper diameter and $372 \mu \mathrm{m}$ taper length. Another double-taper based interferometric sensor was reported by Chen et al. [13], with microfibers diameters of $58.5 \mu \mathrm{m}$ and $53.7 \mu \mathrm{m}$, and lengths of $2.4 \mathrm{~mm}$ and $2.2 \mathrm{~mm}$ respectively. The RI sensitivity was $4000 \mathrm{~nm} / \mathrm{RIU}$ at the operating wavelength of $1610 \mathrm{~nm}$ and RI around 1.33.

Wang et al. [14] fabricated a structure consisting of a small core fiber sandwiched between two standard single-mode fibers. By utilizing a focused CO2 laser beam, 20 tapers of $90 \mu \mathrm{m}$ diameter are periodically written along the small core fiber. The wavelength response generated by the multimode interference shifts as RI changes, and an average sensitivity of $226.6 \mathrm{~nm} / \mathrm{RIU}$ was achieved in the RI range from $n=$ 1.33 to1.38. A non-adiabatic microfiber modal interferometer with a large taper slope was reported by Ji et al. [15]. In the RI range from 1.3707 to 1.3769 , the average sensitivity for the $4.61 \mu \mathrm{m}$ diameter microfiber was $15532.24 \mathrm{~nm} / \mathrm{RIU}$ and the maximum sensitivity achieved was $18681.82 \mathrm{~nm} / \mathrm{RIU}$. With an OSA's resolution 
of $10 \mathrm{pm}$, the DL for RI is $5.35 \times 10^{-7}$. Generally, the tapered fiber interferometer is also sensitive to temperature. To differentiate RI from temperature effects in a microfiber based MZI with $65 \mu \mathrm{m}$ diameter and $525 \mu \mathrm{m}$ length, Lu et al. [16] utilized both S-band and C/L-band light sources. Sensitivities as high as $26.087 \mathrm{~nm} / \mathrm{RIU}$ and $0.077 \mathrm{~nm} /{ }^{\circ} \mathrm{C}$ were achieved for the interference order of 144 .

\subsubsection{Gratings assisted microfiber sensors}

The combination of FBGs and microfiber was first reported by Liang et al. [17]. A single etched microfiber ( $6 \mu \mathrm{m}$ diameter) with FBG and another microfiber $(3 \mu \mathrm{m})$ with a pair of FBGs forming a Fabry-Perot interferometer was compared. In both cases, the resonant wavelength shift is proportional to the external RI. Due to the narrower resonance spectral shape of the latter configuration, a DL of $1.4 \times 10^{-5} \mathrm{RIU}$ was attained. Zhang et al. [18] have utilized the larger evanescent field of high-order modes to achieve a sensitivity of $102 \mathrm{~nm} / \mathrm{RIU}$ at $n=1.378$ for a $6 \mu \mathrm{m}$ diameter microfiber. They claimed that the RI sensitivity can be further increased by decreasing the microfiber diameter. More recently, Ran et al. [19] inscribed FBGs on microfibers with diameters ranging from $3.3 \mu \mathrm{m}$ to tens of $\mu \mathrm{m}$ with a $193 \mathrm{~nm} \mathrm{ArF}$ excimer laser. They confirmed that thinner microfiber and high-order modes resulted in higher RI sensitivity. The $\mathrm{LP}_{21}$ peak exhibited a sensitivity of $\sim 600 \mathrm{~nm} / \mathrm{RIU}$ at $n$ $=1.42$.

Wang et al. [20] modeled a microfiber where the external RI affects only the central segment of the grating. Due to the stop-band degeneracy and rapid emergence of spectral modes when an effective phase shift occurs, a highly sensitive sensor can 
be realized. A detection limit of $2.5 \times 10^{-6}$ RIU was estimated for this design, which is an order of magnitude better than non-phase-shifted FBGs. FBGs with high index contrast were fabricated by Liu et al. [21], using the focused ion beam milling technique. Periodic $100 \mathrm{~nm}$ deep grooves were etched on the surface of microfibers with diameters less than $2 \mu \mathrm{m}$. Due to the high index contrast of the grating structure, the length of the grating-written microfiber can be decreased to $\sim 500 \mu \mathrm{m}$. The sensitivity was measured to be $660 \mathrm{~nm} / \mathrm{RIU}$. Recently, Ran et al. [22] proposed a solution involving FBGs on rectangular microfibers. The grating has two Bragg peaks due to the high linear birefringence of the microfiber. They exhibit identical temperature sensitivities. As a result, temperature-independent RI sensing can be accomplished by monitoring the wavelength separation between the two peaks.

It is possible to employ a pair of LPGs with a tapered section in between to form an in-line MZI. Ding et al. [23] presented such a design that operates by partially coupling light to and from a cladding-mode, providing interference with the coreguided mode. A KrF excimer laser with a point-by-point grating writing technique was used to fabricate gratings with a period of $414 \mu \mathrm{m}$, in order to excite a claddingmode of the ninth order for high sensitivity. A DL of $\sim 5.8 \times 10^{-6}$ can be achieved assuming the measurement system has a spectral resolution of $1 \mathrm{pm}$. The same design principles were adopted by Cárdenas-Sevilla et al. [24]. Microfibers with different diameters inserted between the long-period grating pair were fabricated and tested. A minimum DL of $\sim 2.3 \times 10^{-4}$ RIU in a RI range from 1.36 to 1.402 was achieved. Allsop et al. [25] provided an alternative design using only single tapered LPG (400 $\mu \mathrm{m}$ period, $5 \mathrm{~cm}$ length) to avoid the common problem of requiring the two LPGs to 
be identical and $3 \mathrm{~dB}$ in strength for maximum fringe visibility. The light in this case is no longer guided by the core-cladding interface but instead it is initially guided between the cladding and the external medium due to the core-mode cut-off condition along the $6 \mathrm{~mm}$ length microfiber. A DL of $1 \times 10^{-4}$ RIU was obtained within a RI range from 1.3 to 1.34 . A classic Mach-Zehnder interferometer assembled using microfibers was modeled by Wu et al. [26]. Due to the large evanescent field of the microfiber, a small change in the external RI can result in a large phase shift of the guided mode. Simulations using a microfiber of $750 \mathrm{~nm}$ diameter and $1 \mathrm{~mm}$ length foresee the capability of detecting RI variations in the order of $10^{-6}$.

\subsubsection{Surface Plasmon Resonance based microfiber sensors}

One of the first metal-clad microfibers for RI sensing based on the concept of exciting surface plasmon modes was introduced by Díez et al. [27]. A thin layer of gold was evaporated on a MNF of $30 \mu \mathrm{m}$ diameter and $40 \mathrm{~mm}$ length, with the different surface plasmon modes excited by the fiber modes. A sharp optical loss occurs when the propagation constant of the guided mode matches with that of a surface plasmon mode. Hence, surface plasmon waves are strongly dependent on the RI of the surrounding medium. Bueno et al. [28] have shown that by adding a dielectric layer to form an asymmetric double-layer (i.e. $60 \mathrm{~nm}$ thick titanium oxide on $8 \mathrm{~nm}$ thick aluminium), the response of the device can be tuned to obtain surface plasmon mode resonances at shorter wavelengths and at a lower RI range. MonzónHernández et al. [29] had studied different hybrid surface plasmon modes that are 
excited and supported by a semi-cylindrical metallic shell ( $25 \mathrm{~nm}$ thick gold layer) around the microfiber ( $27.5 \mu \mathrm{m}$ diameter, $4 \mathrm{~mm}$ length). The transmission spectrum of the device exhibits multiple resonance peaks, three of which are simultaneously monitored. The measured sensitivity was remarkably high at $127500 \mathrm{~nm} / \mathrm{RIU}$ for RI between $n=1.44-1.454$. A detection limit of $7 \times 10^{-7}$ RIU can be obtained, assuming a wavelength resolution of $10 \mathrm{pm}$. The effect of gold nanoparticles coated on a $1 \mu \mathrm{m}$

microfiber was investigated by Cui et al. [30]. It was found that the resonant wavelength of the surface plasmon was determined by the size of the gold nanoparticles. By tracking the induced wavelength shift, an average sensitivity of $218.67 \mathrm{~nm} / \mathrm{RIU}$ was achieved for RI ranging from $n=1-1.362$ with particle sizes between $20 \mathrm{~nm}$ and $100 \mathrm{~nm}$. Liu et al. [31] showed that the sensitivity of a bare microfiber ( $2.8 \mu \mathrm{m}$ diameter, $14 \mathrm{~mm}$ length) based RI sensor can be enhanced by a factor of 100 using silica nano-spheres (400 $\mathrm{nm}$ diameter) as a modified surface to support Mie-scattering. A tapered fiber localized surface plasmon resonance sensor was demonstrated by Lin et al. [32] for RI sensing and label-free bio-chemical detection. The sensing mechanism is based on the evanescent field absorption of immobilized gold nanoparticles on the tapered fiber surface. A DL of $3.2 \times 10^{-5} \mathrm{RIU}$ was reported for a microfiber of $48 \mu \mathrm{m}$ diameter and $1.25 \mathrm{~mm}$ length with gold nanoparticles of $24 \mathrm{~nm}$ in average diameter.

\subsubsection{PCF microfiber sensors}

It was investigated that miniaturized refractometric sensors based on tapered photonic crystal fibers (PCF) show greater sensitivity compared to normal PCF sensor. Qiu et al. [33] 
investigated, both theoretically and experimentally, the RI sensing properties of PCFs with different diameters, achieved by acid etching instead of tapering. The maximum sensitivity obtained was $750 \mathrm{~nm} / \mathrm{RIU}, 5$ times higher than that of the un-etched version. An enhancement factor of up to 100 times can be expected by optimizing the etching process to achieve thinner and more uniform PCF microfibers. A similar design was proposed and demonstrated by Liu et al. [34], which also used acid-etching to "taper" a section of PCF spliced between two single-mode fibers. The measured sensitivity of a $60 \mu \mathrm{m}$ diameter PCF microfiber was an order of magnitude higher than that of the un-tapered version, at 500 $\mathrm{nm} /$ RIU. Li et al. [35] fabricated a tapered PCF modal interferometer of $30 \mu \mathrm{m}$ diameter and $2 \mathrm{~mm}$ length. The miniature PCF exhibited a sensitivity of more than $1600 \mathrm{~nm} / \mathrm{RIU}$., which is nearly 8 times as high as that of an un-tapered PCF interferometer.

\subsubsection{Other types of microfiber sensors}

Recently, a high sensitivity RI sensor based on a tapered multimode fiber, rather than the conventional single-mode fiber, was demonstrated by Wang et al. [36]. The microfiber diameter and length were $30 \mu \mathrm{m}$ and $675 \mu \mathrm{m}$ respectively. The device was able to achieve a sensitivity of at least $1900 \mathrm{~nm} / \mathrm{RIU}$ and detection limit of $5.23 \times 10^{-6} \mathrm{RIU}$ within the RI range from 1.33 to 1.44 . Biazoli et al. [37] also tapered down a multimode fiber to $55 \mu \mathrm{m}$ spliced between two sections of single-mode fiber to produce a sensitivity of $2946 \mathrm{~nm} / \mathrm{RIU}$ in the RI range from 1.42 to 1.43 [38].

\subsection{Summary of dissertation}

Generally, microfiber can be categorized into two types, namely adiabatic microfiber (type 1) and non-adiabatic microfiber (type 2). The term adiabatic means that the fiber is 
tapered gradually such that the insertion loss into the tapered region is negligible. In this work, both types of the microfibers were studied and demonstrated.

For adiabatic microfiber sensor, there is no higher-order mode excited so that the wavelength response of such microfiber is as flat as before it was tapered. Therefore, it needs to incorporate other techniques into the microfiber to generate a resonant wavelength so that the spectral shift due to external refractive index variation can be monitored. The author has studied such adiabatic microfiber sensors with gratings inscribed. The gratings investigated includes fiber Bragg gratings (FBG) and long period gratings (LPG). Both these two grating techniques were written into the taper waist portion using a $\mathrm{KrF}$ eximer laser. A peak was observed in the reflection spectrum of microfiber Bragg gratings (MFBG) while a dip was observed in the transmission spectrum for the microfiber long period gratings (MLPG). The resonant peak and dip shift when the external refractive index changes. The RI sensitivity of MFBG was found to be moderate, however the sensitivity of MLPG was much higher than normal LPG RI sensors.

MLPG was inscribed in a photosensitive specialty fiber with a W-shaped refractive index profile for sensing of refractive index. The spectrum slightly differs from conventional LPGs. The resonant wavelength shifts towards longer wavelength when external refractive index increases. The sensor achieves a remarkable detection limit of $1.67 \times 10^{-5}$ for refractive index sensing. Although the fiber is tapered into a much smaller diameter, the change in taper diameter is gradual enough to ensure the robustness of the fiber in terms of the mechanical strength. 
For non-adiabatic microfiber sensor, an RI sensor is fabricated by tapering a single-mode fiber into a few micrometers with abrupt taper transitions. The microfiber sensor has shown extraordinary sensitivity and the maximum achieved is $18681.82 \mathrm{~nm} / \mathrm{RIU}$ which is the highest compared with those fiber sensors without extra coating of chemical layers. The high sensitivity in the RI ranging from 1.3337 to 1.37 could be useful in detecting contamination of water, monitoring leakage for oil companies and so on. Moreover, the fabrication process is simple and the sensor is generally compact and robust.

For most of these interferometer-based sensors, there is one common drawback in terms of the measuring range of the refractive index. As the wavelength responses of these modal interferometers appear periodic, as Fig. 1.1 illustrates, once the wavelength shift due to the change of external refractive index exceeds the free spectral range (FSR), it is impossible to identify how much the peak wavelength has shifted. Hence the sensitivity of such sensors cannot be determined [39].

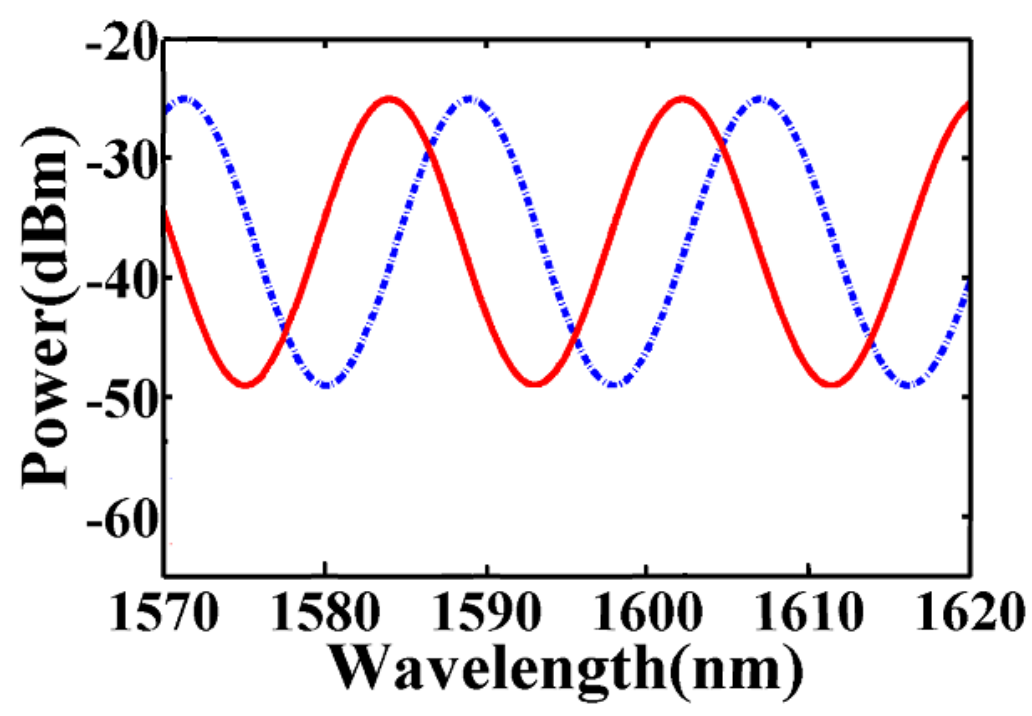

Figure 1.1 Periodic wavelength response of interferometric sensor 
To address this issue, one apparent way is to extend the free spectral range of the interference pattern. Non-adiabatically tapered microfiber refractive index sensor with ultrashort waist length was then investigated. Free spectral range (FSR) is one common issue that needs to be addressed in the interferometry based sensors. Such sensors will not work properly if the resonant wavelengths shift beyond the free spectral range. In this work, the author found that the temperature and the pulling speed of the tapering system are the two crucial parameters that need to be properly controlled to achieve a desired tapering profile. By setting a cooler tapering temperature and fast pulling speed, the author has successfully fabricated ultra-short microfibers with waist length of only $2.4 \mathrm{~mm}$ and total length of 5.5 $\mathrm{mm}$. The resulting FSR is around $80 \mathrm{~nm}$ and the maximum sensitivity achieved for such short microfiber is $25,667 \mathrm{~nm} / \mathrm{RIU}$. Such good performance of the sensor, in terms of measuring range and sensitivity, could potentially be very useful in many practical applications. The author has also studied one of the environmental applications - heavy metal ions detection in water samples.

Heavy metal pollution in natural water environment is always a concern in both human and ecosystem health. The current systems available in the market for detecting heavy metal ions all have common drawbacks such as bulky size, expensive set-up and costly maintenance. This report presents an optical microfiber sensor that is functionalized with chelating agent to detect the existence of specific heavy metal ions of low concentration. To the best of the author's knowledge, the coating of chelating agent in silica microfiber of such small diameter $(3.9 \mu \mathrm{m})$ is novel. A clear spectral shift was observed when the sensor was immersed into metal 
ion solutions including Lead (II), Copper (II), Zinc (II) and Cadmium (II), whose concentrations are in the range of $10-100 \mathrm{ppb}$.

\subsection{Outline of dissertation}

This section gives an outline of the content of this thesis. Chapter 2 outlines the fundamental concept of the optical microfiber including the optical wave guiding principle, optical confinement and evanescent field. Furthermore, a theoretical analysis of adiabaticity which is one of the most important properties of microfiber was conducted. According to the adiabaticity criteria, microfiber can be categorized into two types, namely adiabatic and non-adiabatic microfiber. Finally, this chapter also presented an overview of the fabrication techniques of microfibers and explain the detailed technique used in this work.

Chapter 3 introduces the adiabatically tapered microfiber sensors. For this type of microfiber, the fundamental mode is still the dominant mode and the insertion loss could be negligible. The output spectrum of an adiabatic microfiber is nearly the same as the spectrum of the input light pumped into the microfiber. Therefore, other techniques need to be incorporated so that the response of the sensor to its ambient refractive index can be determined. In this part, fiber Bragg gratings and long period gratings inscribed microfiber sensors were investigated for refractive index sensing.

From chapter 4 onwards, the author mainly focuses on another type of microfiber sensor which is the non-adiabatically tapered microfiber. In chapter 4, a numerical simulation was conducted according to the theoretical analysis in chapter 2. Preliminary results were 
obtained, followed by further exploration of the detection limit of such sensor. Finally, dual parameter sensing of strain and refractive index by connecting a FBG into the microfiber was also studied.

The drawbacks of type 2 microfiber sensor were discussed in chapter 5. The sinusoidal response of the abrupt taper will sometimes cause reading errors if the free spectral range (FSR) is too narrow. In this chapter, the author presents the method to extend the FSR without compromising the sensitivity too much. The author studied the relationship between the taper profile with the tapering temperature and strain applied during the tapering process.

Having optimized the type 2 microfiber sensor to achieve sufficient sensitivity and FSR, the author used the fabricated sensor for RI sensing applications. In Chapter 6, the author describes a potential application in environmental water quality monitoring. While water contamination in general, is a major environmental issue, there are some contaminants that are more critical to our health. Specifically, for the purpose of this thesis, we looked into the issue of heavy metal concentration in water. To do so, the author coated metal chelating agents on the fiber surface to trap the heavy metal ions that may be present in the water samples, onto the surface of the fiber. A detailed process of surface functionalization will be described in this chapter, and the surface was examined by scanning electron microscope (SEM) before and after coating, to confirm that the chemical process is successfully.

Since all fiber sensors are sensitive to temperature and strain variations, their effect on our sensor need to be properly studied and minimized. In chapter 7, the author will describe a testing system that was designed to minimize issues arising from strain and temperature for the non-adiabatic microfiber sensor used for water quality monitoring. Subsequently, 
final characterization of metal ion detection using the surface functionalized sensor was conducted.

The last portion of this thesis is a summary of the research work done, along with recommendations for future work which encompasses the engineering work on the miniaturization and stabilization of the entire detection system. 


\section{Chapter 2 : Introduction of microfiber}

\subsection{General introduction}

Optical microfibers are essentially optical fibers that are tapered to diameters of a few micrometers and the core diameter is comparable to the wavelength of the light propagating through it. Microfibers are usually fabricated by heating and stretching normal-sized optical fibers. The result is a biconical taper that provides a smooth lossless connection to other fiberized components. By controlling the pulling rate during the fabrication process, the taper profile can be fine-tuned to suit the application [40]. Optical materials other than silica have been used to fabricate microfibers, including phosphate [41], tellurite [41], lead silicate [42], bismuthate [42] and chalcogenide glasses [43] and a variety of polymers [44-47]. The remarkable optical and mechanical properties exhibited by microfibers are constantly exploited for optical sensing and include large evanescent fields, strong optical confinement, flexibility, configurability and robustness. Such desirable characteristics have gathered much attention in recent years and it is what makes microfibers an excellent choice for optical sensors [38].

\subsection{Optical waveguiding properties of microfiber}

Light launched into a microfiber will propagate as core modes which are defined to be the state when their effective refractive index lies between the cladding and core indices. The effective refractive index decreases monotonically along the down-taper transition, and the mode becomes guided by the cladding/air interface. To analyze the mode propagation in the microfiber, the exact solution of the Maxwell equations needs to be found [48] since 
the weakly guiding approximation is not valid as a result of the large index difference between the cladding and air:

$$
\begin{gathered}
{\left[\frac{J_{v}^{\prime}(U)}{U J_{v}(U)}+\frac{K_{v}^{\prime}(U)}{W K_{v}(U)}\right]\left[\frac{J_{v}^{\prime}(U)}{U J_{v}(U)}+\left(\frac{n_{s u r r}}{n_{M N F}}\right)^{2} \frac{K_{v}^{\prime}(U)}{W K_{v}(U)}\right]=} \\
\boldsymbol{v}^{2}\left(\frac{1}{\boldsymbol{U}^{2}}+\frac{1}{\boldsymbol{W}^{2}}\right)\left(\frac{1}{\boldsymbol{U}^{2}}+\left(\frac{\boldsymbol{n}_{\text {surr }}}{\boldsymbol{n}_{M N F}}\right)^{2} \frac{1}{\boldsymbol{W}^{2}}\right)
\end{gathered}
$$

where $U=r \sqrt{k_{0}^{2} n_{M N F}^{2}-\beta^{2}}, W=r \sqrt{\beta^{2}-k_{0}^{2} n_{\text {surr }}^{2}}, J_{v}$ is the $v^{\text {th }}$ order Bessel function of the first kind, $K_{v}$ is the $v^{\text {th }}$ order modified Bessel function of the second kind, $n_{M N F}$ and $n_{\text {surr }}$ are the refractive indices of the microfiber $(\sim 1.45)$ and the surrounding medium. $\beta$ is the effective propagation constant of the optical mode. $r$ is used to denote the core radius and correspond to the center from the core-cladding interface in solid fibers and to the cladding-air interface in microfibers. The propagating constant of hybrid modes can be calculated by solving Eqn. (2.1). The V number can be expressed as:

$$
V=\sqrt{U^{2}+W^{2}}=\frac{2 \pi}{\lambda} * r * \mathrm{NA}
$$

As it is in optical fibers, the threshold for microfibers experience single-mode operation when $\mathrm{V}<2.405[38,49]$.

\subsection{Optical confinement}

Microfibers can confine light to the diffraction limit for lengths that are thousands of times longer than the typical Rayleigh length. In free space Gaussian beams, the Rayleigh criterion limits the maximum confinement length to a few microns, however in optical 
microfibers, confinement lengths can be much longer that are only limited by microfiber propagation loss. As shown in Figure 2.1, when the optical fiber diameter decreases, the V number and spot size $\omega$ initially decreases until the point of maximum confinement within the core. After that, the mode is no longer guided in the core and $\omega$ suddenly increases to a maximum associated with cladding guiding. The physical meaning of the spot size $\omega$ is the mode distribution area in the cross-sectional view of the fiber and the diameter of such area is called the mode field diameter (MFD). The common way to determine the MFD is to measure the far field profile, i.e., the beam profile outside the fiber in a distance which is much larger than the Rayleigh length. This can be done with a camera mounted in certain distance to the fiber end, or by scanning the far field with a small photodetector.

For even smaller diameters, $\omega$ decreases with decreasing $\mathrm{V}$ until it reaches a minimum of around $\mathrm{V} \sim 2$, which is typical of microfibers. In this condition, the mode is only weakly guided by the waveguide, and hence $\omega$ can be orders of magnitude larger than the physical diameter of the microfiber and a larger fraction of power resides in the evanescent field. For $\mathrm{V}<0.6$, the beam spot size can continue to expand until it becomes orders of magnitude larger than $r$ [38] as shown in Figure 2.1. 


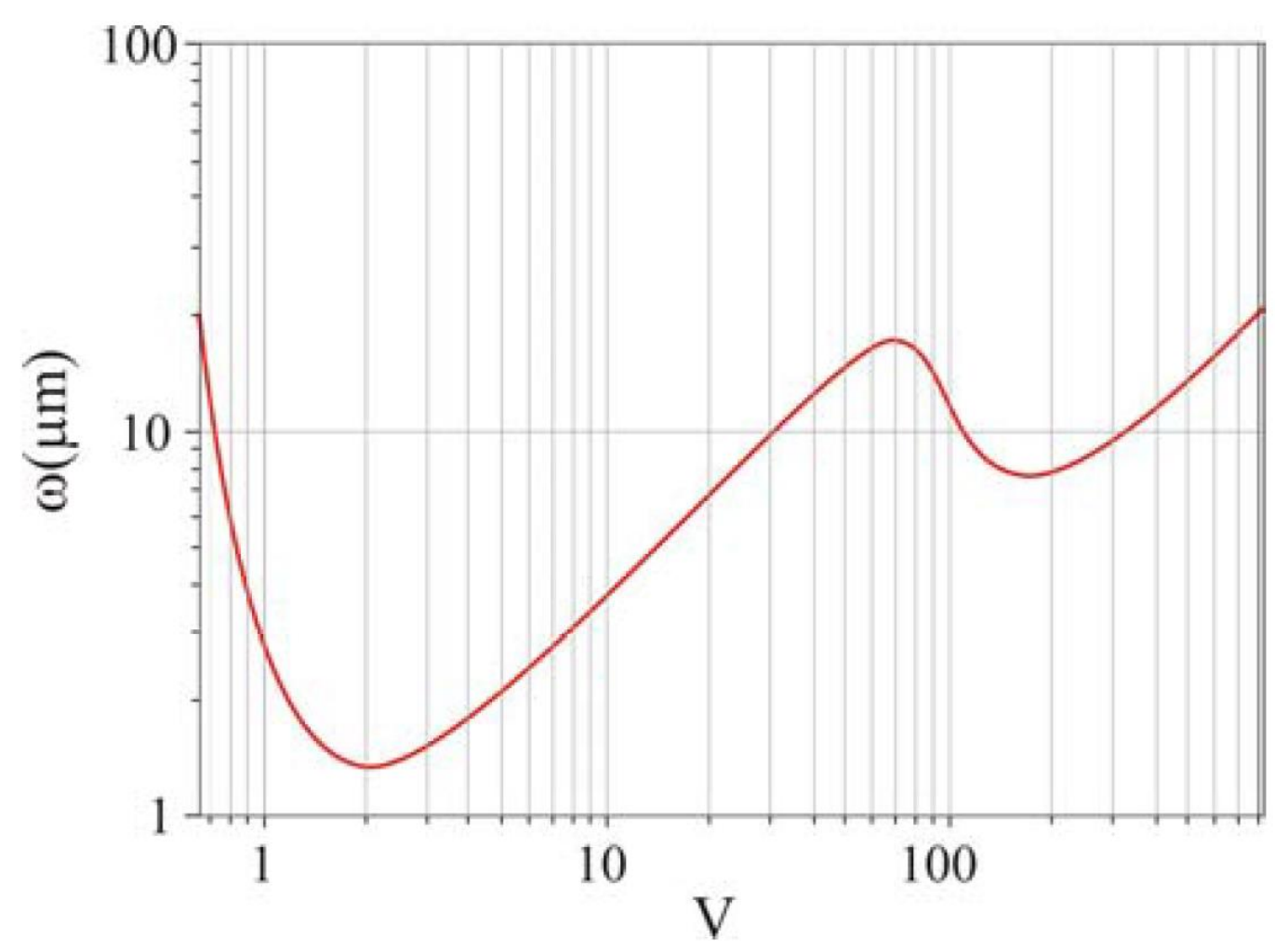

Figure 2.1 Dependence of the spot size on the V number of a microfiber. The two troughs represent the points of maximum confinement in the cladding/core. [38]

\subsection{Evanescent field}

When $\mathrm{V}<<1$, a large portion of the power propagates as evanescent field outside the microfiber or nanofiber (MNF). The extension of the evanescent field and the fraction of power $\eta_{E F}$ propagating in it depend on the ratio $\lambda / r$ and can be obtained from the component of the Poynting vector along the direction of the beam propagation $S_{z}$ [49].

$$
\eta_{E F}=\frac{\int_{o u t} S_{Z} d A}{\int_{\text {in }} S_{Z} d A+\int_{o u t} S_{z} d A}=\frac{\int_{r}^{\infty} S_{z} d A}{\int_{0}^{r} S_{Z} d A+\int_{r}^{\infty} S_{Z} d A}
$$


where $\int_{i n} S_{Z} d A$ and $\int_{o u t} S_{Z} d A$ are the integrals of $S_{Z}$ over the MNF cross section and outside the MNF respectively. Figure 2.2 shows the dependence of $\eta_{E F}$ on the normalized wavelength $\lambda / r$ for silica MNFs. When the surrounding medium is air, $\eta_{E F}$ reaches 0.5 at $\lambda / r \sim 4$, meaning that half of the power is propagating outside the MNF when the MNF radius is a quarter of the wavelength of light propagating in it. $\eta_{E F}$ increases for increasing surrounding refractive indices when the normalized wavelength $\lambda / r$ is kept constant. For example, in a medium with surrounding refractive index of $n_{\text {surr }}=1.373$, more than $90 \%$ power lies in the evanescent field when $\lambda / r \sim 4$. Therefore, to enhance the evanescent field, polymers or Teflon can be used to embed the MNF. To improve the $\eta_{E F}$ even further, hollow MNFs have also been proposed [50]. Large evanescent field is particularly important in sensors and resonators where a significant fraction of the propagating power needs to interact with surrounding medium [38]. 


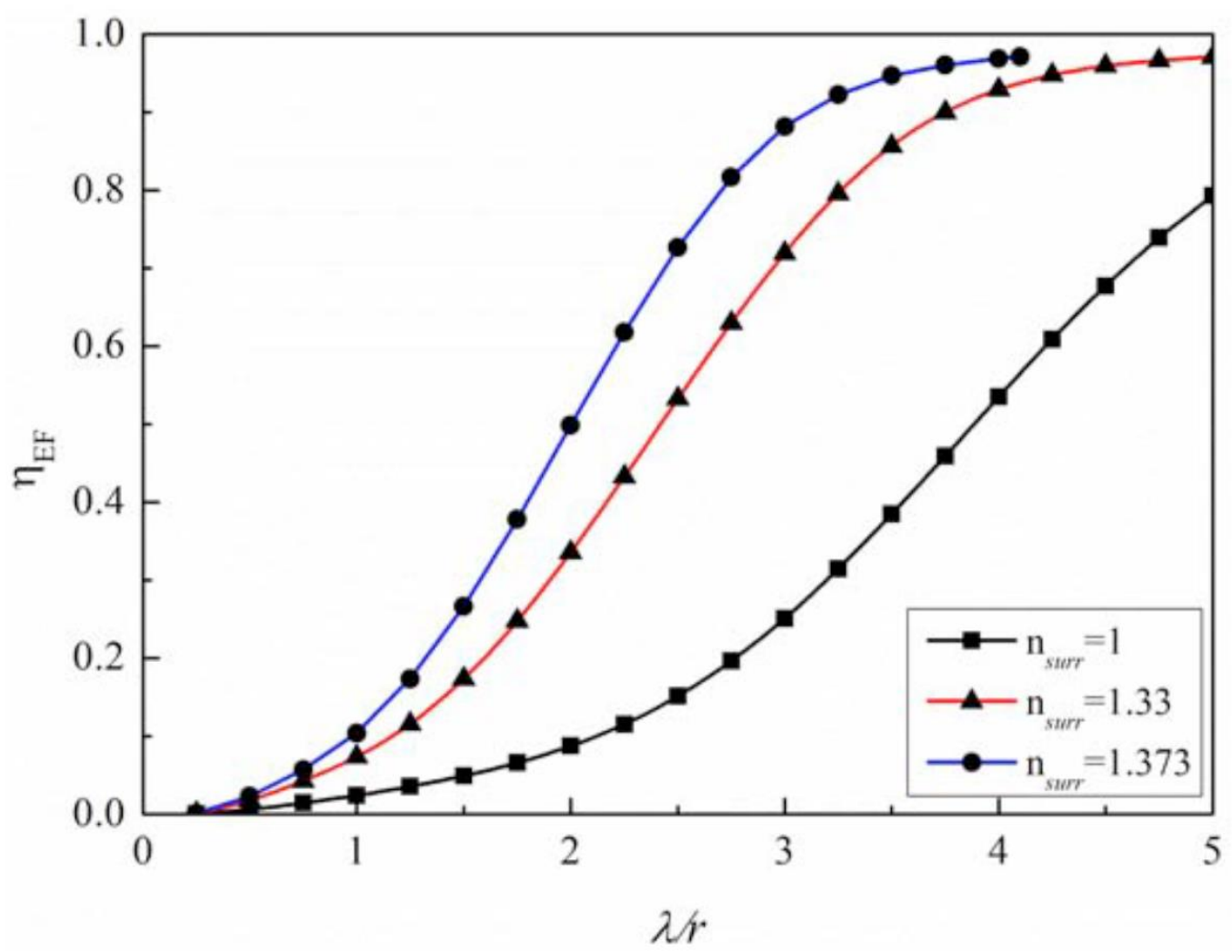

Figure 2.2 Dependence of the power fraction propagating in the evanescent field $\boldsymbol{\eta}_{\boldsymbol{E} F}$ on the normalized wavelength $\lambda / \mathrm{r}$ in different refractive index surroundings [38].

\subsection{Adiabaticity of fiber taper}

Microfiber is fabricated by tapering an optical fiber to diameter as small as a few micrometers. The taper is called adiabatic when the fiber is tapered gradually and exhibits negligible transmission loss. J. D. Love et al [51] have studied the single-mode fiber taper's adiabaticity based on two criteria. 


\subsubsection{Length-scale criterion}

The first criterion is derived from the physical argument that taper length-scale must be much larger than the coupling length between the fundamental mode and the dominant coupling mode for power loss to be small [52]. As illustrated in Figure 2.3, the taper angle is defined as

$$
\Omega(z)=\tan ^{-1}\left(\frac{d \rho(z)}{d z}\right)
$$

where $\rho(\mathrm{z})$ is the radius along the $\mathrm{z}$ axis. In this criterion, the taper angle is assumed to be sufficiently small so that

$$
\mathrm{z}_{t} \approx \frac{\rho}{\Omega}
$$

Assuming the taper to be axisymmetric, as is usually the case with a single fiber taper, then the fundamental $\mathrm{LP}_{01}$ mode can couple only to the modes with the same azimuthal symmetry, i.e. to the higher order $\mathrm{LP}_{0 m}$ cladding modes. If we are aiming to minimize loss from the fundamental mode, it is obvious that coupling will be mainly to the higher-order mode with propagation constant that is closest to the fundamental mode, i.e. the $\mathrm{LP}_{02}$ mode. The coupling length between the two modes is taken to be the beat length between the fundamental mode and higher-order mode, and takes the form

$$
\mathrm{z}_{b}(z)=\frac{2 \pi}{\beta_{1}(z)-\beta_{2}(z)}
$$


where $\beta_{1}(z)$ and $\beta_{2}(z)$ are the propagation constants of the two modes.

When $z_{t} \gg z_{b}(z)$ everywhere along the taper, the coupling loss will be negligible and the fundamental mode will propagate nearly adiabatically. Whereas significant coupling will occur if $z_{t} \ll z_{b}(z)$. This indicates that $z_{t}=z_{b}(z)$ is a condition just in between adiabatic and lossy tapers. Therefore, the taper angle can be derived as

$$
\Omega(z)=\frac{\rho(z)\left(\beta_{1}(z)-\beta_{2}(z)\right)}{2 \pi}
$$

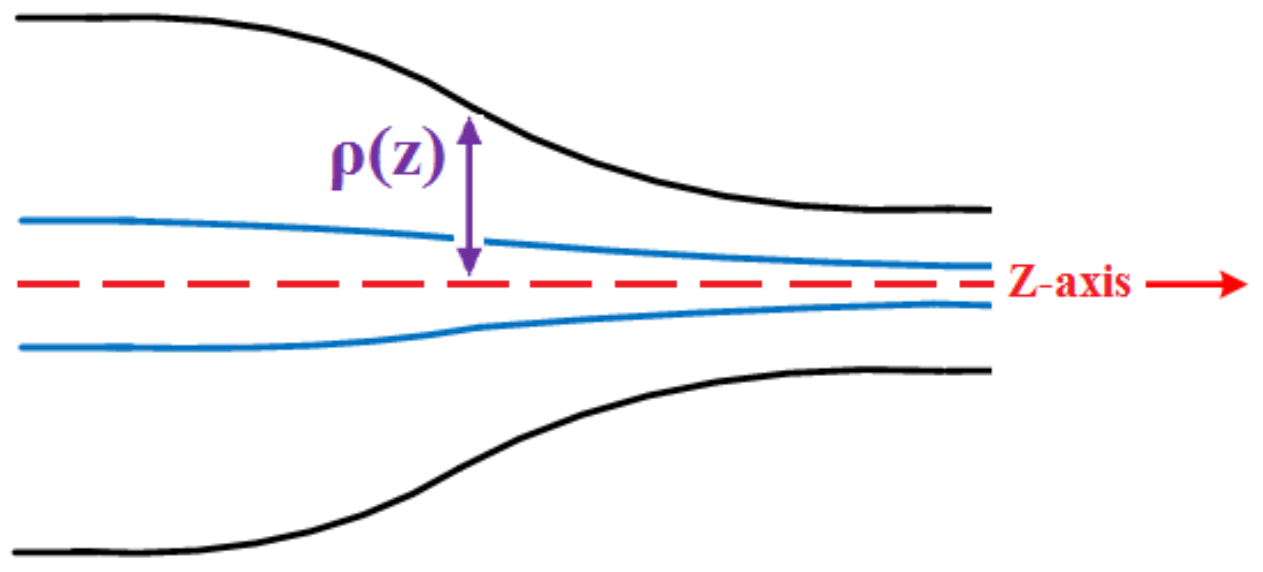

Figure 2.3 Schematic view of a taper profile

\subsubsection{Weak power transfer criterion}

The loss from the fundamental mode due to tapering can be calculated by expressing the field along the taper as a superposition of the fundamental and cladding modes. By solving a set of coupled mode equations which manipulates the amplitudes of the modes, the loss 
can be quantified [53-55]. If we still assume that coupling only occur between $\mathrm{LP}_{01}$ and $\mathrm{LP}_{02}$, the amplitudes of these two modes can be well approximated by [55]

$$
\begin{gathered}
a_{1}(z)=a_{1}(0) \exp \left\{i \int_{0}^{z} \beta_{1}\left(z^{\prime}\right) d z^{\prime}\right\} \\
a_{2}(z)=a_{1}(0) \exp \left\{i \int_{0}^{z} \beta_{2}\left(z^{\prime}\right) d z^{\prime}\right\} \int_{0}^{z} C\left(z^{\prime}\right) \times \exp \left\{i \int_{0}^{z^{\prime}}\left(\beta_{1}\left(z^{\prime \prime}\right)-\beta_{2}\left(z^{\prime \prime}\right)\right) d z^{\prime \prime}\right\} d z^{\prime}
\end{gathered}
$$

where $a_{1}(0)$ is the amplitude of the fundamental mode at the beginning of the taper, and $C$ is the coupling coefficient between the two modes, which is expressed as

$$
C=\frac{1}{2} \frac{k}{\beta_{1}(z)-\beta_{2}(z)} \frac{d \rho(z)}{d z} \frac{1}{n_{c o}} \frac{\int_{A_{\infty}} \psi_{1} \psi_{2} \frac{\partial n^{2}}{\partial \rho(z)} d A}{\sqrt{\int_{A_{\infty}} \psi_{1}^{2} d A \int_{A_{\infty}} \psi_{2}^{2} d A}}
$$

where $n_{\mathrm{co}}$ is the maximum core index, $k$ is the free space wave number, $\lambda$ is the free-space wavelength, $A_{\infty}$ is the finite cross-section and $\psi_{1}, \psi_{2}$ are the scalar wave equation fields for the fundamental and local modes. The power of higher-order mode at taper end, $z=L$, should be as small as

$$
\left|\int_{0}^{L} C\left(z^{\prime}\right) \exp \left\{i \int_{0}^{z^{\prime}}\left(\beta_{1}\left(z^{\prime \prime}\right)-\beta_{2}\left(z^{\prime \prime}\right)\right) d z^{\prime}\right\}\right| \ll 1
$$


Since the taper is gradual so as to ensure the adiabaticity, maximum power transfer between the two modes takes place at half of the beat length. The integration result of the exponential part (phase) is almost constant over the beat length so that the inequality becomes

$$
\frac{C z_{b}}{\pi} \ll 1
$$

It can be assumed that

$$
C \mathrm{z}_{\mathrm{b}}=\mathrm{c}
$$

if we assign a constant $c \ll \pi$. Therefore, the taper angle can be written as

$$
\Omega(z)=\frac{c}{C_{n}} \frac{\rho(z)\left(\beta_{1}(z)-\beta_{2}(z)\right)}{2 \pi}
$$

where $C_{n}$ is the normalized coupling coefficient and can be expressed as

$$
C_{n}=C \frac{\rho}{d \rho / d z}
$$

Both the length-scale criterion and weak power transfer criterion indicate that when the core diameter is reduced to a small amount, in order to maintain low loss taper, the taper angle also needs to be sufficiently small by making the fiber taper to vary gradually. 


\subsection{Fabrication of microfiber}

Typical microfibers are fabricated from amorphous materials such as glass and polymers. For these amorphous materials, the top-down physical drawing has been proven to be very successful for yielding microfibers with circular cross-section, uniform diameter, smooth surface and long length [56]. Glass microfibers are usually fabricated by taper drawing of glass fiber or bulk glass at high temperature. The heating source can be a flame (usually a hydrogen flame) [57-62], an electrical heater [42, 63, 64], or a laser-heated tube [65-70]. Depending on the heating conditions and the material properties around the melting temperature, the drawing speed varies from tens of micrometers to several meters per second. By precisely controlling the drawing speed, heating conditions and subsequently the geometric profile of the tapering region, a microfiber drawn from a standard glass fiber with both ends naturally connected to the original fiber (usually called a biconical microfiber or fiber taper), can exhibit high-efficiency (up to almost 100\%) or so-called adiabatic connection to the optical fiber, making it very convenient for in/out-coupling of light via standard optical fiber system [71]. Furthermore, one can also fabricate microfibers with abrupt down taper and up taper to make it directly an interferometric sensor. 


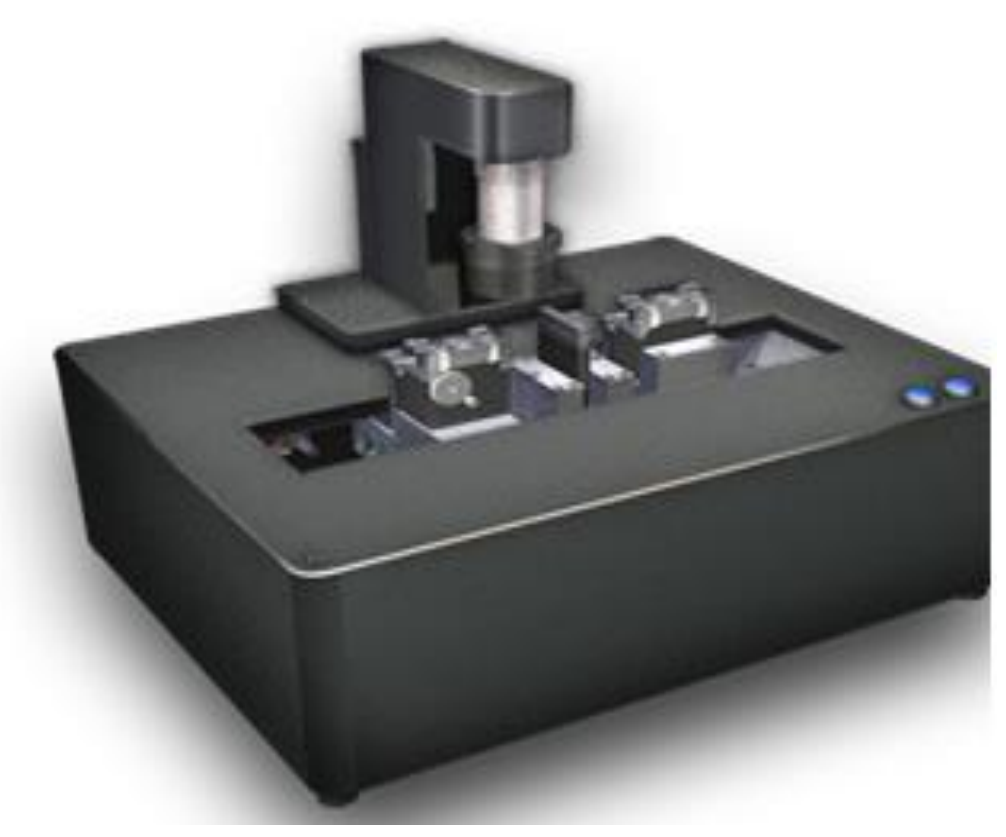

Figure 2.4 Fiber tapering machine

In this work, the author used a commercial fiber tapering machine (Vytran GPX-3000) for microfiber fabrication, as shown in Fig. 2.4. The fiber was placed and held at the two fiber holding blocks (FHB), in between there is a furnace to generate heat to soften the fiber. The machine is controlled by software that can be used to predetermine the machine parameters such as starting power of the filament, the power degradation rate, the pulling speed of the FHB. The tapered fiber consists of three parts, namely the down taper, the waist and the up taper, as illustrated in Fig. 2.5. 


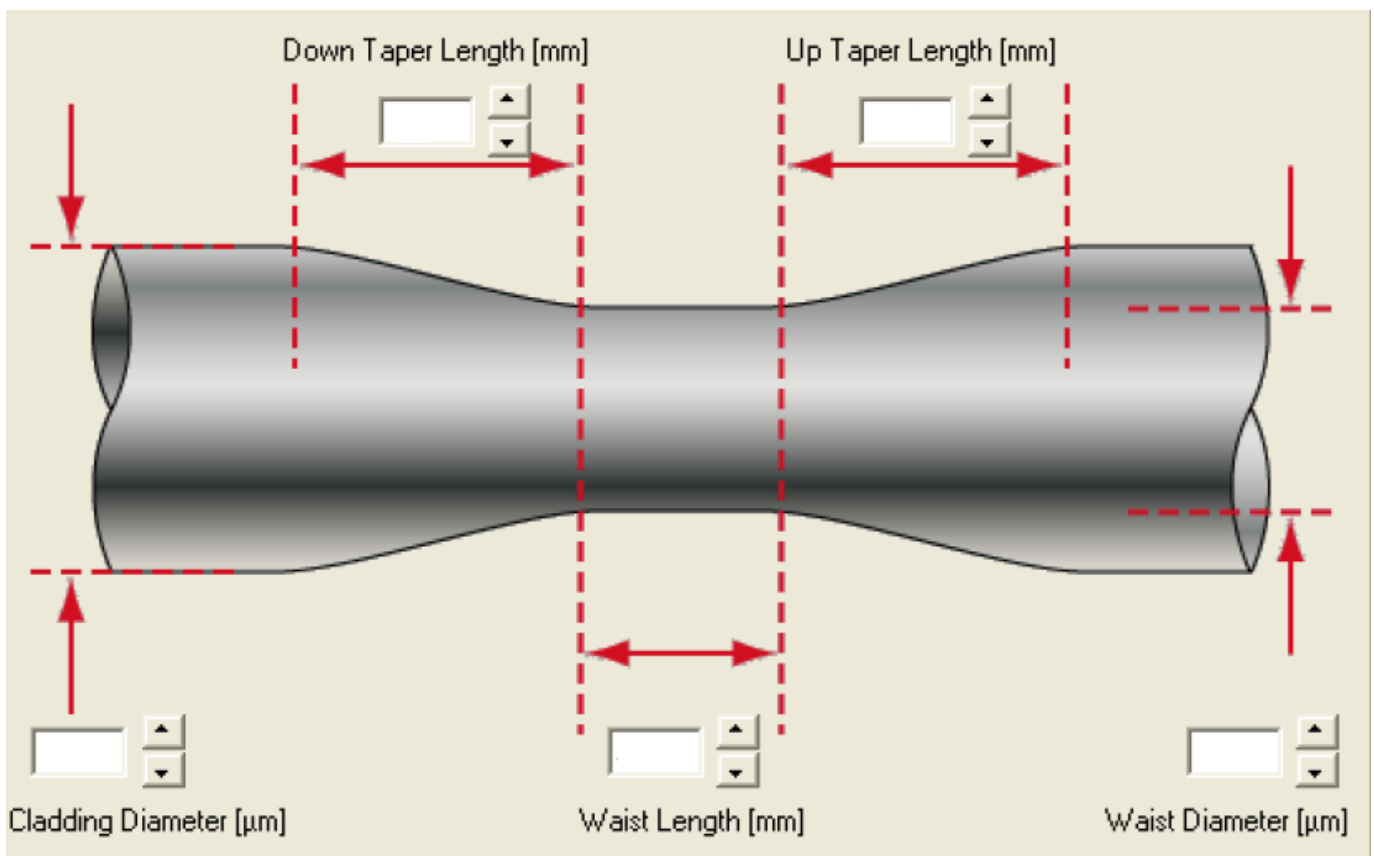

Figure 2.5 Schematic view of a taper profile

The down taper and up taper are also called the taper transitions. Fig. 2.6 shows an example of the fabricated adiabatically tapered microfiber observed under optical microscope. This fiber taper has diameter of around $7.6 \mu \mathrm{m}$ with waist length of $20 \mathrm{~mm}$. The taper transition lengths are around $15 \mathrm{~mm}$. The transmission spectra of the original output of light source and output after tapering are depicted in Fig. 2.8. One can see that the insertion loss of the tapered fiber at any specific wavelength from 1520 to $1620 \mathrm{~nm}$ is smaller than $1 \mathrm{~dB}$ and the inter-modal coupling is nearly negligible since the amplitudes of those spikes (red line) are considerably small. 

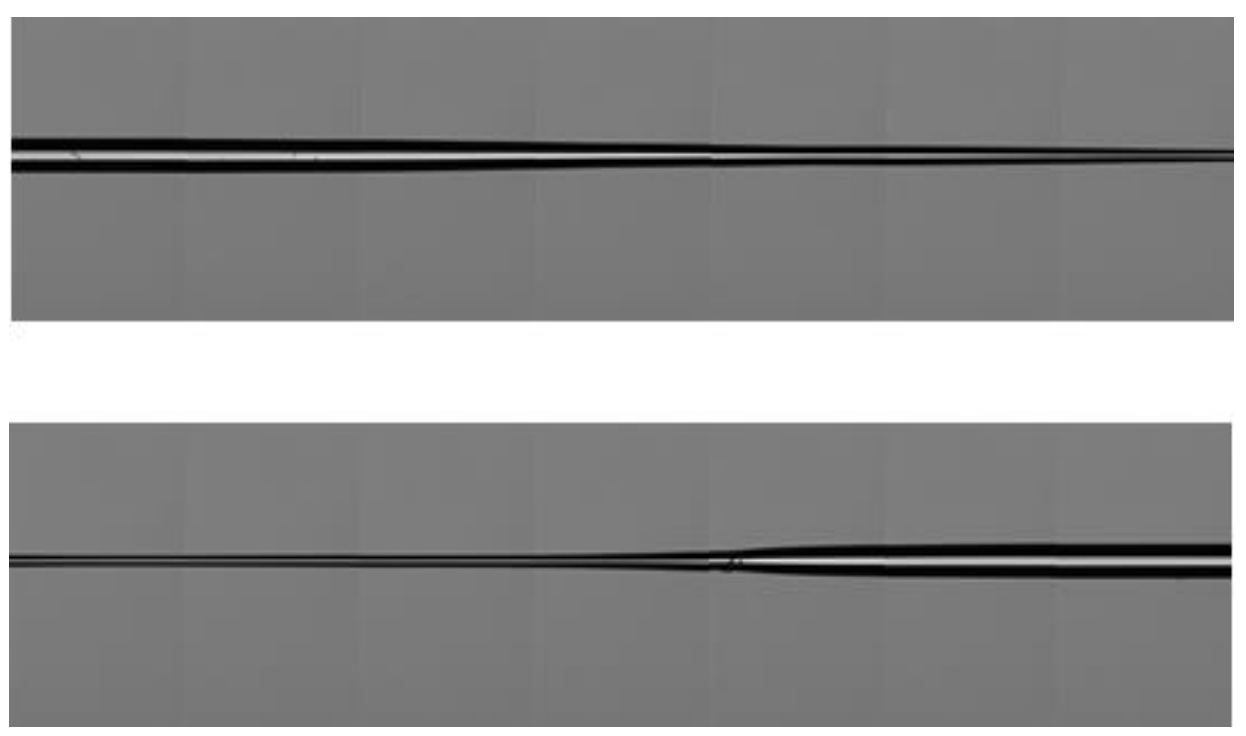

Figure 2.6 Microscopic view of an adiabatically-tapered optical fiber

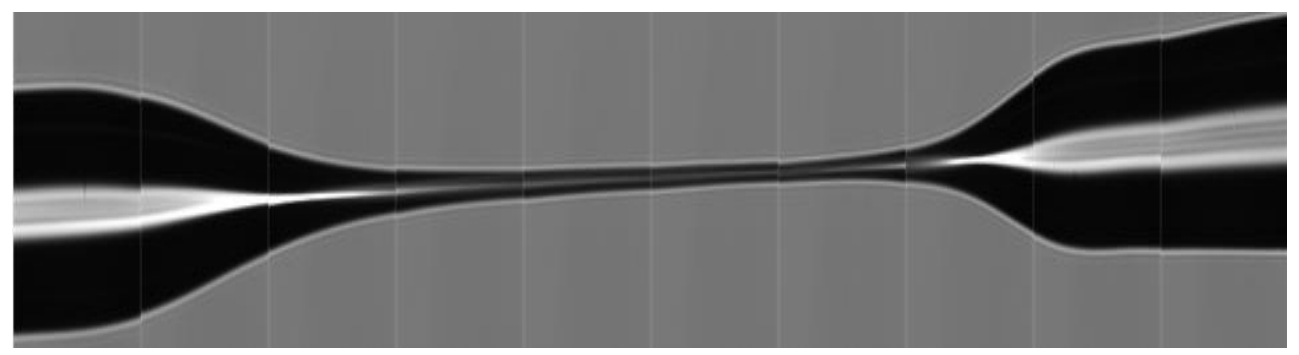

Figure 2.7 Microscopic view of a non-adiabatically-tapered optical fiber

Fig. 2.7 shows an image of a non-adiabatically tapered microfiber. The center waist has a diameter of approximately $8 \mu \mathrm{m}$ and its length is close to $3 \mathrm{~mm}$. The down taper length is approximately $2 \mathrm{~mm}$ and the up taper length is approximately $1.2 \mathrm{~mm}$. The resulting transmission spectrum for this microfiber is illustrated in Fig. 2.9. Higher-order modes are excited and the interference between the dominant higher-order mode and the fundamental mode occurs, resulting in a sinusoidal-like reflected spectrum. 


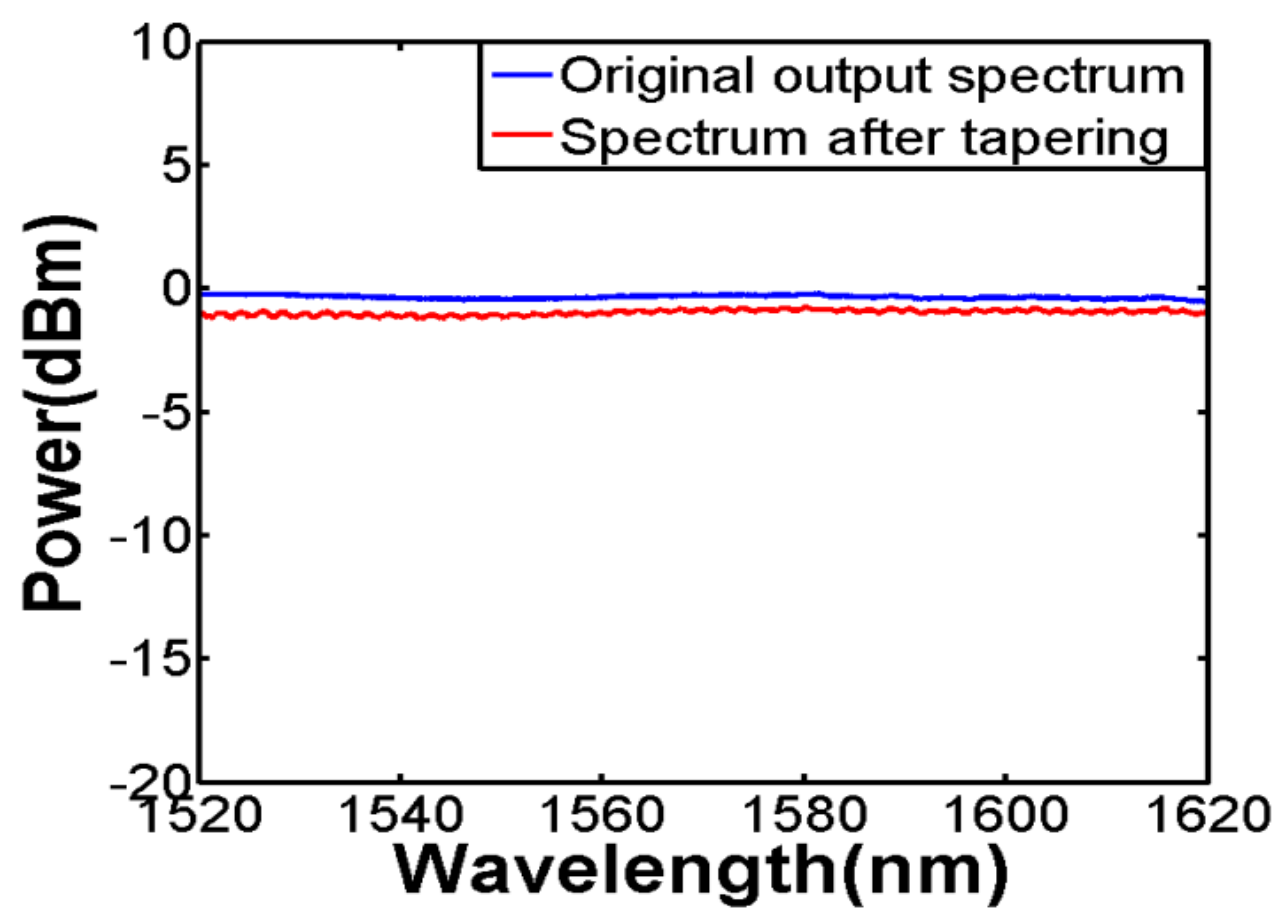

Figure 2.8 Transmission spectra of an adiabatically tapered microfiber

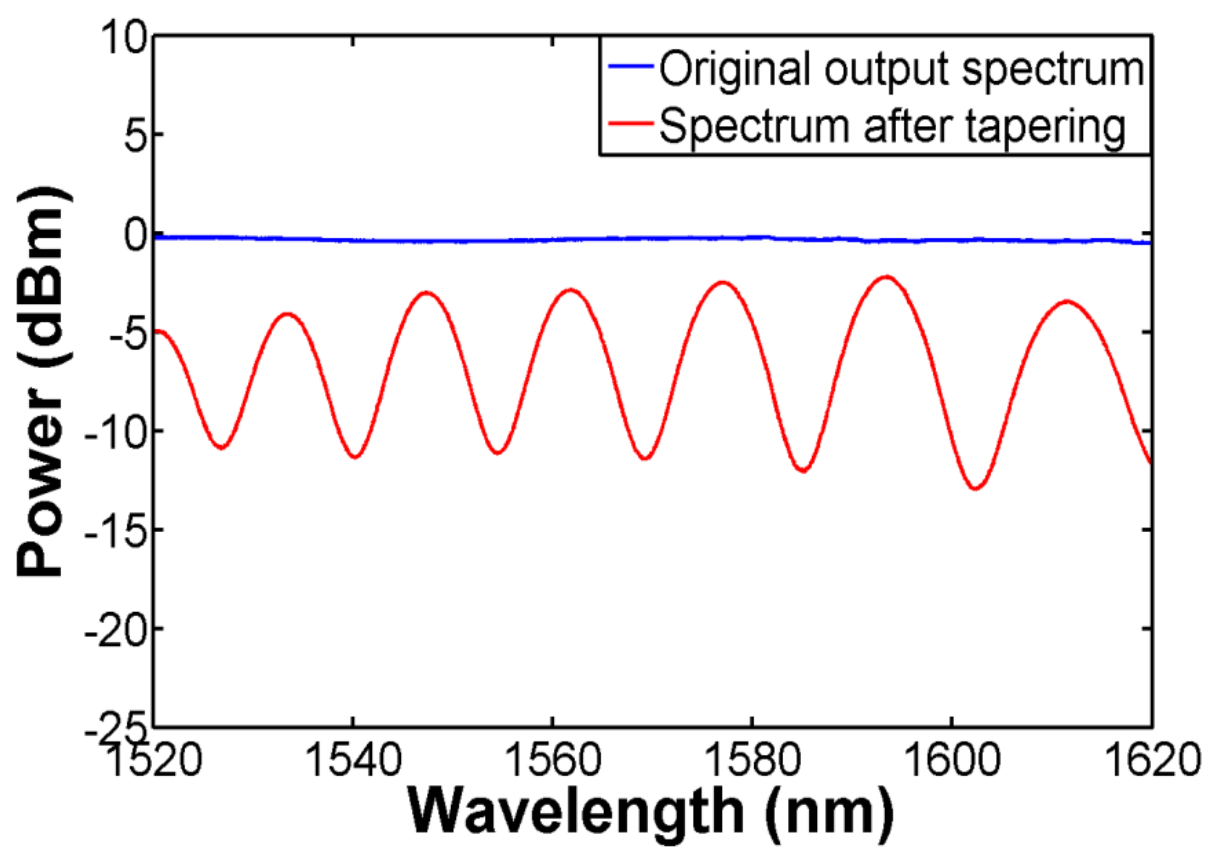

Figure 2.9 Transmission spectra of a non-adiabatically tapered microfiber 
One thing worth noting is that the non-symmetric property of such microfiber taper is quite common in most of the taper drawing systems since the tension to form the down taper and the up taper are different. Before the taper drawing starts, a tension of 20 grams is applied to the fiber since an initial tension is necessary to make the fiber elongate. Once the fiber starts to melt, the two fiber holding stages move towards the same direction but the velocities are varied in order to maintain the tension of the fiber along the taper process. As the taper process progresses, the down taper and the waist parts are formed. The fiber then becomes very fragile such that it cannot even bear a tension of 5 grams which is not sufficient to elongate the up taper length further. That is why the length of down taper will usually be greater than the up taper although the lengths are preset to be the same in the software. 


\section{Chapter 3 : Adiabatically tapered microfiber sensors}

\subsection{Overview}

In a perfect optical fiber, where the refractive indices are homogenous and physical dimensions are uniform along the length of the fiber, the fundamental guided core mode will continue to propagate in the fiber. However, due to the manufacturing process which causes fiber waveguide imperfections, non-uniform refractive index and physical profile variations exist in the fiber system and this causes coupling between the fundamental mode and other modes such as the radiation modes. Another means of introducing perturbation into the fiber core will be by the introduction of periodic refractive index modulation in the fiber core. Two such optical devices with uniform periodic modulation of refractive index induced into the optical fiber core are fiber Bragg gratings and long period gratings. Both gratings have attracted attention from researchers for sensing applications. However, due to weak evanescent field penetration into the medium surrounding the fiber, modifications to the optical fiber may have to be made before inscribing gratings so as to enhance its performance for refractive index sensing.

In this chapter, a general introduction to both fiber Bragg gratings (FBG) and long period gratings (LPG) will be presented, followed by various characterizations of the microfiber FBG and microfiber LPG sensors.

The propagation of light along an optical fiber can be described in terms of a set of guided electromagnetic waves commonly referred to as 'modes' of the optical fiber waveguide. 
Each guided mode is a pattern of electric and magnetic field distributions that is repeated along the fiber at equal intervals. A fundamental guided mode propagating along an optical fiber has its field tightly confined to the core. Although the fundamental mode is concentrated in the core, the electric field of this mode does not go to zero at the corecladding interface as shown in Fig. 3.1.

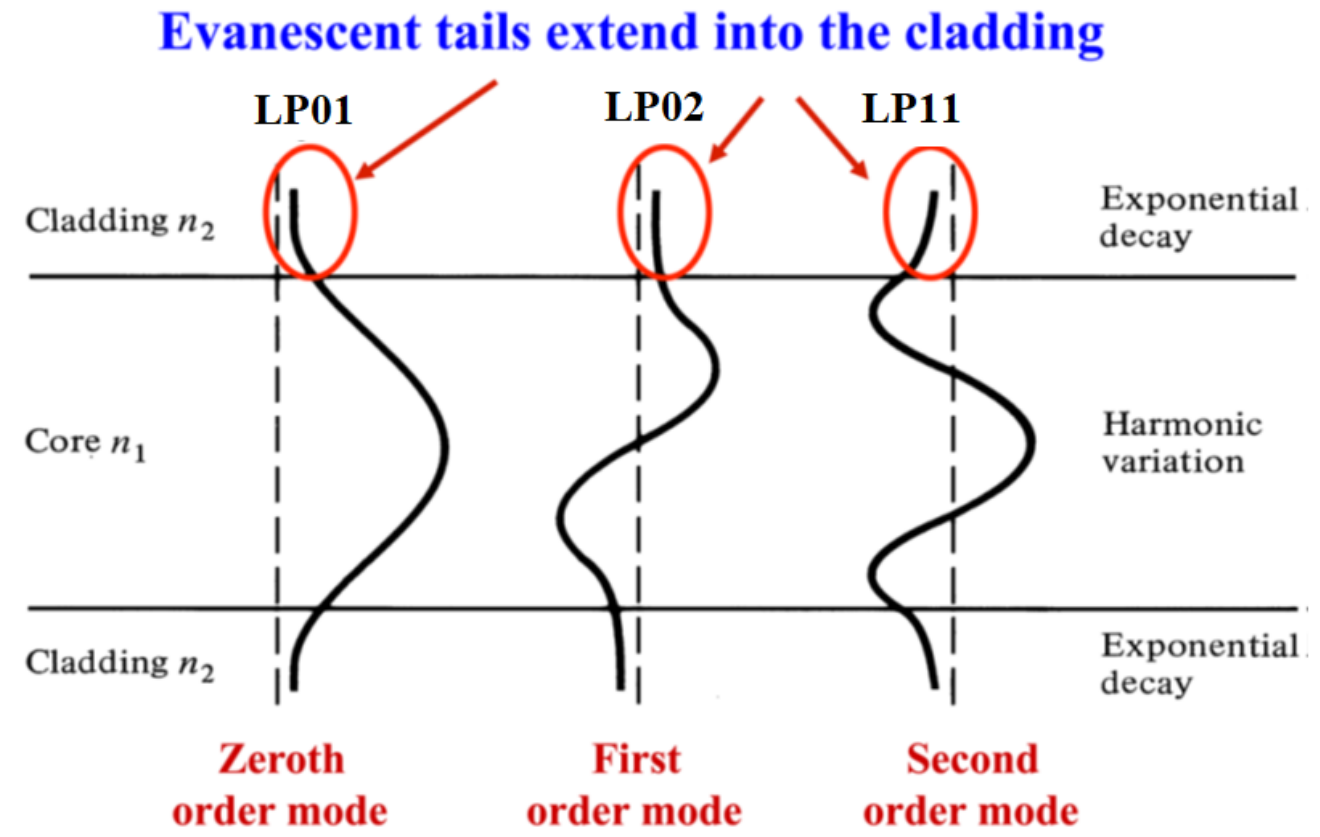

Figure 3.1 Electric field distribution of guided modes in an optical fiber (longitudinal cross-sectional view) [72]. 


\subsection{Microfiber Bragg gratings}

\subsubsection{Introduction of $F B G$}

Fiber Bragg gratings or FBGs, first developed in 1978, were based on phase-matching between the forward propagating and backward propagating $\mathrm{LP}_{01}$ guided core mode in the presence of a periodic modulation of refractive index in the core of the optical fiber [73]. Figure 3.2 is a graphic representation of the backward propagation core mode in an FBG

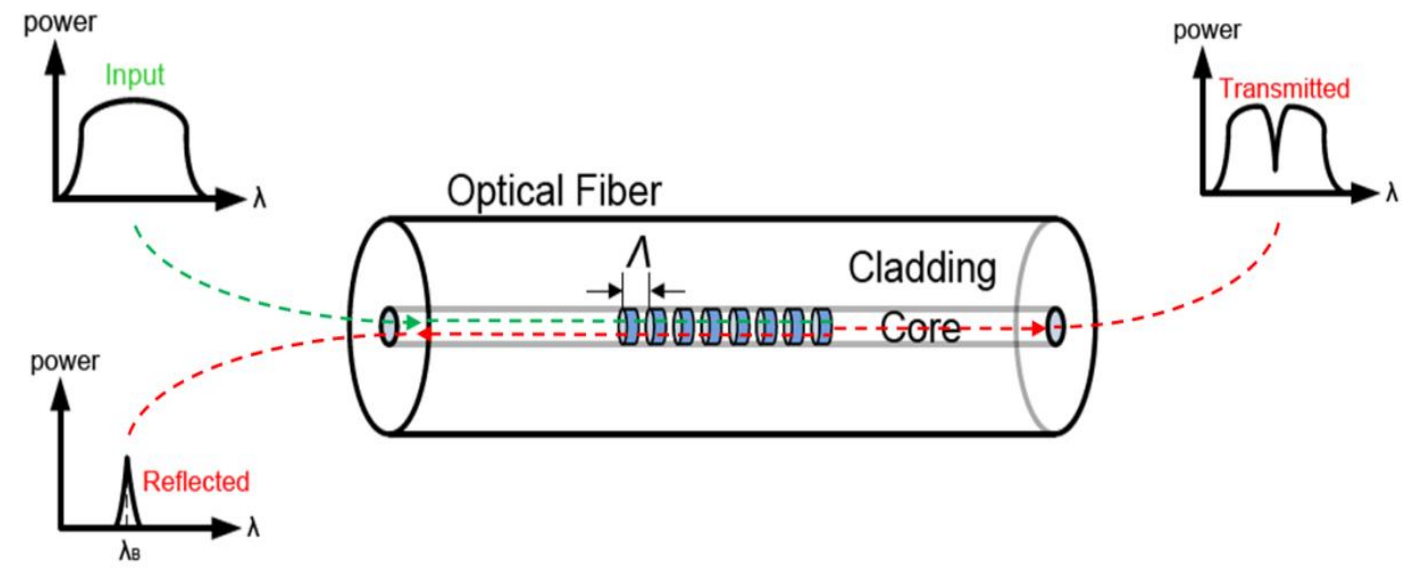

Figure 3.2 Graphic representation of fiber Bragg gratings

The phase mismatch $\Delta$ between the propagation modes can be expressed as [74]

$$
\Delta=\beta_{1}-\beta_{2}-\frac{2 \pi}{\Lambda}
$$


At the phase-matching condition (with $\Delta=0$ ), the propagation constants $\beta_{1}$ and $\beta_{2}$ for the core mode and counter propagating mode will have a difference related to the perturbation $\operatorname{of} \operatorname{period} \Lambda$,

$$
\begin{gathered}
\beta_{1}-\beta_{2}=\frac{2 \pi}{\Lambda} \\
\beta_{1}=n_{\text {core }}^{\text {eff }} k \\
\beta_{2}=-\beta_{1}=-n_{\text {core }}^{\text {eff }} k
\end{gathered}
$$

where $n_{\text {core }}^{\text {eff }}$ is the effective refractive index of the fundamental core mode and $k=\frac{2 \pi}{\lambda}$ is the free-space propagation constant. The negative sign in Eqn. (3.4) indicates propagation in the opposite direction. By substituting Eqn. (3.3) and Eqn. (3.4) into Eqn. (3.2), the equation becomes,

$$
2\left(\frac{2 \pi}{\lambda} n_{\text {core }}^{\text {eff }}\right)=\frac{2 \pi}{\Lambda}
$$

which yields the well-known Bragg equation or phase-matching equation for fiber Bragg gratings,

$$
\lambda_{\text {res }}=2 n_{\text {core }}^{\text {eff }} \Lambda
$$


where $\lambda_{\text {res }}$ and $\Lambda$ are the reflected Bragg wavelength and grating period respectively. Eqn. (3.6) shows that the Bragg wavelength is directly proportional to the effective index of the core as well as the grating period. Hence any changes in these two terms will result in a change in the reflected Bragg wavelength.

The initial use of FBG is in the area of optical communications as an optical filter in Dense Wavelength Division Multiplexing (DWDM) network, fiber amplifier, dispersion compensation, tunable filter and external cavity laser diodes [75]. Ref. [75] gives an excellent overview of the applications of FBG in optical communications as well as sensing

Beside its application in optical communications, the FBG has also been used widely for sensing applications. Being sensitive to external perturbations, such as temperature and strain, the Bragg wavelength of the FBG fluctuates with these perturbations. Thus, this poses a problem in optical communications and therefore compensation has to be incorporated in order to correct for the errors due to these perturbations. These disadvantages however make FBG an excellent optical fiber sensor device, suitable for measuring static and dynamic parameters, such as temperature, strain and pressure [76]. Since the FBG sensor's response to perturbations is wavelength encoded, these perturbations are determined by measuring the amount of shift in the reflected Bragg wavelength with respect to the original value. Although measurements of FBG sensor's response include measuring the transmitted or reflected FBG spectrum, in most cases, measuring the reflected Bragg wavelength is preferred as this allows the configuration of single-ended probe sensors, making it convenient for use in harsh environment. The basic FBG sensor setup is illustrated in Fig. 3.3. 


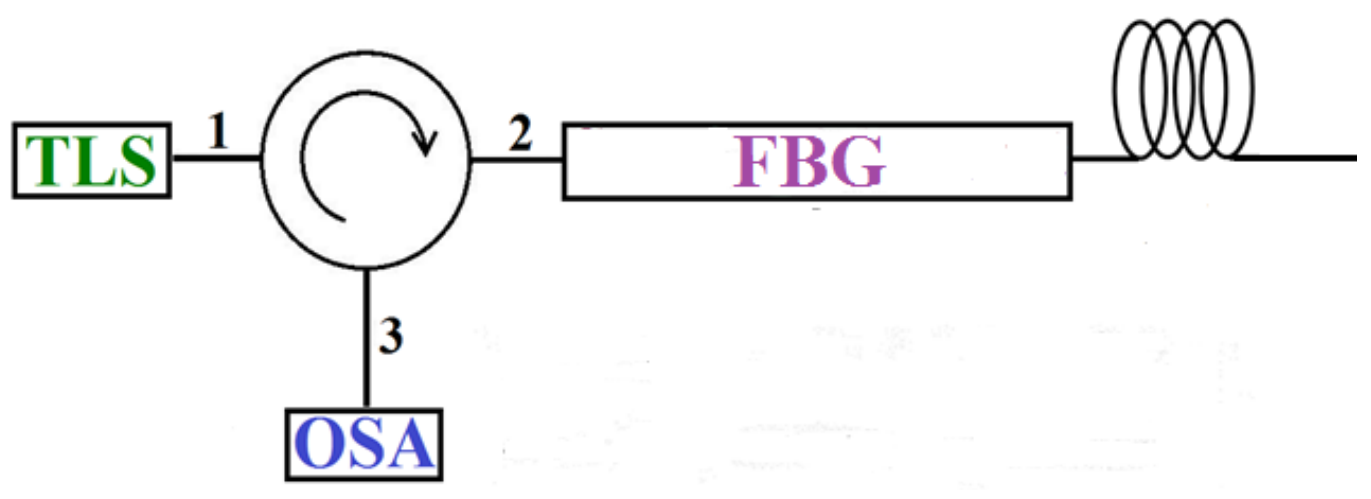

Figure 3.3 Schematic diagram of a typical FBG setup

An optical circulator, in Fig. 3.3, is an optical device that allows light to be transmitted from port 1 to port 2 but redirects any reflected light from port 2 to port 3 . The Bragg wavelength's response to external perturbation from the FBG sensor, Fig. 3.3, is monitored through an optical spectrum analyzer via port 3 of the optical circulator.

From Eqn. (3.6), the Bragg wavelength is dependent on the effective refractive index of the core and the periodicity of the grating. The effective refractive index, as well as the periodic spacing between the gratings, is affected by changes in strain, temperature and external refractive index in certain configuration. Differentiating Eqn. (3.6) with respect to external perturbation $P$ yields,

$$
\Delta \lambda=2\left(\Lambda \frac{\partial n_{e f f}}{\partial P}+n_{e f f} \frac{\partial \Lambda}{\partial P}\right) \Delta P
$$

Eqn. (3.7) corresponds to a change in the grating spacing and induced change in the refractive index due to either strain or temperature, where $P=\varepsilon$ for strain and $P=T$ for temperature. In the case of strain sensing, Eqn. (3.7) can be expressed as [76] 


$$
\Delta \lambda=\lambda\left(1-\rho_{e}\right) \varepsilon_{z}
$$

where $\rho_{e}$ is an effective strain-optic constant defined as,

$$
\rho_{e}=\frac{n_{e f f}^{2}}{2}\left[\rho_{12}-v\left(\rho_{11}+\rho_{12}\right)\right]
$$

where $\rho_{11}$ and $\rho_{12}$ are components of the strain-optic tensor and $v$ is the Poisson's ratio. For typical germanosilicate optical fiber, $\rho_{11}=0.113, \rho_{12}=0.252, \nu=0.16$ and $n_{e f f}=1.482$ [76] . Hence using these parameters and Eqs. (3.8) and (3.9), the estimated strain sensitivity at $\sim 1550 \mathrm{~nm}$ is a $1.2 \mathrm{pm}$ change resulting from applying $1 \mu \varepsilon$ to the FBG.

For a temperature induced Bragg wavelength change, Eqn. (3.7) would be written as [76]

$$
\Delta \lambda=\lambda_{\text {res }}\left(\alpha_{A}+\alpha_{n}\right) \Delta T
$$

where $\alpha_{A}=(1 / \Lambda)(\partial \Lambda / \partial T)$ is the thermal expansion coefficient for the fiber (approximately $0.55 \times 10^{-6}$ for silica). The $\alpha_{n}=\left(1 / n_{e f f}\right)\left(\partial n_{e f f} / \partial T\right)$ represents the thermo-optic coefficient, which is approximately $8.6 \times 10^{-6}$ for the germanium-doped silica core fiber.

Although the FBG sensor is able to detect numerous perturbations, there are cases where detection is possible only through modifying the physical structure of the optical fiber. For example, when using FBG sensor for refractive index sensing, the fiber cladding has to be etched to a distance close to the fiber core in order to gain access to the evanescent field propagating at the core-cladding interface [77, 78]. Recall Eqn. (3.6), the effective refractive 
index of the core is affected by the external refractive index only when the fiber cladding is etched or polished so that evanescent wave can propagate out of the fiber. Once $n_{\text {eff }}$ is changed resulting from a change of external RI, the Bragg wavelength will also change accordingly. The disadvantage of etching or polishing is that the fiber's mechanical strength is reduced. An alternative to such technique is to inscribe gratings in a microfiber.

In this work, a double-cladded photosensitive single-mode fiber as well as multimode fiber with gradient-index profile were studied for the microfiber Bragg gratings.

\subsubsection{Microfiber Bragg gratings}

i. Single-mode fiber as the preform of the microfiber

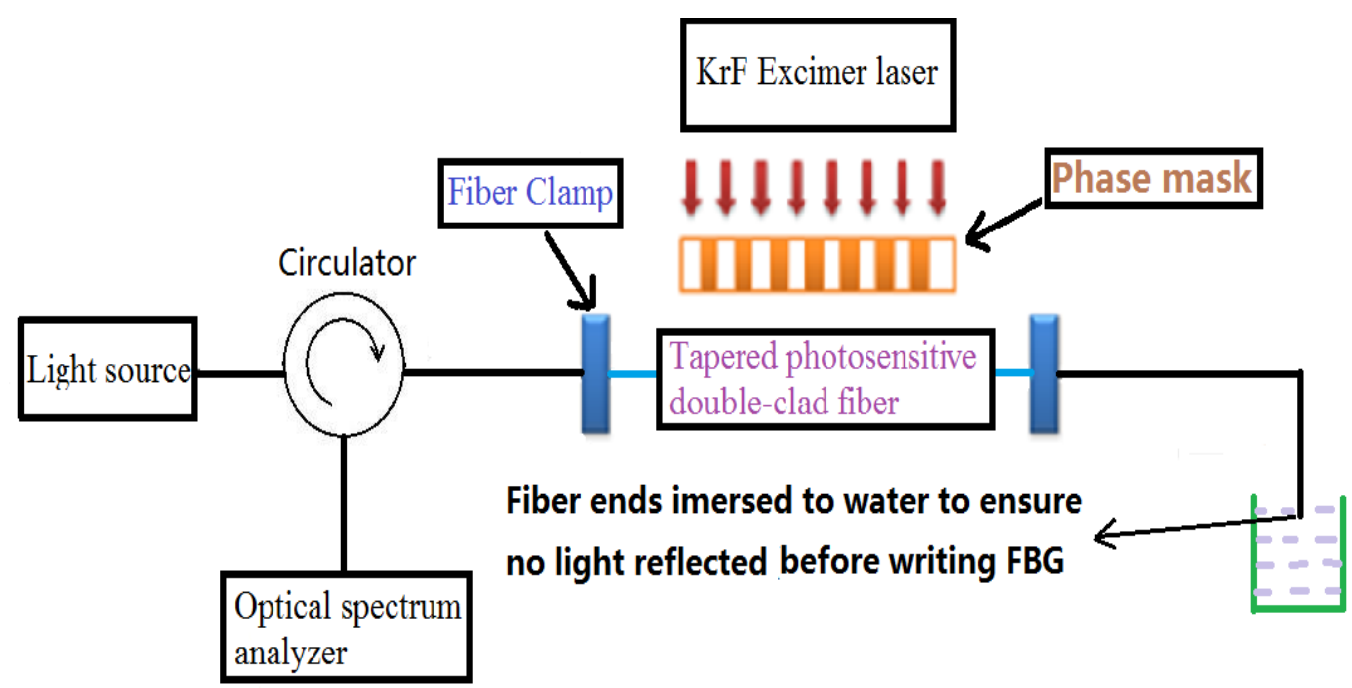

Figure 3.4 Experimental setup of fabrication of microfiber Bragg gratings 
The preform for the microfiber is an SMF of 3.6- $\mu \mathrm{m}$-diameter highly Ge-doped core, 25 $\mu \mathrm{m}$-diameter $\mathrm{Ge} / \mathrm{B}$ co-doped inner cladding, and 125 - $\mu \mathrm{m}$-diameter undoped outer cladding. The microfiber was tapered from such an SMF preform using the fiber tapering machine. For the fabrication of the MFBG, we used a $248 \mathrm{~nm} \mathrm{KrF}$ excimer laser with $25 \mathrm{~ns}$ pulses having energy of $200 \mathrm{~mJ} /$ pulse at a pulse repetition rate of $10 \mathrm{~Hz}$, through a $1 \mathrm{~cm}$ long uniform phase mask with a pitch of $1082.3 \mathrm{~nm}$. The experimental set-up is shown in Fig. 3.4 .

Fig. 3.5 shows the schematic diagram of a microfiber with a diameter of $7.6 \mu \mathrm{m}$ and the MFBG written in the central region of the microfiber. The scanning electron microscope (SEM) image of the microfiber inscribed with a grating is shown in Fig. 3.6.

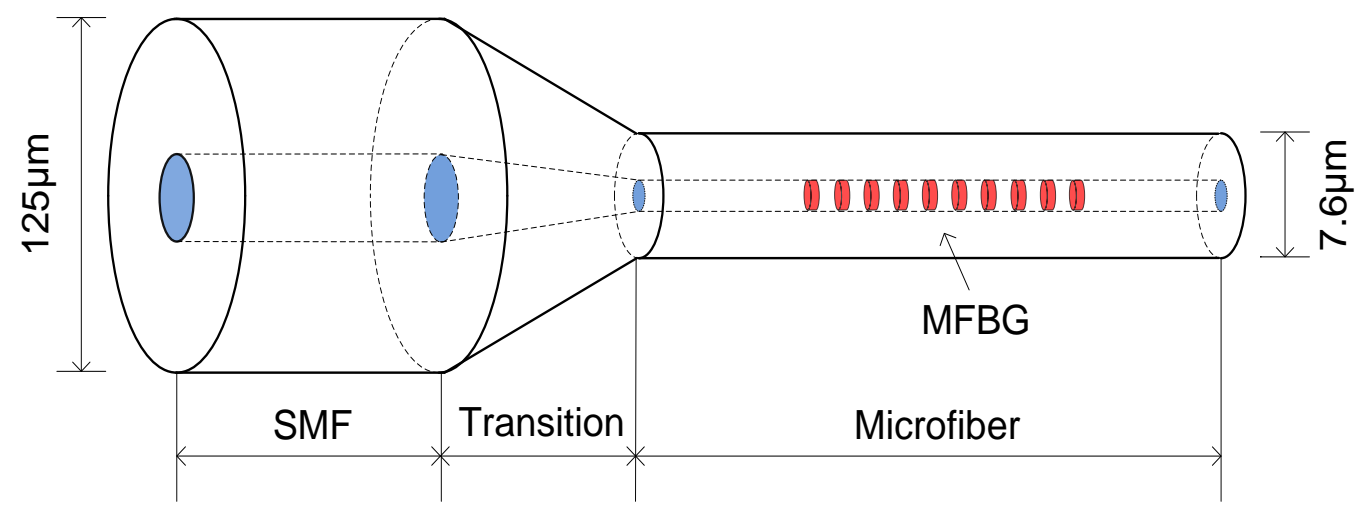

Figure 3.5 Schematic diagram of microfiber Bragg gratings 


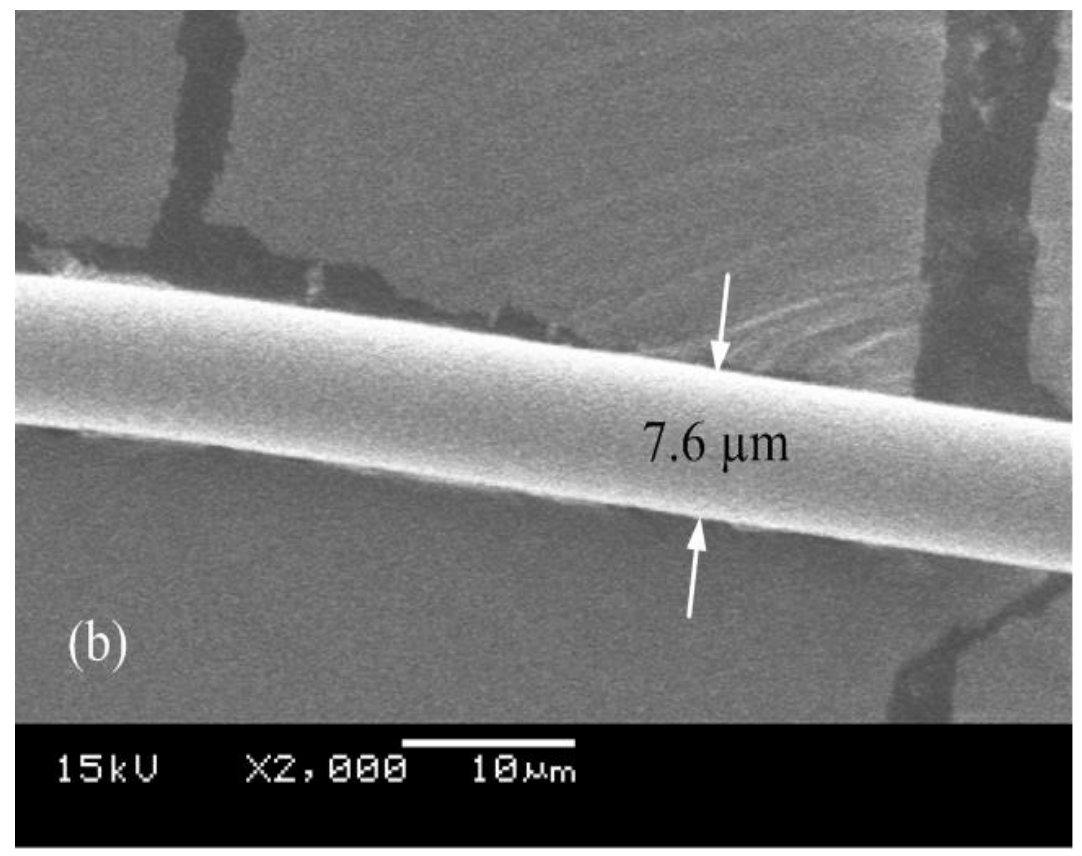

Figure 3.6 SEM image of the microfiber.

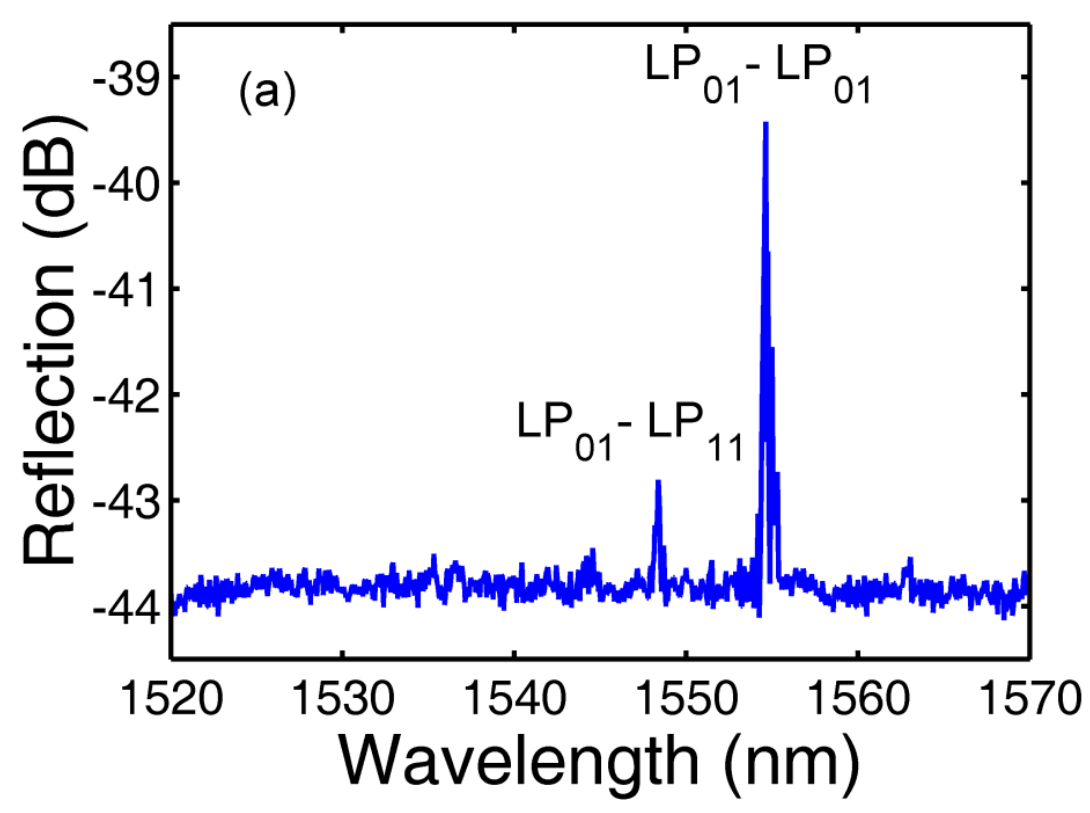

Figure 3.7 Reflection spectrum of an MFBG with a diameter of $7.6 \mu \mathrm{m}$ using single-mode fiber. 
An Ando 4321D tunable laser source (TLS) and an Ando AQ 6317B optical spectrum analyzer (OSA) were used to monitor the reflection spectrum of the MFBG. During the grating fabrication process, we found that there was obvious monotonic increase in the reflectivity of the reflected spectrum, proving that the MFBG is a type I grating. Two reflected peaks were observed in Fig. 3.7. The peak at $1554.64 \mathrm{~nm}$ is due to mode coupling of the counter propagating fundamental $\mathrm{LP}_{01}$ modes, while the other peak at $1548.29 \mathrm{~nm}$ is a result of the coupling of the counter propagating fundamental $\mathrm{LP}_{01}$ and the higher order $\mathrm{LP}_{02} / \mathrm{LP}_{11}$ modes [79]. The reason for generation of such higher order mode attributes to the dispersion of the germanium doping in the fiber core into the cladding during the tapering process. In this case, the Bragg gratings would be also formed in certain area of the cladding.

For RI sensing application, a commercial refractometer (KEM Ra-130) was used as a reference to determine the ambient RI of the fluid in which the MFBG is immersed in. To obtain a range of RI units, we dissolved different measured quantities of sugar in distill water to achieve RI values ranging from 1.3332 to 1.4481 . Fig. 3.8 shows the peak wavelength shift as a function of the ambient RI. It can be seen that, the reflection spectrum red shifts as the ambient RI increases for both of the two reflection peaks. Also, a relatively high RI sensitivity is achieved when the ambient RI approaches the RI value of the fiber cladding. This is reasonable since the mode field propagated within the fiber is less confined in the microfiber and hence more easily affected by the RI of the surrounding medium. The RI sensitivity is determined from the gradient of the exponentially fitted curve shown in Fig. 3.8. The fundamental mode resonance wavelength is determined to have a maximum sensitivity of about $79.9 \mathrm{~nm} / \mathrm{RIU}$ (refractive index unit) at $\mathrm{RI}=1.4481$. 


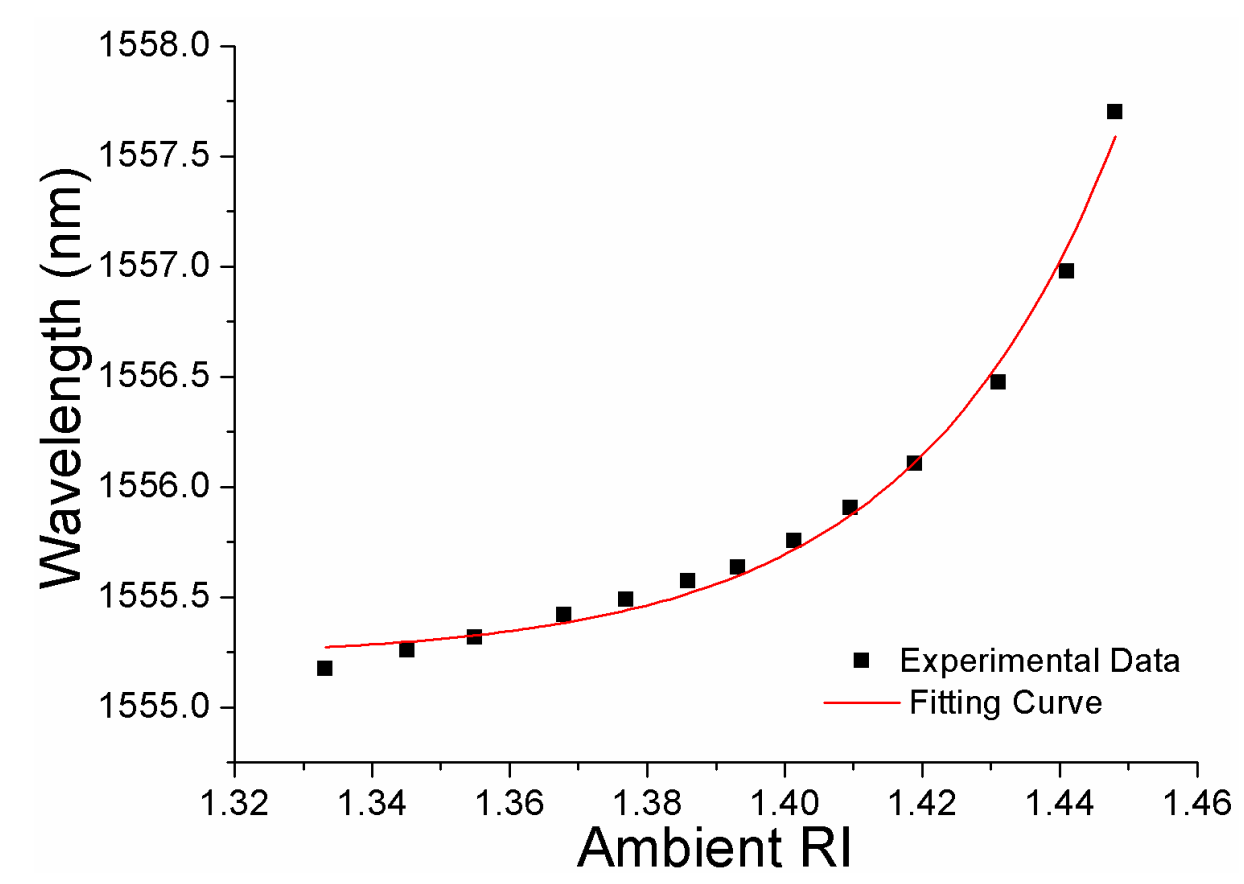

Figure 3.8 Wavelength versus the RI of the surrounding medium.

\section{ii. Multi-mode fiber as the preform of the microfiber}

We also investigated MFBG using multi-mode fiber. Since the core diameter of multi-mode fiber is normally very large, it is also suitable for tapered fiber applications. The fiber used in this section is a graded-index fiber, with core diameter of $50 \mu \mathrm{m}$. The core has germanium doping and numerical aperture is 0.22. Fig. 3.9 shows the reflection spectrum of an MFBG with a diameter of $7.5 \mu \mathrm{m}$. Only one reflection peak was observed, which is due to mode coupling of the counter propagating fundamental $\mathrm{HE}_{11}$ modes. Multiple sidelobes are generated due to the graded-index profile of such multi-mode fiber. Fig. 3.10 shows the reflection spectrum of an MFBG with a diameter of $6.4 \mu \mathrm{m}$. It was observed that the left peak has larger shift than the right peak when the external refractive index increases. This is because the left peak is the resonant wavelength of higher-order mode which will have lower 
effective refractive index. In addition, the variation of external refractive index will have a larger impact on the higher-order mode's effective index.

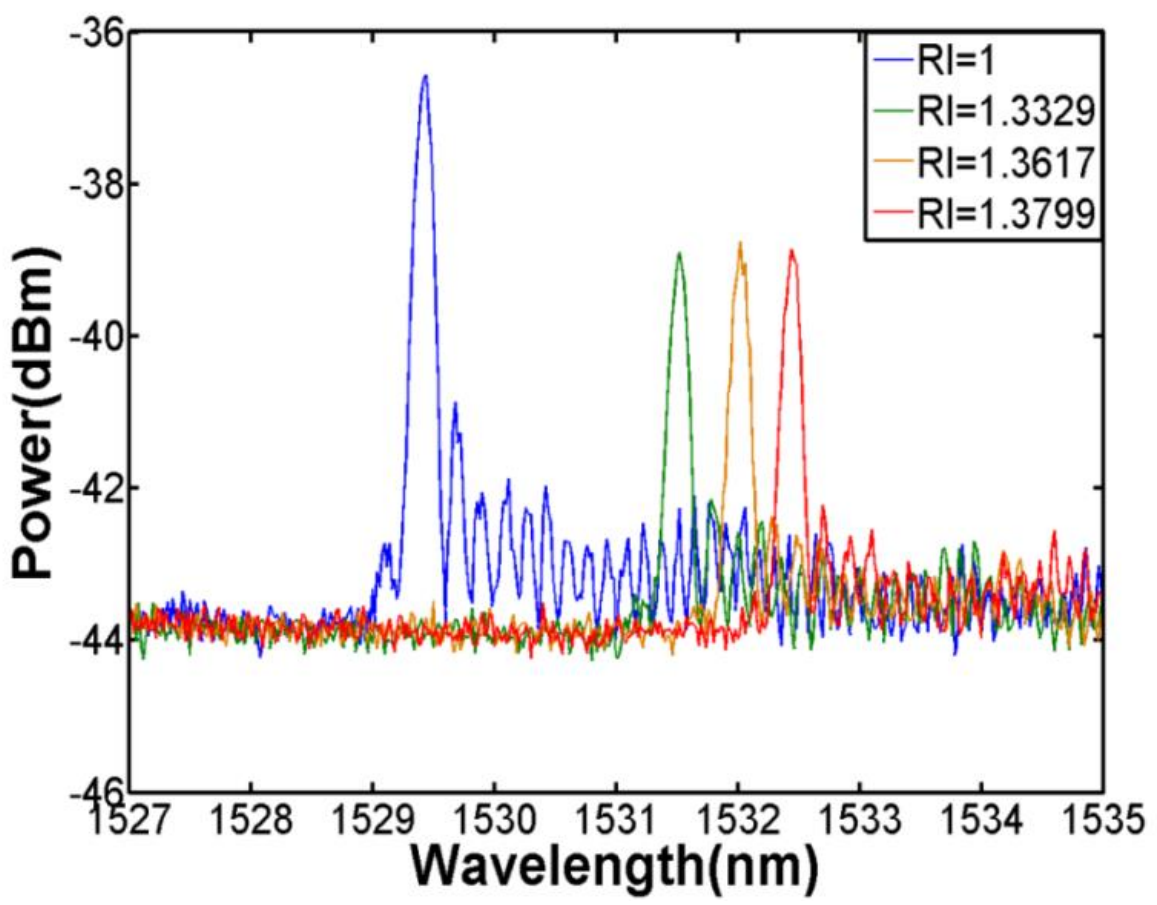

Figure 3.9 Reflection spectrum for different RI for MFBG with diameter of $7.5 \mu \mathrm{m}$ 


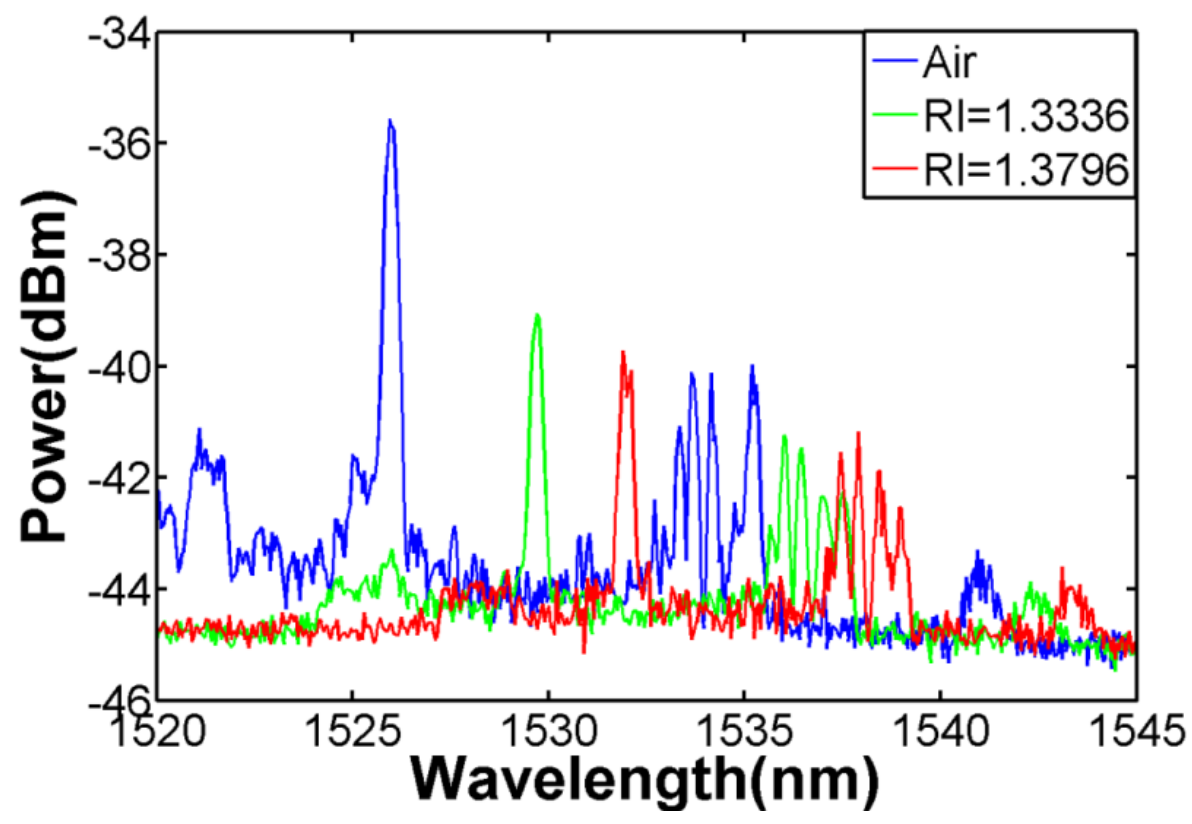

Figure 3.10 Reflection spectrum for different RI for MFBG with diameter of $6.4 \mu \mathrm{m}$

\subsection{Microfiber long period gratings}

\subsubsection{Introduction of long period gratings}

Long period gratings transfer power between the fundamental core mode and cladding modes under phase-matching condition is depicted in Fig. 3.11. 


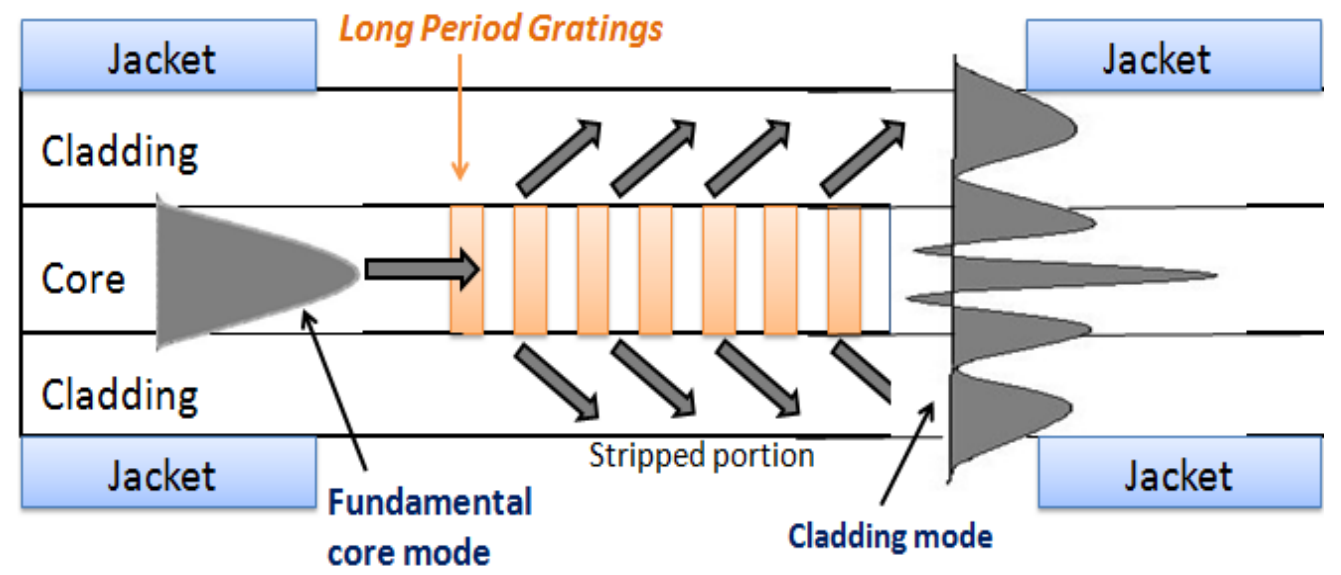

Figure 3.11 Schematic diagram of mode evolving in LPG

For normal LPGs, the phase matching condition of coupling power between higher order mode and fundamental mode is shown to be [80]

$$
\left|\beta_{1}-\beta_{2}\right|=\frac{2 \pi}{\Lambda}
$$

where $\beta_{1}$ and $\beta_{2}$ are the propagation constants of the fundamental mode and higher order mode, $\Lambda$ is the period of the gratings. With $\beta_{1}=n_{\mathrm{co}}^{e f f} k$ and $\beta_{2}=n_{\text {clad }, m}^{e f f} k$, it can be easily derived from Eqn. (3.11) that the resonant wavelength under this phase matching condition takes the form

$$
\lambda_{\text {res }}=\left(n_{\mathrm{co}}^{\text {eff }}-n_{\mathrm{clad}, m}^{\text {eff }}\right) \Lambda
$$

where $\lambda_{\text {res }}$ is the resonance wavelength, $n_{\mathrm{co}}^{\text {eff }}$ is the effective refractive index of the core mode and $n_{\text {clad, } m}^{e f f}$ is the effective refractive index of the $m$ th cladding mode. The wavelength dependence of the effective refractive indices is due to material and 
waveguide dispersion. Guide light in the fundamental $\mathrm{LP}_{01}$ core mode is perturbed by the presence of the gratings in the fiber core. The difference between the propagation constant of the guided core mode and the phase-matching vector of the grating equals the propagation constant of one or more cladding modes at the appropriate wavelengths. Cladding modes are then guided in the cladding region along the cladding-air interface and the modes attenuate rapidly due to bends in the optical fiber and absorption by the cladding material. Therefore, energy of the cladding mode at phase-matched wavelength is lost from the fiber. The result is series of resonance dips or bands in the LPG transmission spectrum. Differentiating Eqn. (3.12) that we get

$$
\frac{d \lambda_{r e s}}{d \Lambda}=\frac{n_{c o}^{e f f}-n_{c l a d, m}^{e f f}}{1-\Lambda \frac{d\left(n_{c o}^{e f f}-n_{c l a d, m}^{e f f}\right)}{d \lambda_{r e s}}}
$$

From Eqn. (3.13), it can be clearly seen that the resonant wavelength of a cladding mode, as a result of the coupling from the core mode, can be predicted by a particular period. The first step of prediction involves the calculation of the effective refractive indices of the fundamental core mode and the various resonant cladding modes of the fiber for a specific wavelength. 


\subsubsection{Experimental results}

In this work, the fibers, which were tapered to $60 \mu \mathrm{m}$ and $55 \mu \mathrm{m}$, were both adiabatically stretched in order to obtain a flat transmission spectrum. It is crucial to ensure that the fiber is tapered gradual enough, otherwise interference will occur [39]. The taper transition lengths are around $10 \mathrm{~mm}$ each and the taper waist length is around $20 \mathrm{~mm}$.

After tapering, the fiber was exposed to a $248 \mathrm{~nm} \mathrm{KrF}$ excimer laser with a pulse frequency of $10 \mathrm{~Hz}$ and fluence of $200 \mathrm{~mJ} / \mathrm{cm}^{2}$ to fabricate the LPG. The periods of the amplitude mask for diameters of $60 \mu \mathrm{m}$ and $55 \mu \mathrm{m}$ are $320 \mu \mathrm{m}$ and $450 \mu \mathrm{m}$, respectively. Fig. 3.12 shows a schematic diagram of the LPG fabrication setup. The single-mode fiber used in the experiment is the photosensitive double-clad fiber with High Ge-doped core $(22 \mathrm{~mol} \%)$ whose diameter is around $3.6 \mu \mathrm{m}$. The inner cladding has a diameter of $25 \mu \mathrm{m}$ and is lightly doped with Ge and Boron (B) (1 mol\%). The co-doping of Boron in germanosilicate fiber enhances UV photosensitivity of the fiber $[81,82]$. The remaining area is the outer cladding with diameter of $125 \mu \mathrm{m}$. The refractive index distribution profile is $\mathrm{W}$-shaped as shown in Fig. 3.13. The core has the highest RI while the inner cladding has the lowest. In the tapered fiber with waist diameter of $60 \mu \mathrm{m}$, the core diameter is reduced to 1.73 $\mu \mathrm{m}$ while the inner cladding diameter becomes $12 \mu \mathrm{m}$. To further enhance the

photosensitivity, the tapered fibers were loaded with hydrogen at a temperature of $70^{\circ} \mathrm{C}$ and a pressure of 2000 psi for 192 hours. The resonant dips of the two LPGs were observed at around 2000 pulses of irradiation. After the gratings were written, 
the fibers were annealed at $120^{\circ} \mathrm{C}$ for 20 hours to dispel the hydrogen that may still be present in the fiber. The reason for choosing such a double-cladded fiber is that the photosensitive inner cladding could play the role of new "core" after tapering. This will ensure that the gratings could be effectively inscribed into the tapered microfiber as the original core diameter almost vanishes.

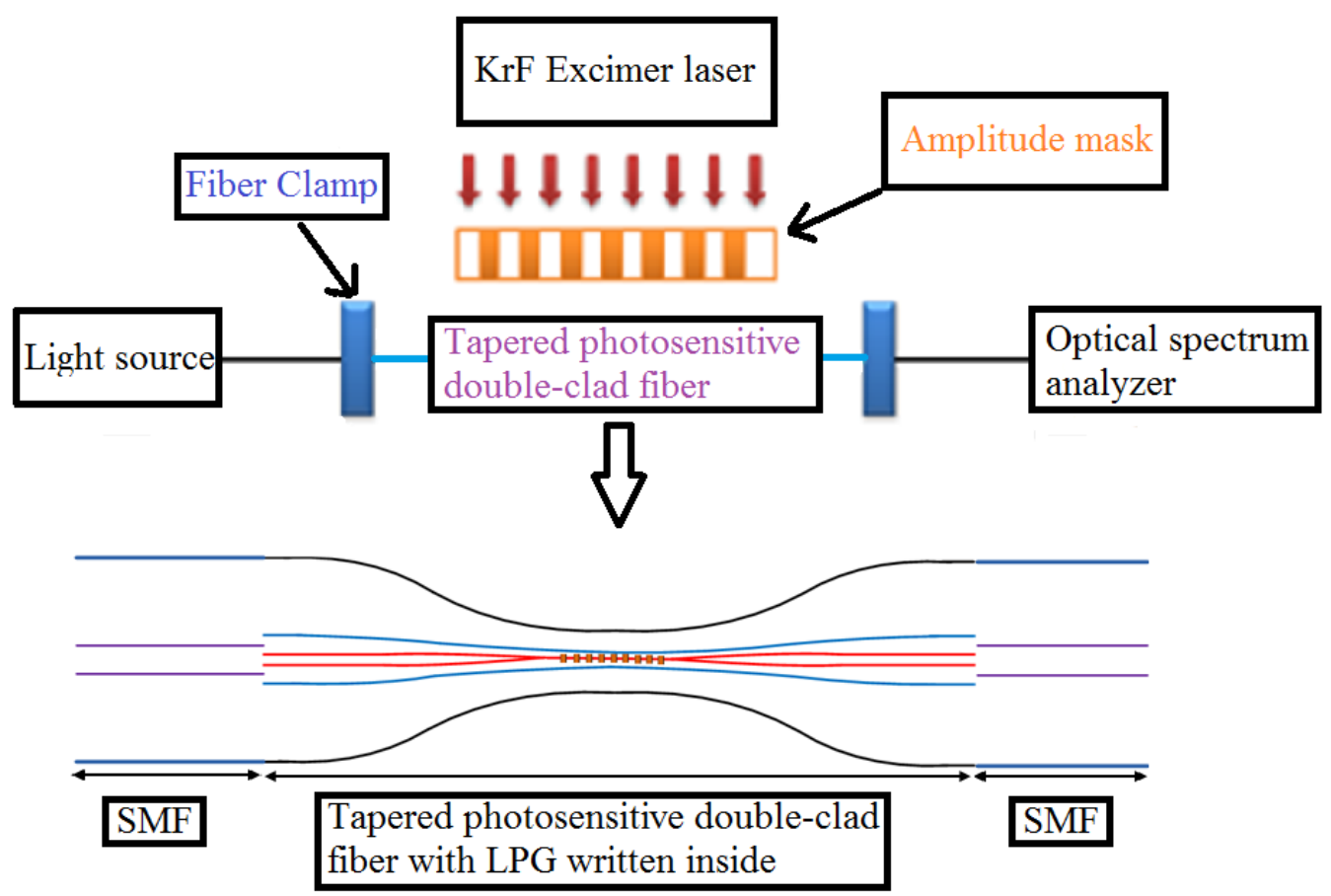

Figure 3.12 Schematic diagram of the experimental set-up 


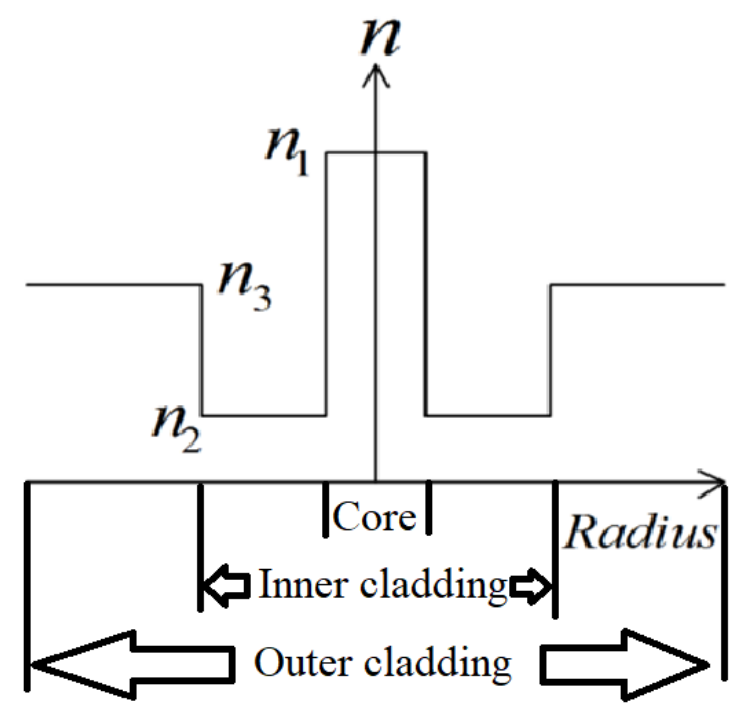

Figure 3.13 Refractive index profile of the W type double-clad fiber

\section{i. $\quad$ Refractive index sensing}

To characterize the refractive index sensitivity of the sensor, a few drops of salt solution were dripped onto the sensing region, which is the taper waist. The salt solutions with different refractive indices were prepared by diluting the saturated $\mathrm{NaCl}$ solution using distilled water. The refractive index of the solution was determined using a refractometer (KEM RA-130) with a resolution of 0.0001 . The resulting wavelength responses are depicted in Fig. 3.14. In contrast to the expected behavior of the transmission spectrum for conventional LPGs, we noted that the resonant dip of the fabricated LPG shifts towards the longer wavelength when the external refractive index increases. The main reason for such phenomenon is due to the $\mathrm{W}$-shaped refractive index profile of the fiber as discussed in the aforementioned section. After tapering, the original core diameter of $3.6 \mu \mathrm{m}$ would have become smaller and does not contribute significantly to $n_{\mathrm{eff}}$ of the core mode. In addition, it 
is believed that bulk of the Ge in the fiber core have dispersed into the inner cladding during the tapering process but hardly into the outer cladding [79]. In other words, for the tapered fiber, the effective refractive index of the core mode after tapering is lower than the refractive index of the outer cladding. The phase matching condition for coupling between the higher-order modes to fundamental mode can still be derived from Eqn. (3.11), but the resonance wavelength is now given by

$$
\lambda_{\text {res }}=\left(n_{\text {clad }, m}^{e f f}-n_{c o}^{e f f}\right) \Lambda
$$

Based on the data provided by the manufacturer for the W-shaped specialty fiber, the refractive index of the core is 1.4650 , the inner cladding is 1.4403 , and the outer cladding is 1.4476 . The guiding mechanism after tapering was also simulated. It was found that such tapered specialty fiber still supports single mode only. The mode field diameter of the new core mode is calculated to be $4.43 \mu \mathrm{m}$ which is much larger than the new core diameter of $1.73 \mu \mathrm{m}$, but much smaller than the new inner cladding diameter of $12 \mu \mathrm{m}$. Therefore, although the refractive index of the core is still much higher than the inner cladding and outer cladding, the effective index of the core mode will be greatly influenced by the inner cladding. As expected, the effective refractive index of the core mode was calculated to be 1.4443 which is smaller than refractive index of the cladding. This can justify the validity of Eq. (3.14). For this simulation, it is not possible to estimate the percentage of Ge, originally in the core, that has diffused to the inner cladding. Therefore, the value of the effective index 1.4443 may not be accurate enough. However, since after tapering, the inner cladding size is much larger than the core size, the increase of the refractive index of the inner 
cladding caused by diffusion is nearly negligible. Now it can be seen from Eqn. (3.14) that the resonant wavelength will shift to a longer wavelength due to increase of external refractive index.

A wider spectral plots are shown in Figure 3.15 from which one can see that the couplings between the fundamental mode and the higher-order modes that have closer propagation constants with fundamental mode are very weak. This is because the power of the modes in the inner cladding is weak due to the higher refractive index of the outer cladding. The transmission loss becomes smaller as the liquid RI increases. This phenomenon is not related to adiabaticity of the taper. For LPG, the transmission loss for the resonant wavelength will disappear when $n_{\text {ext }}=n_{c l}$ since the core will see an infinitely thick cladding region. In such case, there is no cladding-surrounding interface and thus the cladding modes do not exist [83-85]. Therefore, when the external refractive index gets closer to that of cladding, less power of cladding modes will be converted to power of radiation modes, resulting in a diminutive loss in the transmission spectrum.

When the external refractive index increases, the effective index of the higherorder mode will increase more than the fundamental mode. Now it can be seen from Eqn. (3.14) that the resonant wavelength will have a red shift due to the increase of the external refractive index. The maximum RI sensitivity achieved is around $600 \mathrm{~nm} / \mathrm{RIU}$. With our OSA's resolution of $10 \mathrm{pm}$, the detection limit for refractive index is $1.67 \times 10^{-5}$. Fig. 3.16 illustrates the sensitivity characterization of two sensors with different tapered diameters. The slope of the fiber with smaller outer diameter 
$(55 \mu \mathrm{m})$, plotted using squares, and linearly fitted by purple line is slightly larger than the slope of the fiber with larger outer diameter, plotted using circles. It was unfortunate that the supply of the double-cladd W-shaped photosensitive specialty fiber was no longer available in the market. The author could not continue a more thorough work on investigating the sensitivity for MLPG with thinner diameters.

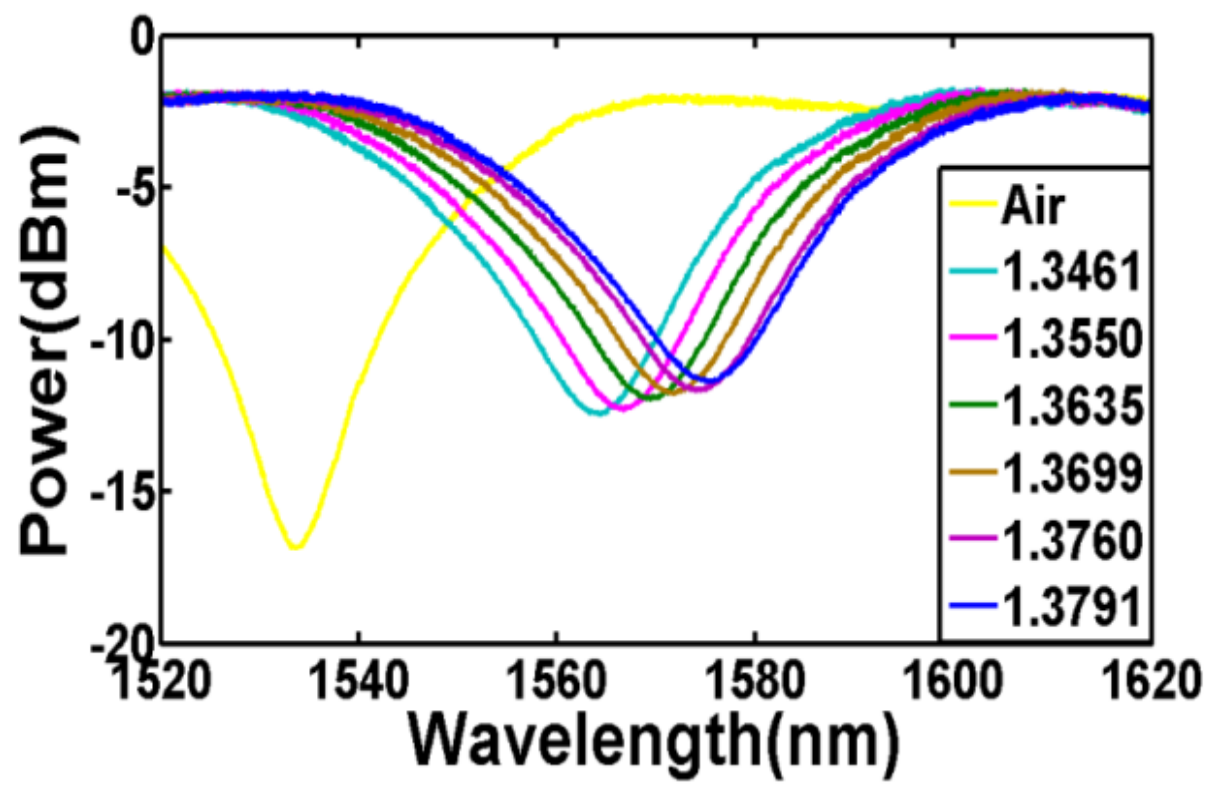

Figure 3.14 Wavelength response for different external refractive index $(D=60 \mu \mathrm{m})$ 


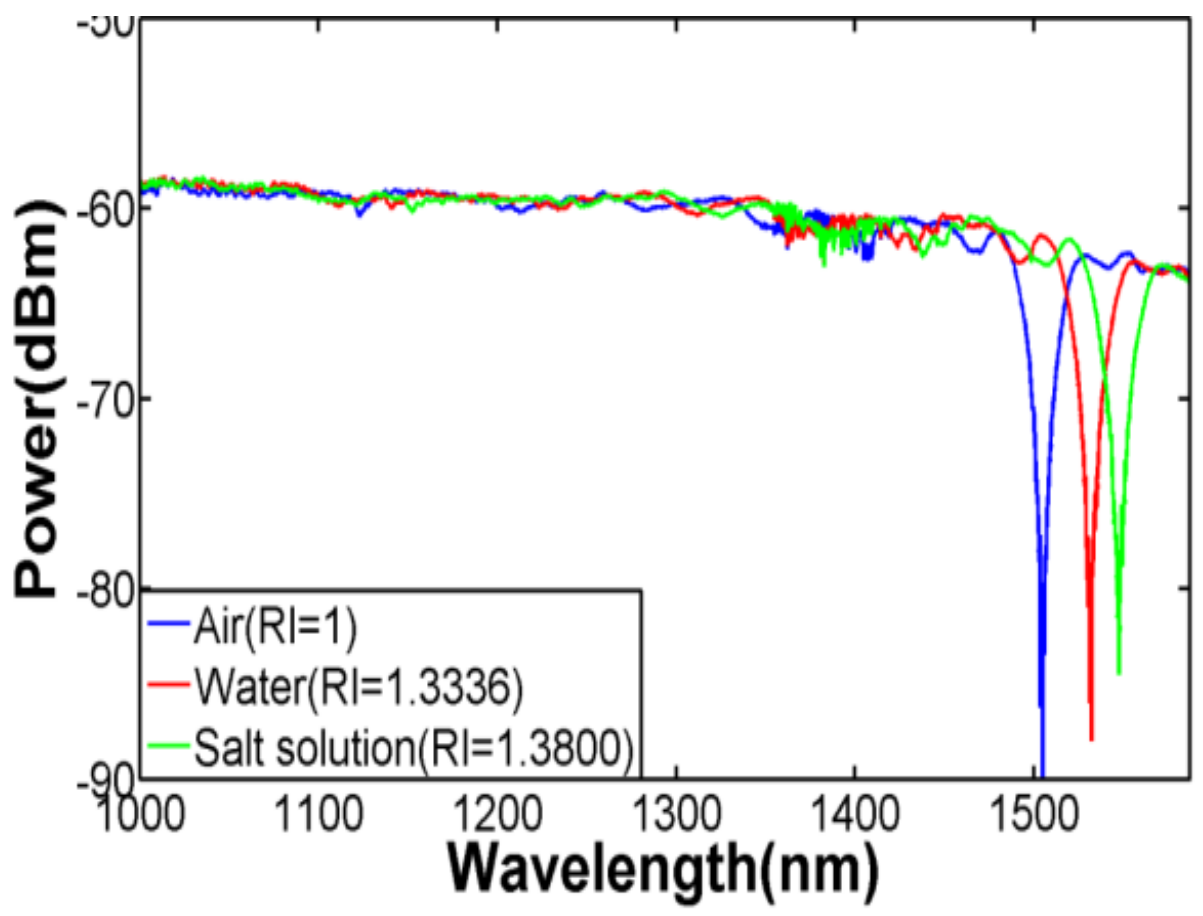

Figure 3.15 Wavelength response for different external refractive index $(D=55 \mu \mathrm{m})$

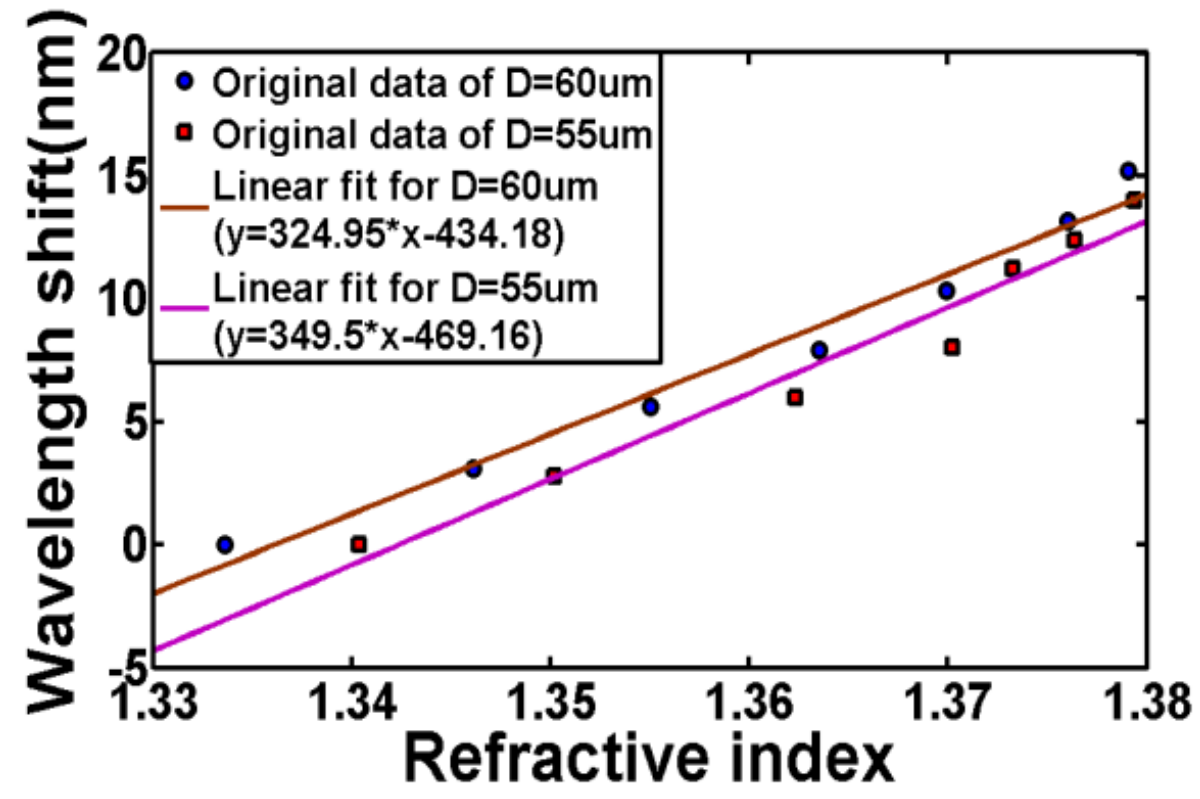

Figure 3.16 RI sensitivity characterization for microfibers with diameters of $60 \mu \mathrm{m}$ and $55 \mu \mathrm{m}$ 


\section{ii. $\quad$ Temperature sensing}

For temperature sensing, the sensitivity of an LPG sensor depends not only on the orders of the cladding mode but also the dopant used for optical fiber. It has been shown that the B/Ge co-doped fiber has much higher temperature sensitivity than the normal single-mode fiber SMF28 $[80,86]$. This can be illustrated by using chain rule of derivatives, the sensitivity to a temperature $T$ for a grating of length $L$ can be obtained from Eqn. (3.14) [87]

$$
\frac{d \lambda_{\text {res }}}{d T}=\frac{\left(d n_{\mathrm{cl}, m}^{e f f}-d n_{\mathrm{core}}^{e f f}\right)}{d T} \Lambda+\left(n_{\mathrm{cl}, m}^{e f f}-n_{\mathrm{core}}^{e f f}\right) \frac{d \Lambda}{d T}
$$

Eqn. (3.15) shows that there are two factors affecting the temperature sensitivity of LPGs. One is the material contribution due to the change in the differential refractive index of the core and cladding modes arising from the thermo-optic effect. The other is the waveguide contribution which is the change in the grating periodicity due to thermal expansion of the fiber. For grating period of hundreds of micrometers, the material contribution will dominate the waveguide contribution [88] which applies in this work since the grating period adopted is greater than $400 \mu \mathrm{m}$. The presence of boron alters the temperature dependence of the refractive index. Compared with germanosilicate fiber, the difference in thermo-optic coefficients for the $\mathrm{B} / \mathrm{Ge}$ fiber is higher so that the sensitivity is also higher. Researchers have achieved high temperature sensitivity of $2.75 \mathrm{~nm} /{ }^{\circ} \mathrm{C}$ using such fiber [89]. Fig. 3.17 shows the wavelength response for different temperatures. It can be seen from Fig. 3.18 that the wavelength shift is around $4 \mathrm{~nm}$ when the temperature varies from 20 
${ }^{\circ} \mathrm{C}$ to $90{ }^{\circ} \mathrm{C}$. The sensitivity is not as high as many $\mathrm{B} / \mathrm{Ge}$ fibers, and this may be due to the fact that the doping concentration of Boron in our fiber is low (only $1 \mathrm{~mol} \%$ ). However, it is still comparable with LPGs sensors written in germanosilicate fiber.

Another interesting point to note is that the resonant wavelength also shifts to the right (red shift) when the temperature increases, which is similar to the response to increasing ambient refractive index described in the previous section. In the case of conventional B/Ge fiber, the wavelength will shift towards the left (blue shift) when the grating period is large enough which indicates that the material contribution dominates. This can be explained if we recall Eqn. (3.15). For conventional B/Ge fiber, the term $d n_{\mathrm{cl}, m}^{e f f}-d n_{\mathrm{core}}^{e f f}$ should be $d n_{\mathrm{core}}^{e f f}-d n_{\mathrm{cl}, m}^{e f f}$, and because the $d n / d T$ of the boronco-doped silica glass is less than that of the pure silica [87], $d \lambda / d T$ will thus be negative. However, in our case the two terms $n_{\mathrm{core}}^{\text {eff }}$ and $n_{\mathrm{cl}, m}^{\text {eff }}$ are swapped since the new core's effective index is smaller than the cladding. 


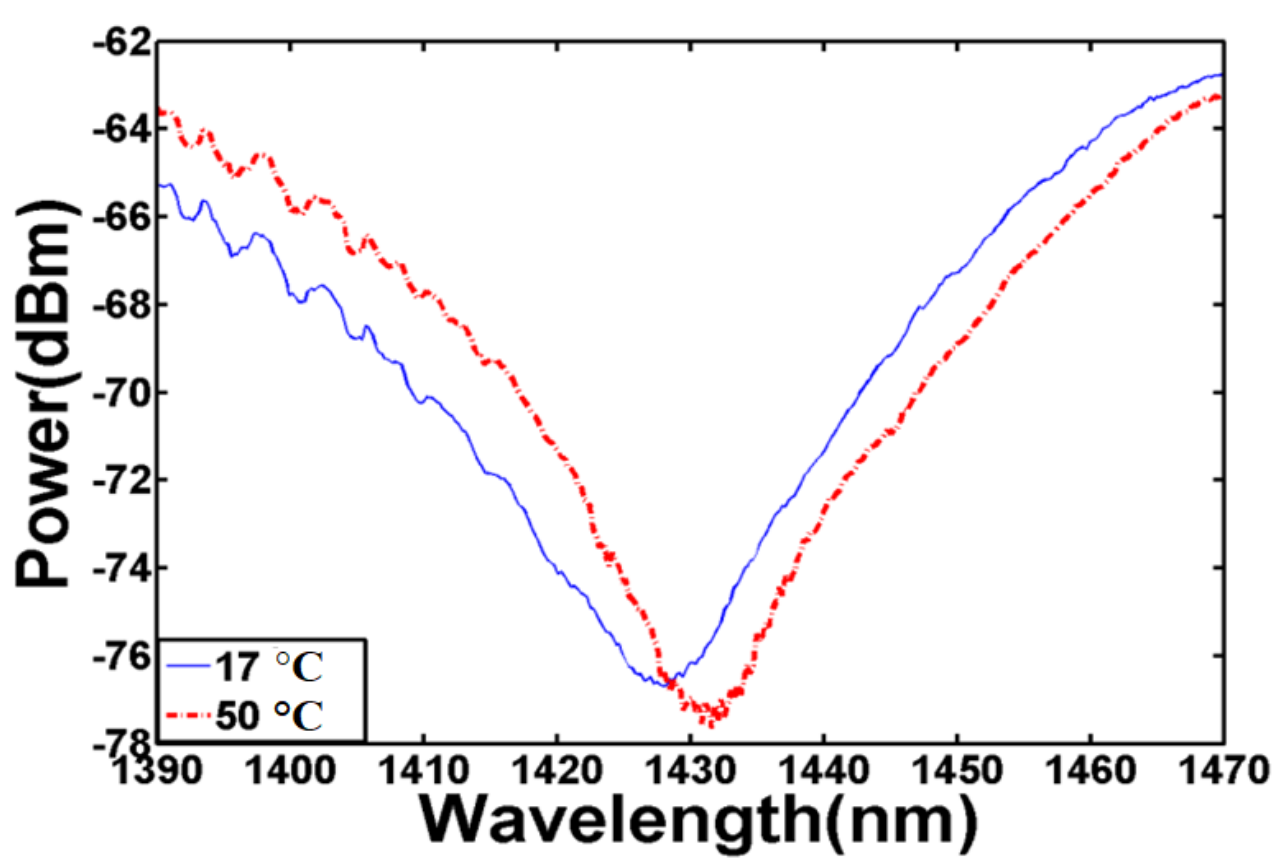

Figure 3.17 Wavelength response for different temperature $(D=55 \mu \mathrm{m})$

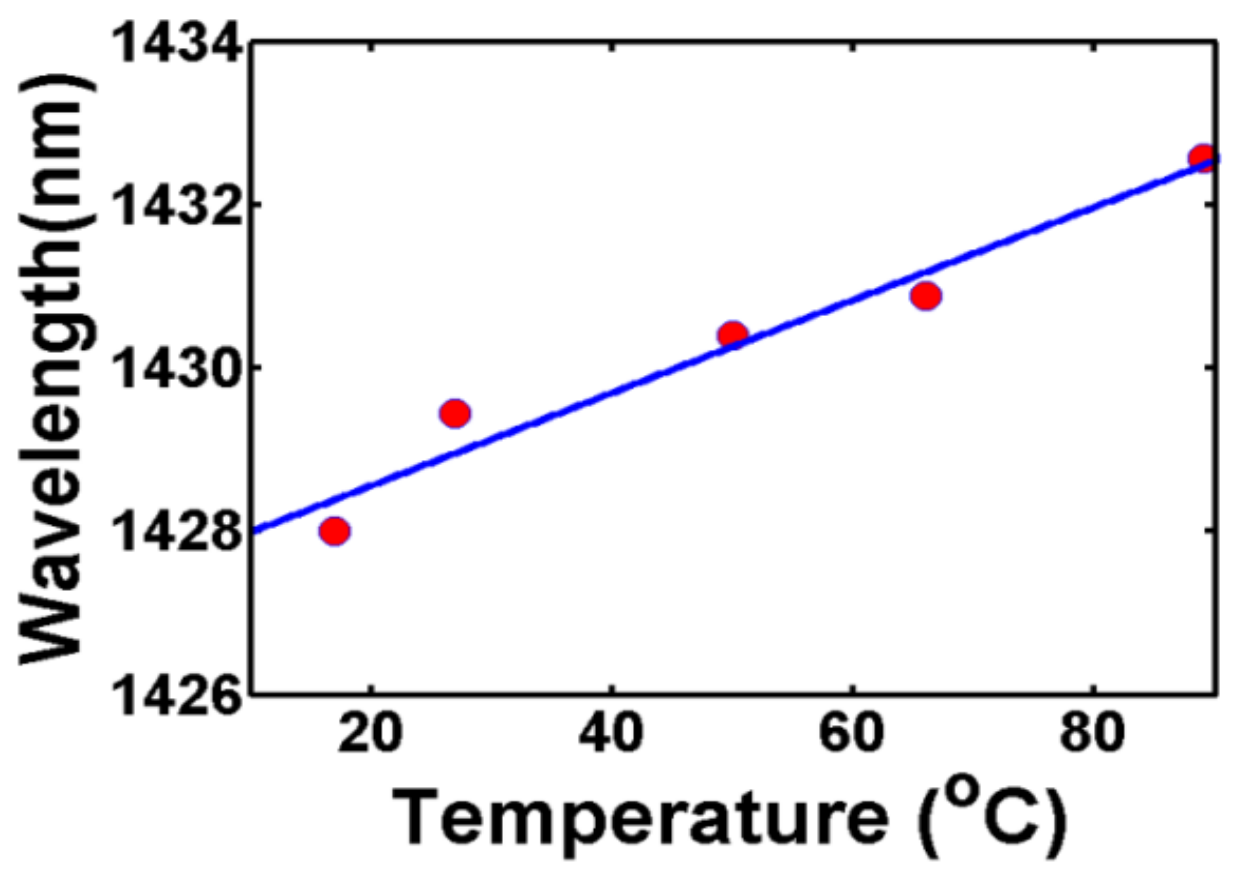

Figure 3.18 Temperature sensitivity characterization $(D=55 \mu \mathrm{m})$ 


\section{Chapter 4 : Non-adiabatically tapered microfiber sensors}

\subsection{Numerical simulation}

In this chapter, a non-adiabatic microfiber-based refractive index sensor with ultra-high sensitivity is reported using a piece of standard single-mode fiber tapered to around $4 \mu \mathrm{m}$ with large taper slope. The taper drawing machine used was the same as in previous experiments. The taper transition lengths and taper waist length were set to be less than 5 $\mathrm{mm}$ which were much shorter than the adiabatic microfibers in Chapter 3. In comparison to the microfiber mode interferometer [90-92], our sensor has a smaller diameter which makes it more sensitive to changes in the ambient refractive index. In general, large extinction ratio or peak-to-peak value leads to less reading errors and more accurate measurement. One way is to fabricate the microfiber with sharp taper slope which causes greater phase difference between fundamental mode and higher order modes. And another way is to monitor the reflection spectrum rather than the conventional transmission spectrum. This is the approach adopted for this work. In the reflection spectrum, the mode coupling in the tapered region will occur twice in the reflection based configuration so that the extinction ratio is nearly twice as compared to transmission based configuration. Therefore, by utilizing such doublepass interferometric configuration, sharper resonant peaks can be observed in the spectrum analyzer and the reading errors will be reduced. It is also worth mentioning that generally, the smaller the diameter of the microfiber, the higher the sensitivity of the sensor, as a greater portion of the core mode energy gets coupled to the evanescent field which penetrates into the surrounding medium. For adiabatic microfiber, it is difficult to reduce the diameter to the scale of a few micrometers since the taper transition needs to be sufficiently long in order 
to minimize coupling between fundamental mode and higher-order modes. On the other hand, the abruptly tapered fiber can be fabricated to a much smaller diameter and hence high sensitivity can be achieved.

For a non-adiabatic biconical fiber taper, it has been shown that most of the light energy from the fundamental mode of the untapered region will couple into the first two modes $\mathrm{HE}_{11}$ and $\mathrm{HE}_{12}$ in the tapered region [93]. As a result, the modal interference spectrum can be expressed as

$$
I=I_{1}+I_{2}+2 \sqrt{I_{1} I_{2}} \cos \varphi
$$

where $I_{m}(\mathrm{~m}=1,2)$ is the power coupling between the fundamental mode and the $\mathrm{HE}_{1 \mathrm{~m}}$ mode, and $\varphi$ is the phase difference between the two modes. For a uniform beating region, it takes the form

$$
\varphi=\Delta \beta L
$$

where $L$ is the length of the beating region, and $\Delta \beta$ is the difference between the propagation constants of the two modes, which can be theoretically represented as [94]

$$
\Delta \beta=\frac{\lambda\left(U_{2}^{\infty 2}-U_{1}^{\infty 2}\right)}{4 \pi \mathrm{n}_{\text {clad }} \rho^{2}} \exp \left(-\frac{2}{V}\right) .
$$

In Eq. (4.3), $V$ represents the normalized frequency given by 


$$
V=\frac{2 \pi \rho}{\lambda} \sqrt{\mathrm{n}_{\text {clad }}^{2}-n_{\text {ext }}^{2}}
$$

where $\mathrm{n}_{\text {clad }}$ is the refractive index of the fiber cladding, $n_{\text {ext }}$ is the refractive index of the external medium, $\lambda$ is the wavelength of light, $\rho$ is the radius of the taper waist, and $U_{1}^{\infty}$ and $U_{2}^{\infty}$ are the asymptotic values of the $U$ parameters of the two modes in which $U_{1}^{\infty}\left(\mathrm{HE}_{11}\right)=2.405$ and $U_{2}^{\infty}\left(\mathrm{HE}_{12}\right)=5.520$ in this case [95]. From the above expressions one can predict that the power spectrum is a close-form of sinusoidal function of wavelength. The free spectral range (FSR) can also be locally defined as a function of wavelength as [93]

$$
\operatorname{FSR}(\lambda)=\frac{2 \pi}{\frac{\mathrm{d} \varphi}{\mathrm{d} \lambda}}=\frac{8 \pi^{2} \rho^{2} \mathrm{n}_{\text {clad }}}{L\left(U_{2}^{\infty 2}-U_{1}^{\infty 2}\right) \exp \left(-\frac{2}{V}\right)\left(1-\frac{2}{V}\right)}
$$

From Eqn. (4.2) to (4.4), the wavelength shift due to the change of external refractive index can be derived as

$$
\Delta \lambda=\lambda *\left(\exp \left(\frac{2}{V^{\prime}}-\frac{2}{V}\right)-1\right)
$$

where the revised normalized frequency $V^{\prime}$ follows

$$
V^{\prime}=\frac{2 \pi \rho}{\lambda+\Delta \lambda} \sqrt{\mathrm{n}_{\text {clad }}^{2}-\left(n_{\mathrm{ext}}+\Delta n_{\text {ext }}\right)^{2}} .
$$

It can be observed that the value $\left(2 / V^{\prime}-2 / V\right)$ increases as $n_{e x t}$ increases and $\rho$ decreases. In other words, the larger the external refractive index or the smaller the 
taper diameter, the higher is the sensitivity of the sensor. However, the decrease of the taper diameter is limited by the propagation loss induced by light scattering on the surface of the taper waist. Thus, optimization of the taper diameter is required. Due to the reduced effective refractive index in the waist region, the mode of light propagating through the microfiber is broadened. Fig. 4.1 shows the simulation results on the fraction of optical power exposing to surroundings with different taper diameters. In order to obtain effective sensing without significant propagation loss by light scattering, microfiber with a diameter of around $4 \mu \mathrm{m}$ is adopted in our experiments. The inset of Fig. 4.1 shows the simulated fundamental mode E-field profile of a microfiber with a diameter of $4 \mu \mathrm{m}$.

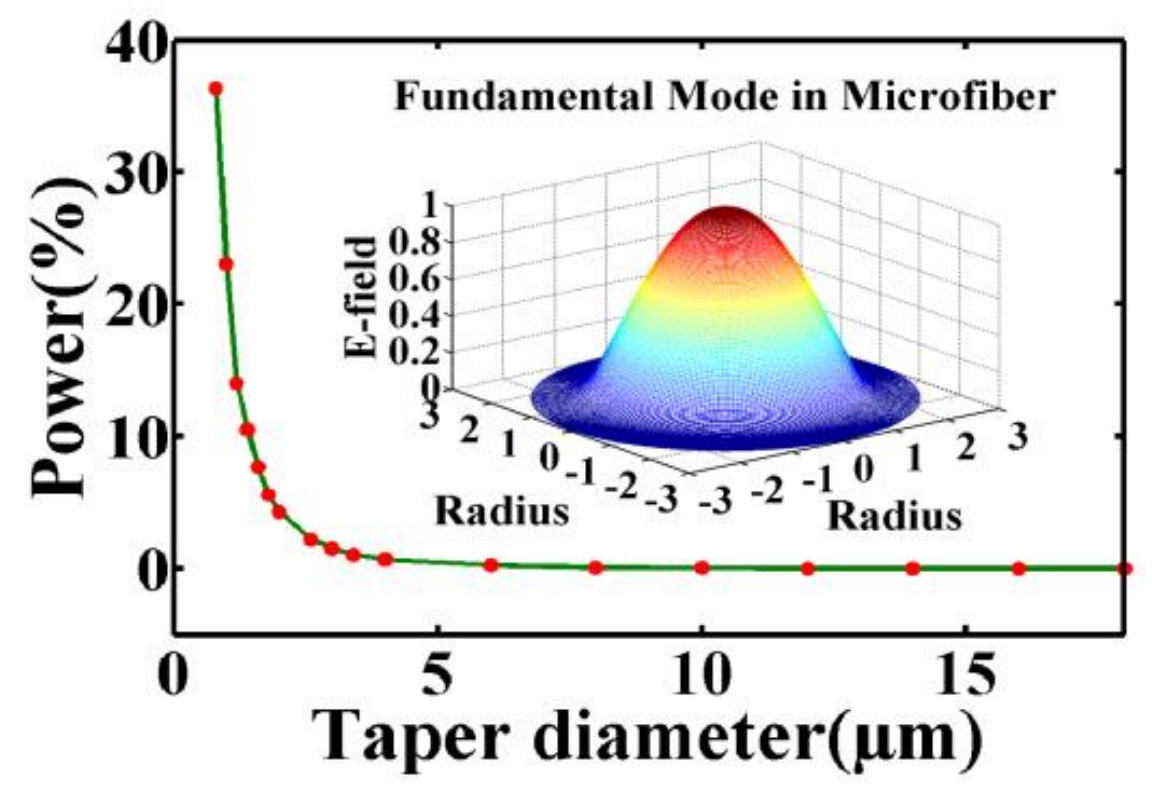

Figure 4.1 Plot of optical power coupling out of a microfiber against different taper diameter. The inset shows the E-field profile of fundamental mode of a microfiber with a taper waist diameter of $4 \mu \mathrm{m}$. 


\subsection{Experimental results}

The taper waist of the fabricated microfiber is generally uniform as it can be seen from the scanning electron microscope (SEM) image shown in Fig. 4.2(a). Since the unsymmetrical effect is common in tapering fiber as there is an initial tension to form the first transition, the tension to form the second transition will be much lower because the taper diameter has already been reduced. Therefore, the transition lengths are set differently to ensure symmetry of the taper, which are $1 \mathrm{~mm}$ in the left side and $5 \mathrm{~mm}$ in the right side. The experimental result of transition lengths viewed from the microscope built in the tapering machine are around $2.4 \mathrm{~mm}$ for the left and $1.6 \mathrm{~mm}$ for the right. The uniform waist length in our work is set as $5 \mathrm{~mm}$.

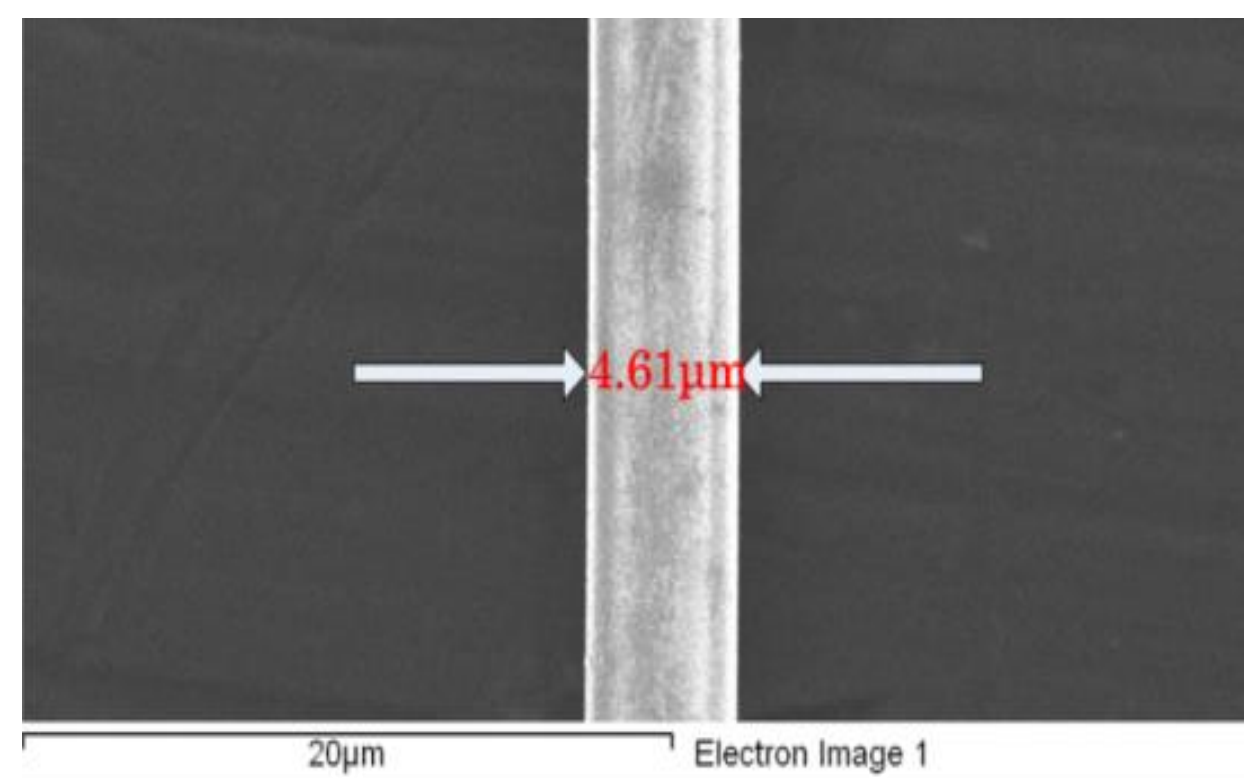

(a) 


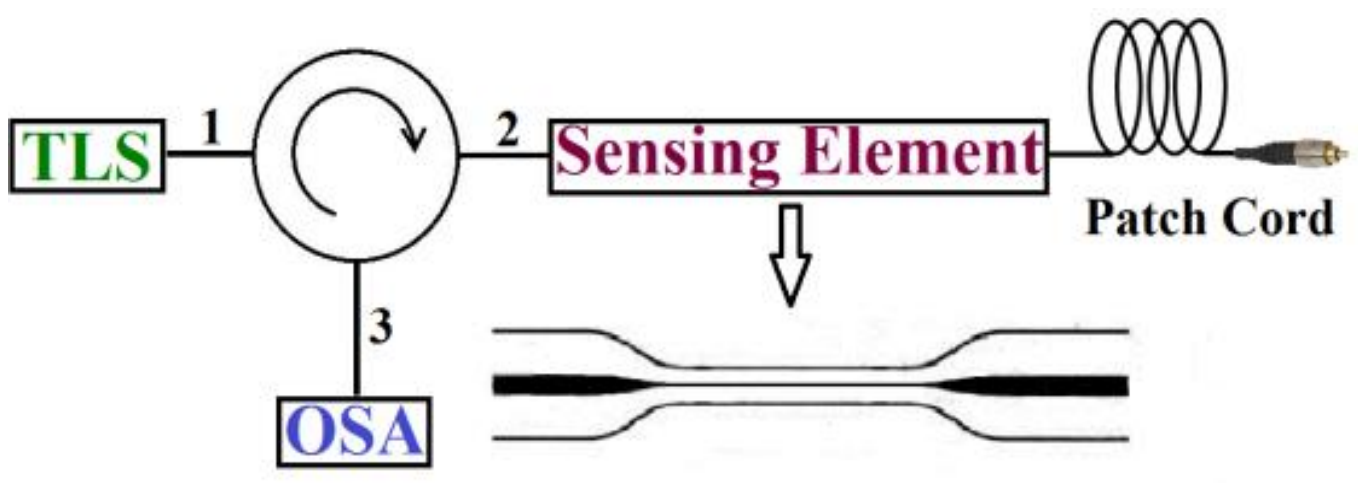

(b)

Figure 4.2 (a) SEM image of the taper waist with a diameter of $4.61 \mu \mathrm{m}$ and (b) experimental set-up of the refractive index sensor.

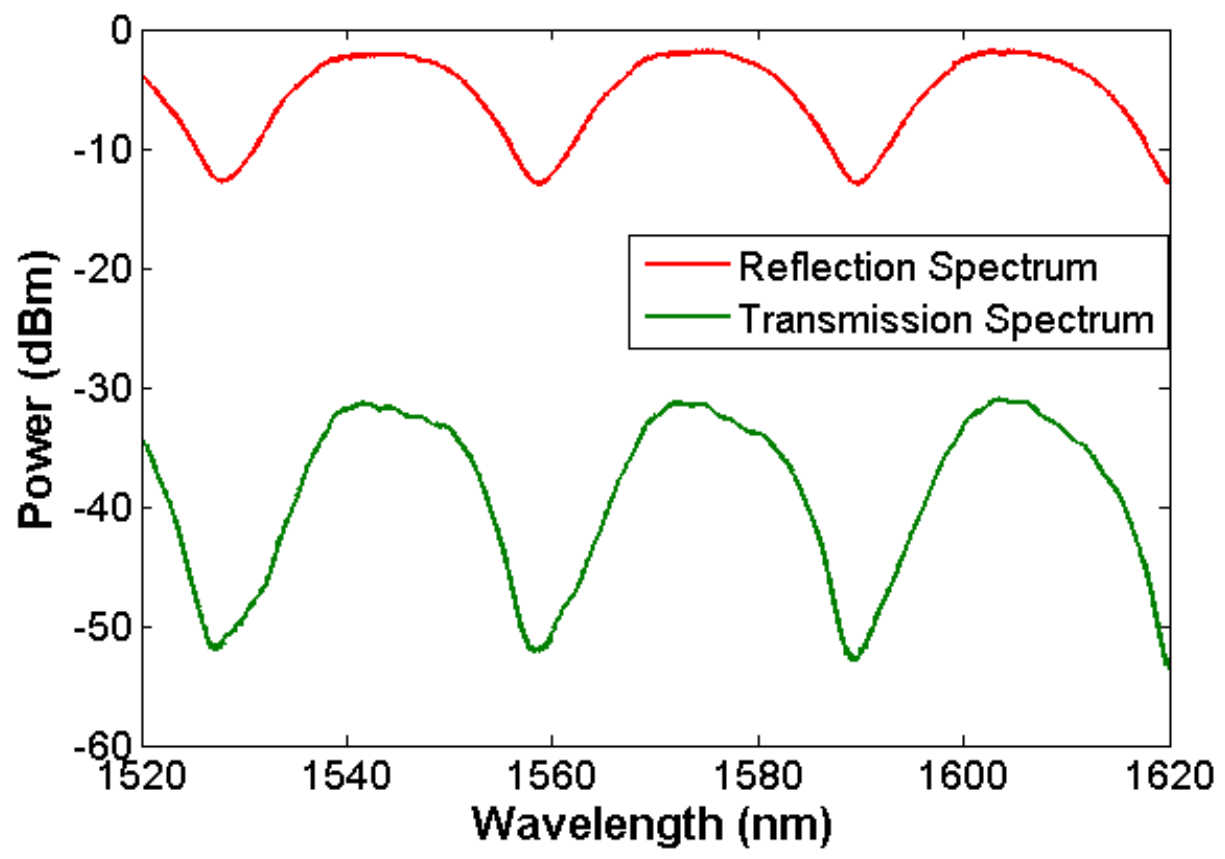

Figure 4.3 Reflection spectrum and transmission spectrum of a non-adiabatically tapered microfiber 


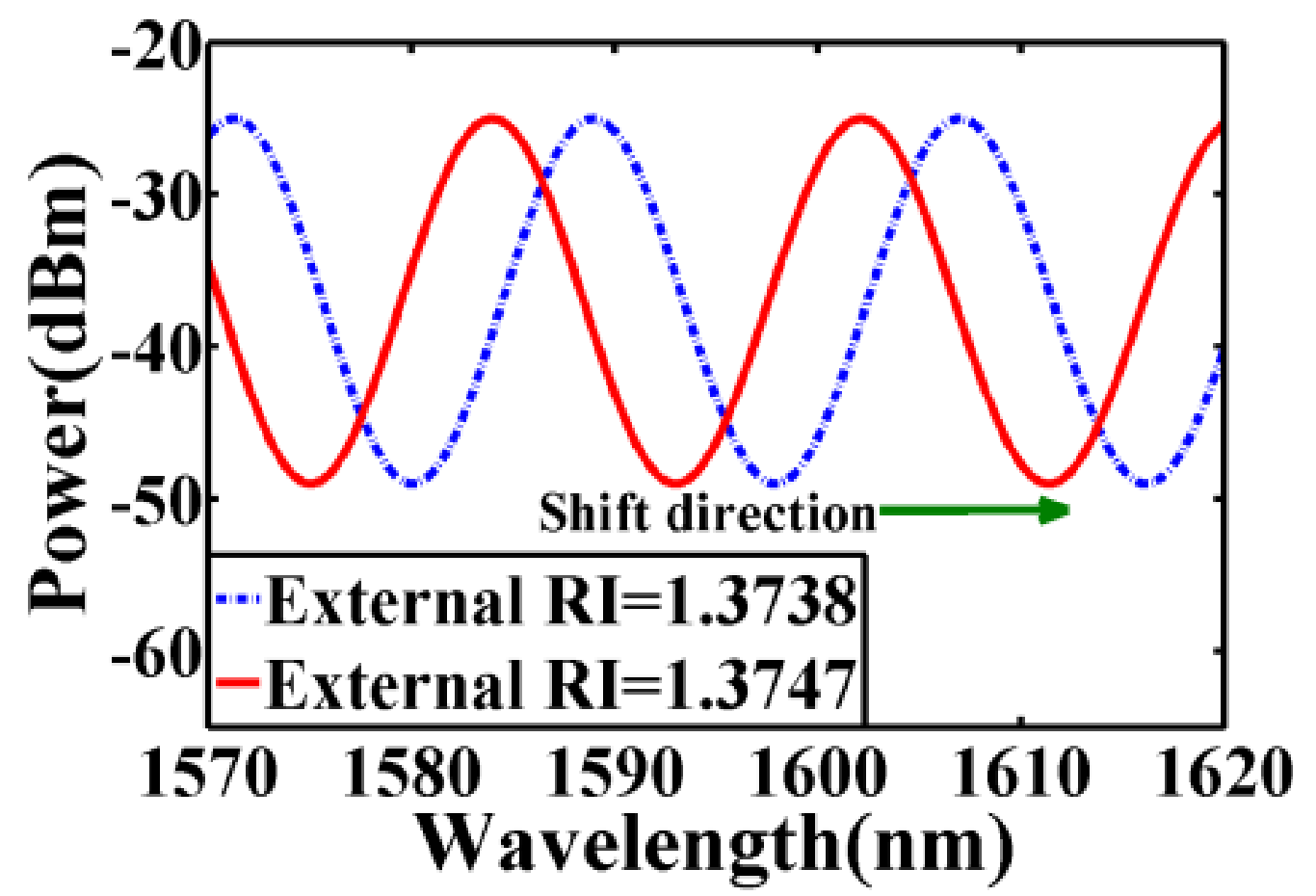

(a)

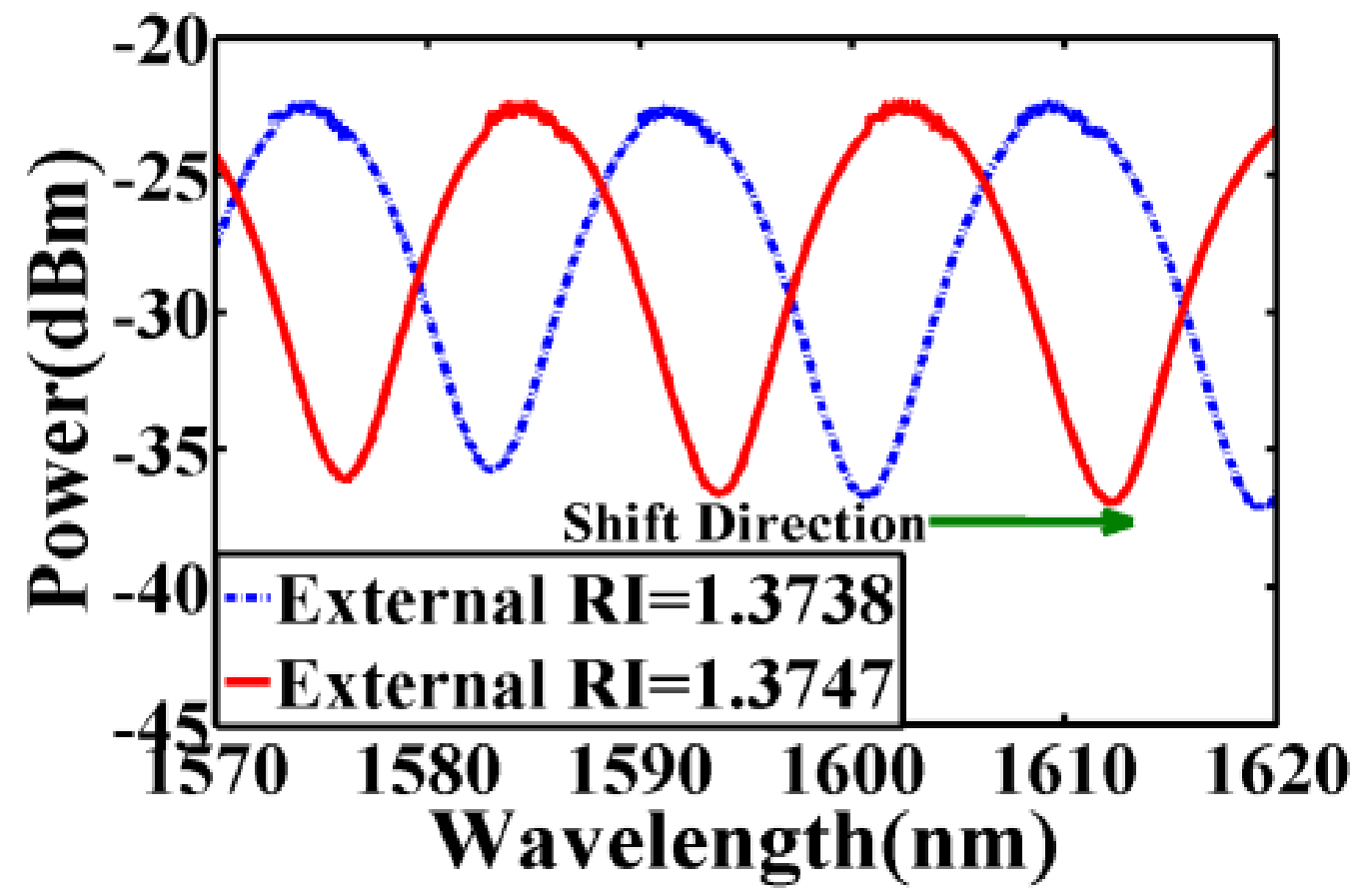

(b) 
Figure 4.4 (a) Calculated results and (b) experimental results of wavelength response of the microfiber sensor surrounded by different concentration of the salt solutions.

Fig. 4.2(b) shows the schematic diagram of the experimental setup of the microfiber sensor. Output light from a tunable laser source (Ando TLS AQ4321D, 1520-1620 nm) is coupled into port 1 of the circulator through port 2 towards the microfiber. The microfiber is surrounded by a few droplets of salt solution. Salt solutions with different refractive indices were prepared by diluting the saturated $\mathrm{NaCl}$ solution using distilled water. In all the author's work, the refractive index was measured by a refractometer (KEM RA-130) with a resolution of 0.0001 . Another end of fiber is terminated by a fiber patch cord. The effect of using the patch cord in this work is similar to coating a layer of gold on the end facet with the only difference being the lower reflectivity but more cost-effective. The reflected light from the microfiber is coupled via port 3 of the circulator to an optical spectrum analyzer (Ando OSA AQ6317B). The reason of monitoring the reflection spectrum instead of the transmission spectrum is similar to the double-pass in-line fiber taper MZI sensor [96]. It can be seen from Fig. 4.3 that the extinction ratio of the received power is larger in the reflection spectrum. The sharper resonance reduces wavelength reading errors.

From our experimental results (Fig. 4.4(b)), it was observed that an increase in external refractive index, a decrease in taper waist length, or an increase in wavelength will result in an increase in FSR which agrees with Eqn. (4.4) and Eqn. (4.5). More importantly, Fig. 4.4(a) and Fig. 4.4(b) depict the calculated results and the measured results of the wavelength response of the fabricated sensor respectively, which show good agreement with the 
simulated results. A tiny difference between the refractive index of the solution (around 0.0009 ) results in a resonance wavelength shift of over $10 \mathrm{~nm}$.

Fig. 4.5 illustrates the wavelength shift as a function of RI ranging from 1.33 to 1.37. Note that the wavelength shift from 0 to $200 \mathrm{~nm}$ is not obtained by referencing one single resonant dip. Instead, in each measurement the shift of the resonant wavelength that is closest to $1550 \mathrm{~nm}$ is recorded. The small measuring range of RI suggest a high sensitivity of the sensor, but is also a common drawback of such modal interferometers and MZI based sensors due to the periodic nature of the spectrum. The possible ways to improve this are by making the taper waist length shorter so that the FSR can be larger, or alternatively using asymmetrical taper such as the S shape taper [97] without compromising sensitivity. Fig. 4.6 shows the wavelength shift as a function of RI for two different fiber taper waist diameters, $4.61 \mu \mathrm{m}$ (square data points) and $5.11 \mu \mathrm{m}$ (circular data points). The fiber with smaller diameter shows a steeper slope suggesting that the fiber sensor has a higher sensitivity. In the RI range from 1.3707 to 1.3769 , the measured average sensitivity for sensor with a diameter of $4.61 \mu \mathrm{m}$ is $15532.24 \mathrm{~nm} / \mathrm{RIU}$ and the maximum sensitivity achieved is $18681.82 \mathrm{~nm} / \mathrm{RIU}$. With our OSA's resolution of $10 \mathrm{pm}$, the detection limit for RI is $5.35 \times$ $10^{-7}$. 


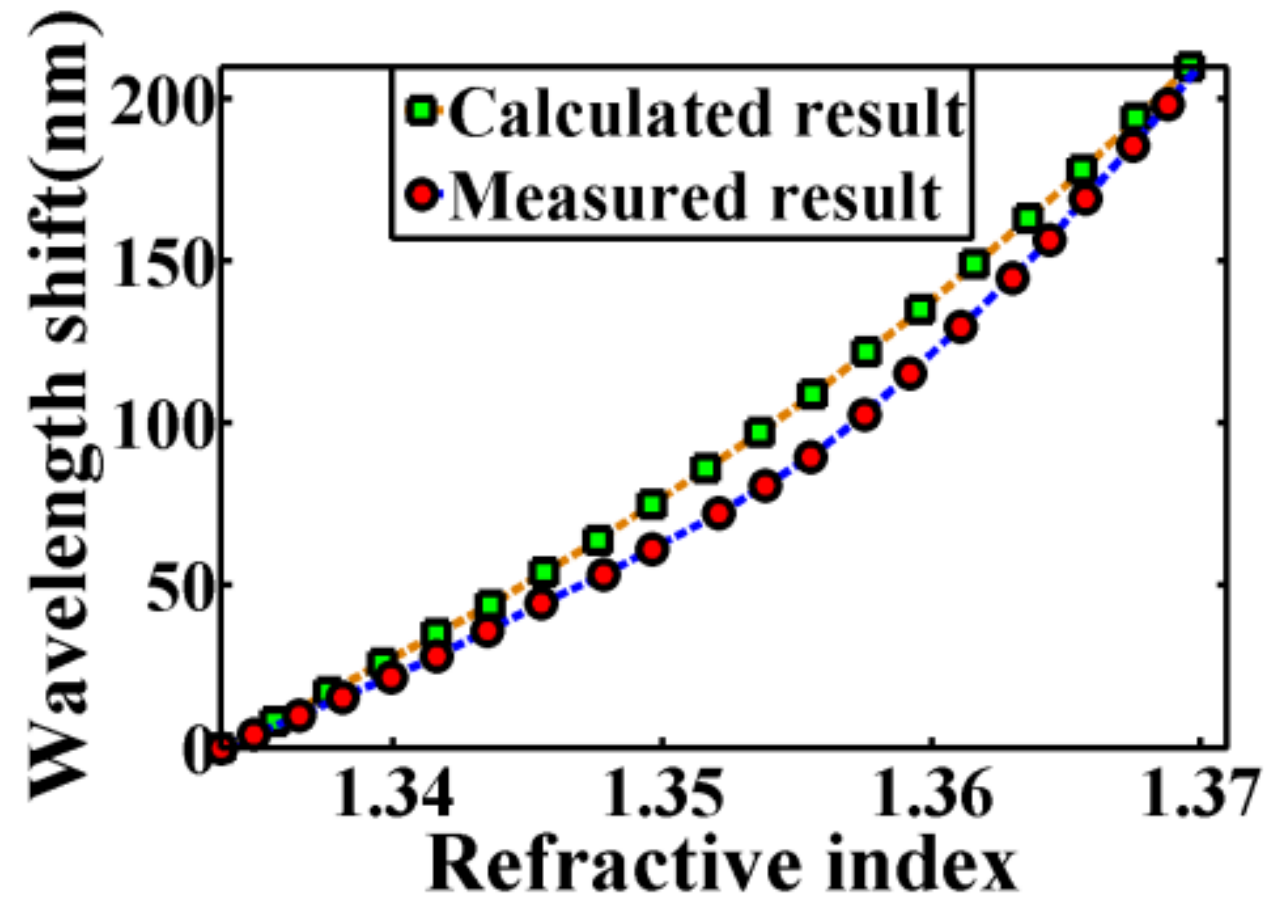

Figure 4.5 Resonant wavelength shift as a function of RI: theoretical prediction and measured result for waist diameter $\sim 5.11 \mu \mathrm{m}$.

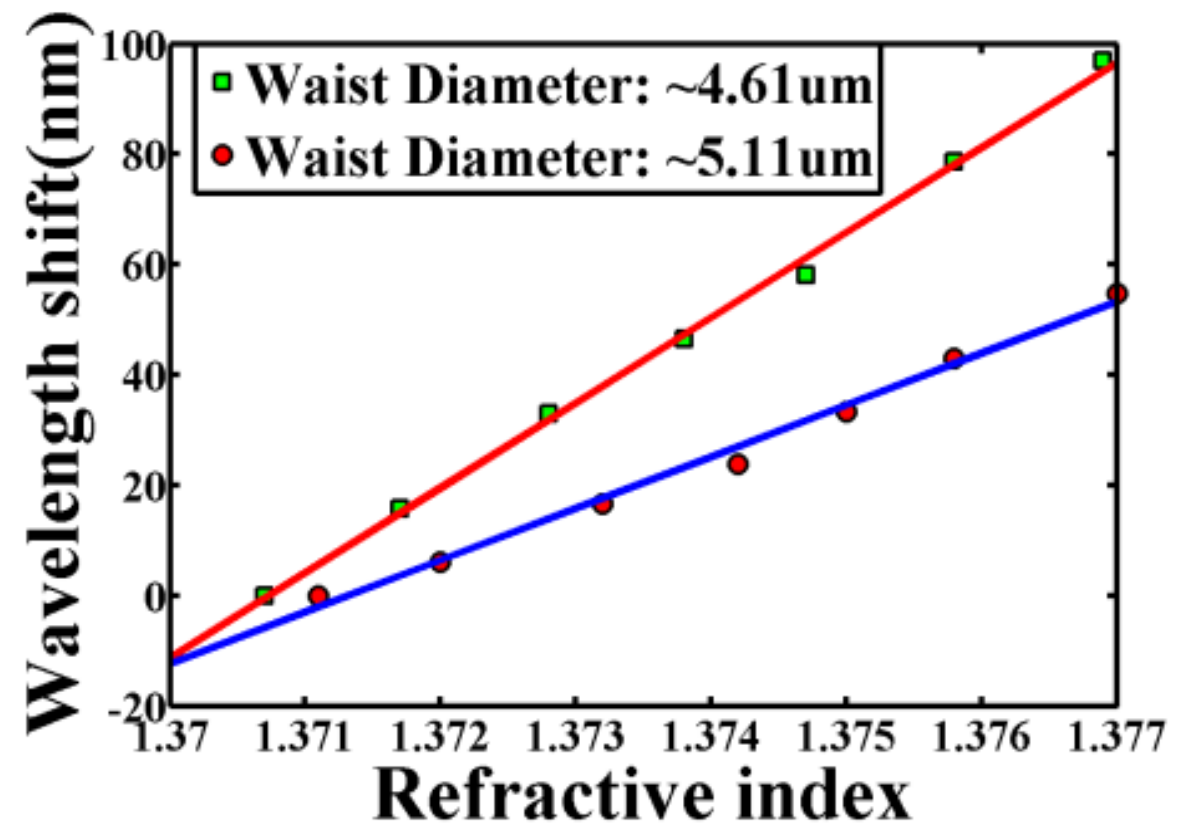


Figure 4.6 Resonant wavelength shift as a function of RI: comparison for two different taper diameters.

\subsection{Ultrahigh sensitivity}

As the theoretical analysis and preliminary results shown in the above section both indicated, the sensitivity of such abruptly tapered microfiber highly depends on the diameters of the taper waist. To further calibrate the relationship between the sensitivity and the diameter, a few more batches of microfibers were fabricated with different diameters. The tapering parameters such as the temperature of the graphite filament, pulling speed, and the power degradation rate were set to be exactly the same for all these microfibers. Moreover, the taper profiles, including the taper transition lengths and the taper waist lengths, were all preset to be the same except for the diameters. As shown in Fig. 4.7 to Fig. 4.9, these three examples of microfibers were tested using glycerol solutions with RI=1.4064 and 1.4068. The resonant wavelength shift for the one with diameter of $9.5 \mu \mathrm{m}$ is around 3.5 $\mathrm{nm}$ and the sensitivity is calculated to be $8750 \mathrm{~nm} / \mathrm{RIU}$. The wavelength shift for the one with diameter of $4.8 \mu \mathrm{m}$ reaches $22 \mathrm{~nm}$ and the sensitivity is calculated to be $55000 \mathrm{~nm} / \mathrm{RIU}$. When the fiber diameter was further reduced to $3.9 \mu \mathrm{m}$, the wavelength shifts over $26 \mathrm{~nm}$ and the sensitivity reaches $65000 \mathrm{~nm} / \mathrm{RIU}$ which is higher than nearly most of the optical fiber sensors that have been reported so far. 


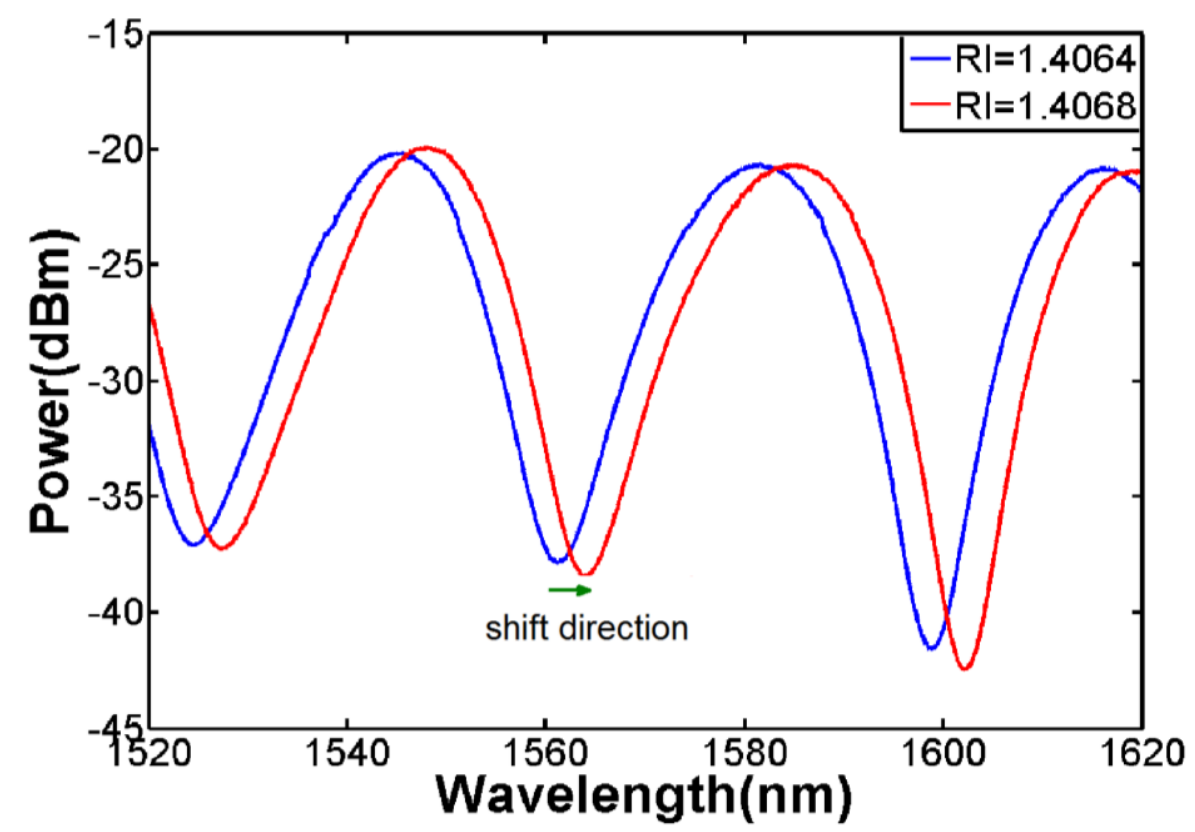

Figure 4.7 Wavelength responses for microfiber with diameter of $9.5 \mu \mathrm{m}$ in glycerol solutions with RI of 1.4064 and 1.4068

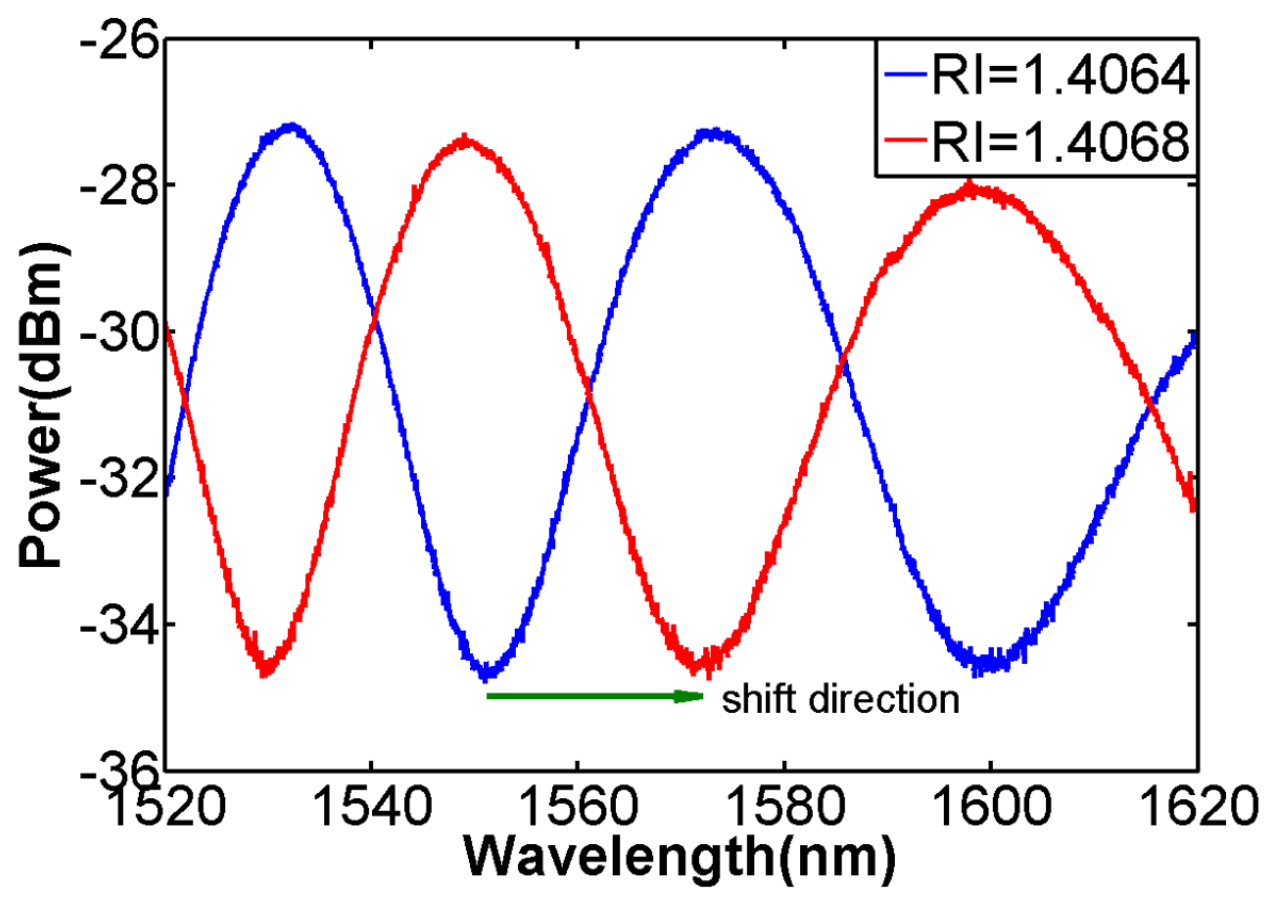


Figure 4.8 Wavelength responses for microfiber with diameter of $4.8 \mu \mathrm{m}$ in glycerol solutions with RI of 1.4064 and 1.4068

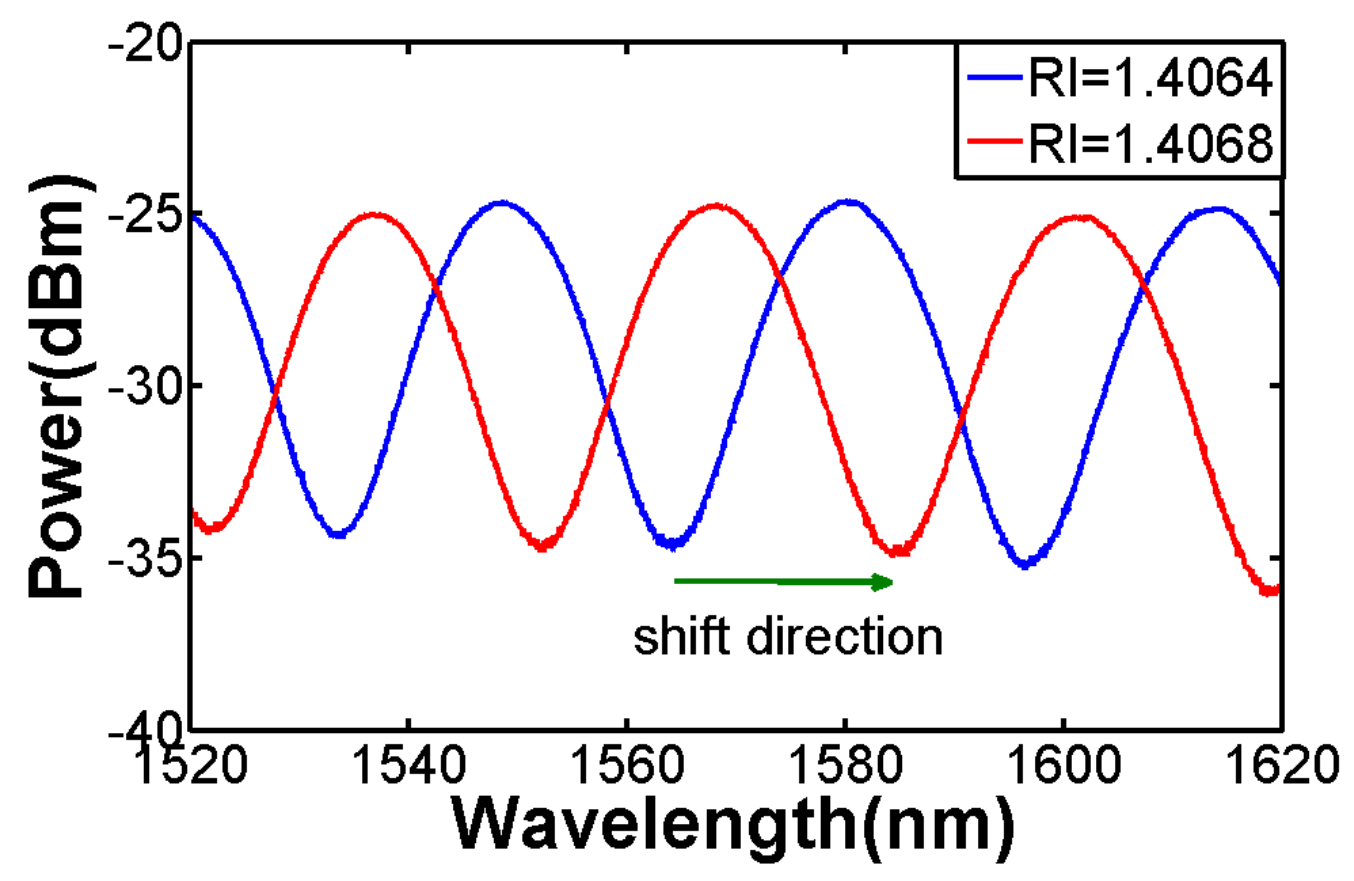

Figure 4.9 Wavelength responses for microfiber with diameter of $3.9 \mu \mathrm{m}$ in glycerol solutions with RI of 1.4064 and 1.4068 


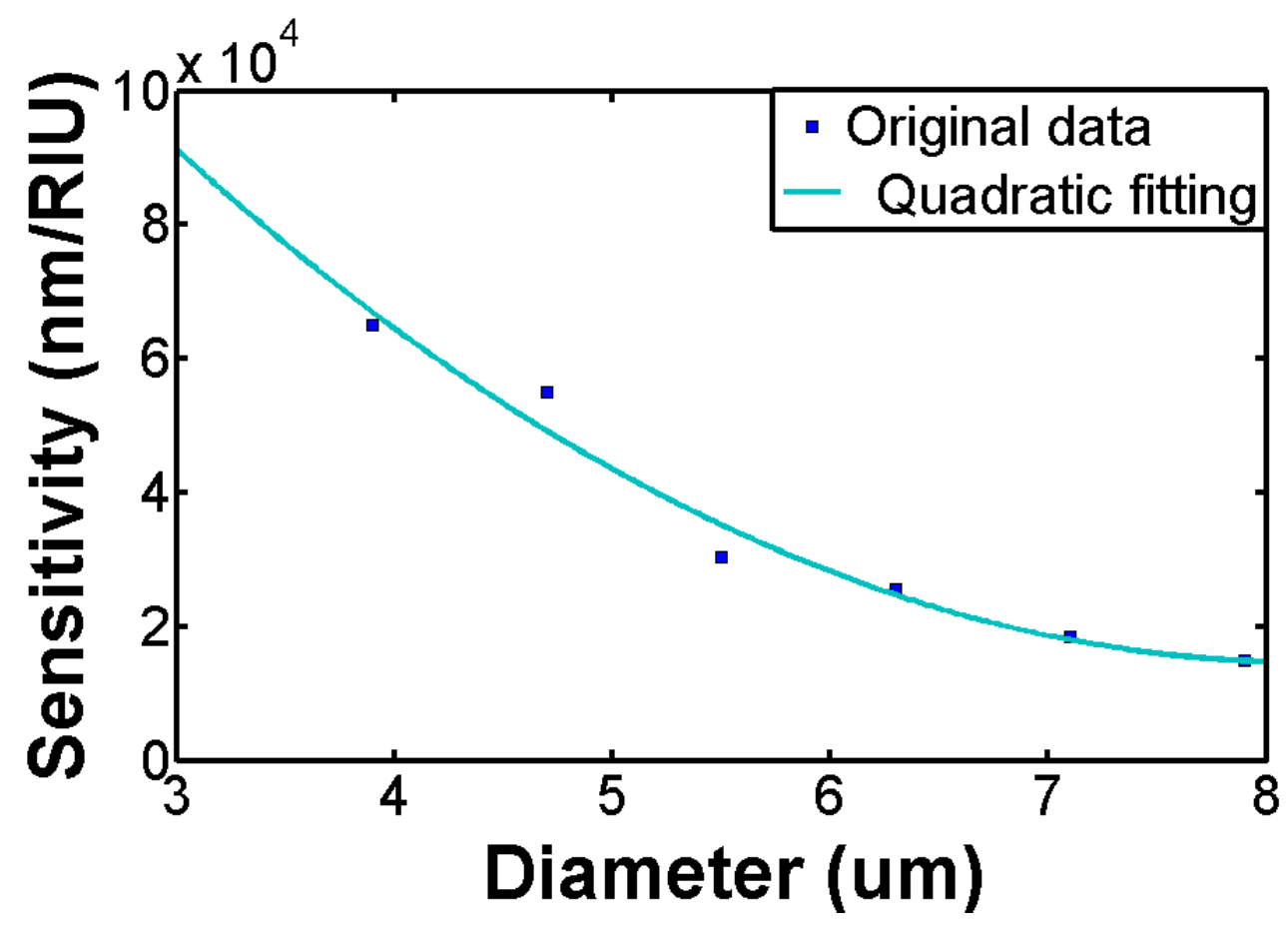

Figure 4.10 Sensitivity characterization with different diameters and a quadratic fitting

Fig. 4.10 illustrates the relationship between the sensitivity and the diameter. It can be clearly seen that the diameter of the microfiber is inversely proportional to the sensitivity of the sensor. As we reduce the diameter further down to below $3 \mu \mathrm{m}$, the effective refractive index of the fundamental mode becomes very low compared to the RI of silica. The effective refractive index of the fundamental mode is associated with the RI of the silica fiber, RI of the ambient medium, the beat length, and the percentage of the power leaking out. Once we drip the glycerol solution with RI of 1.4 to the sensing region, the output spectrum showed a flat response suggesting a high loss in the reflected spectrum. This indicates that the mode distribution changes in a way that the fundamental core mode is completely coupled into the surrounding medium since its effective refractive index becomes much lower than the glycerol solution. Hence there is no more coupling effect in the microfiber and no more sinusoidal spectrum observed in the OSA. 


\subsection{Dual parameter sensing}

As FBG and abruptly tapered microfiber can both be characterized by looking at the reflection spectrum, it would be natural to combine these two and make a sensor that can sense not only refractive index but also strain and temperature.

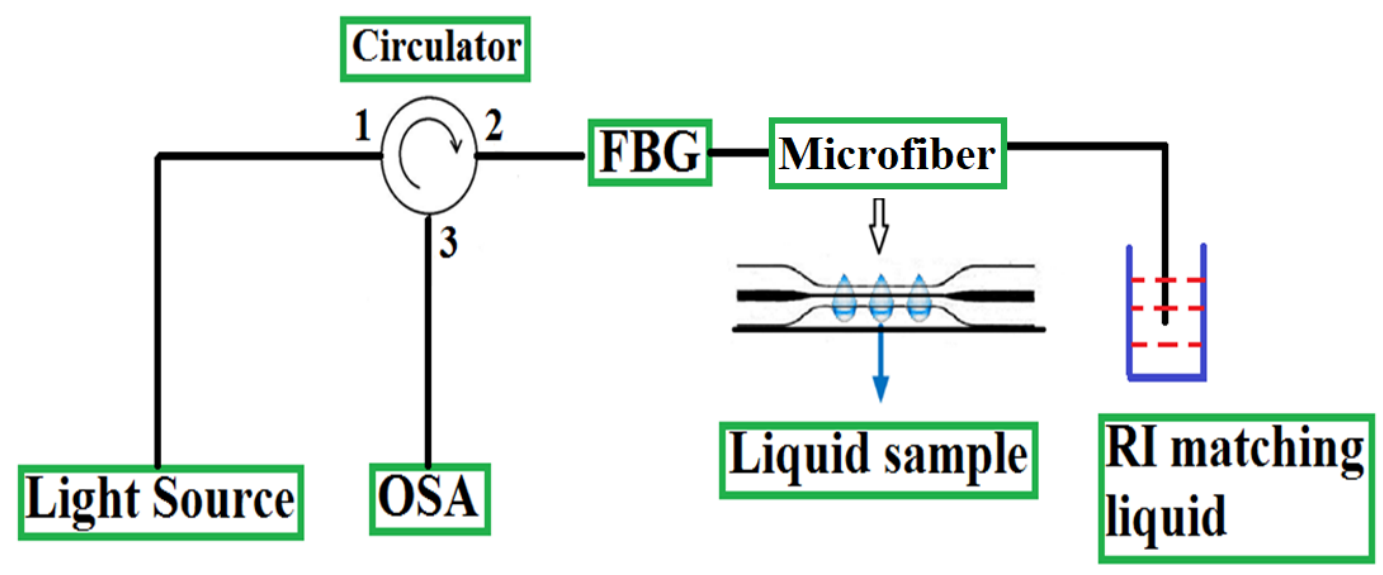

Figure 4.11 Setup of combining FBG and abruptly tapered microfiber as dual parameter sensor

The setup is shown in Fig. 4.11 in which the configuration of examining reflection spectrum is the same with the one shown in previous sections. The FBG and microfiber were cascaded in series with the FBG before the microfiber in the transmission direction as shown in the figure. This ensures that the Bragg wavelength of the FBG is reflected before the light is transmitted through the microfiber. Therefore, the peak intensity of the Bragg wavelength will not be affected by variations in the microfiber sensor region. The light then propagates through the microfiber and reaches the end facet of fiber. The fiber end facet was cleaved and placed into an index matching liquid with RI of around 1.45. In this case, the light will be totally transmitted into the liquid, as can be seen by the Fresnel equations of reflection $[98,99]$. Reflection at the end facet occurs when the refractive index of the 
surrounding medium is different from the index of the fiber core. The fraction of the incident power that is reflected at the interface is given by the reflectance or reflectivity $R$, and the fraction that is refracted is given by the transmittance or transmissivity $T$ [98]. For nonmagnetic mediums, the reflectance of $s$-polarized light is given by

$$
R_{s}=\left|\frac{n_{1} \cos \theta_{i}-n_{2} \cos \theta_{t}}{n_{1} \cos \theta_{i}+n_{2} \cos \theta_{t}}\right|^{2},
$$

while the reflectance for $p$-polarized light becomes

$$
R_{p}=\left|\frac{n_{1} \cos \theta_{t}-n_{2} \cos \theta_{i}}{n_{1} \cos \theta_{t}+n_{2} \cos \theta_{i}}\right|^{2} .
$$

$n_{1}$ and $n_{2}$ are the refractive indices of the two mediums. $\theta_{i}$ is the incident angle and $\theta_{t}$ is the transmission angle. As a consequence of the conservation of energy, the transmittances are given by $T_{s}=1-R_{s}$ and $T_{p}=1-R_{p}$. If the incident light is unpolarized (containing an equal mix of $s$ - and $p$-polarizations), the reflectance is

$$
R=\frac{R_{s}+R_{p}}{2}
$$

In our case, since the fiber's end is properly cleaved, the incident angle of the light coming from the silica fiber can be considered to be 0 because the light path in the fiber core is nearly parallel with the propagation axis of the fiber. Hence the reflection and refraction angles are also 0 . If the RI of the liquid is matched to that of the silica fiber core, the reflectance would be 0 according to Eqs. (4.8) to (4.10). In this case, port 3 of the circulator will receive nothing but the Bragg wavelength. This is verified in Fig. 4.12 and it functions as a FBG sensor. In practice however, one can see that there still exist some coupling effect 
since the wavelength response between 1520 and $1620 \mathrm{~nm}$ is still sinusoid. This is because of the fact that it is almost impossible to perfectly match the RIs of the fiber core and the liquid leading to a non-zero reflectance at the interface between the cleaved surface and the liquid. More importantly, the low amplitude of the sinusoidal wavelength response does not have any impact on the FBG sensor for sensing either temperature or strain.

On the other hand, when the fiber end is taken out of the RI matching liquid, the fiber end facet now acts as a mirror just like connecting a patch cord shown in the previous sections. This is another reason for why the fiber end needs to be well cleaved. Fig. 4.13 shows the spectrum of a non-adiabatically tapered microfiber sensor with a FBG peak at $1560 \mathrm{~nm}$. This FBG peak will also have no influence to the refractive index sensing in microfiber region at all.

In summary, due to the same configuration of the characterization system for FBG and non-adiabatic microfiber sensor, we have investigated the combination of these two sensors into one system and the result is promising. Such design could not only multiplex many sensors into one system but also is capable of sensing different targets such as refractive index, temperature and strain independently. 


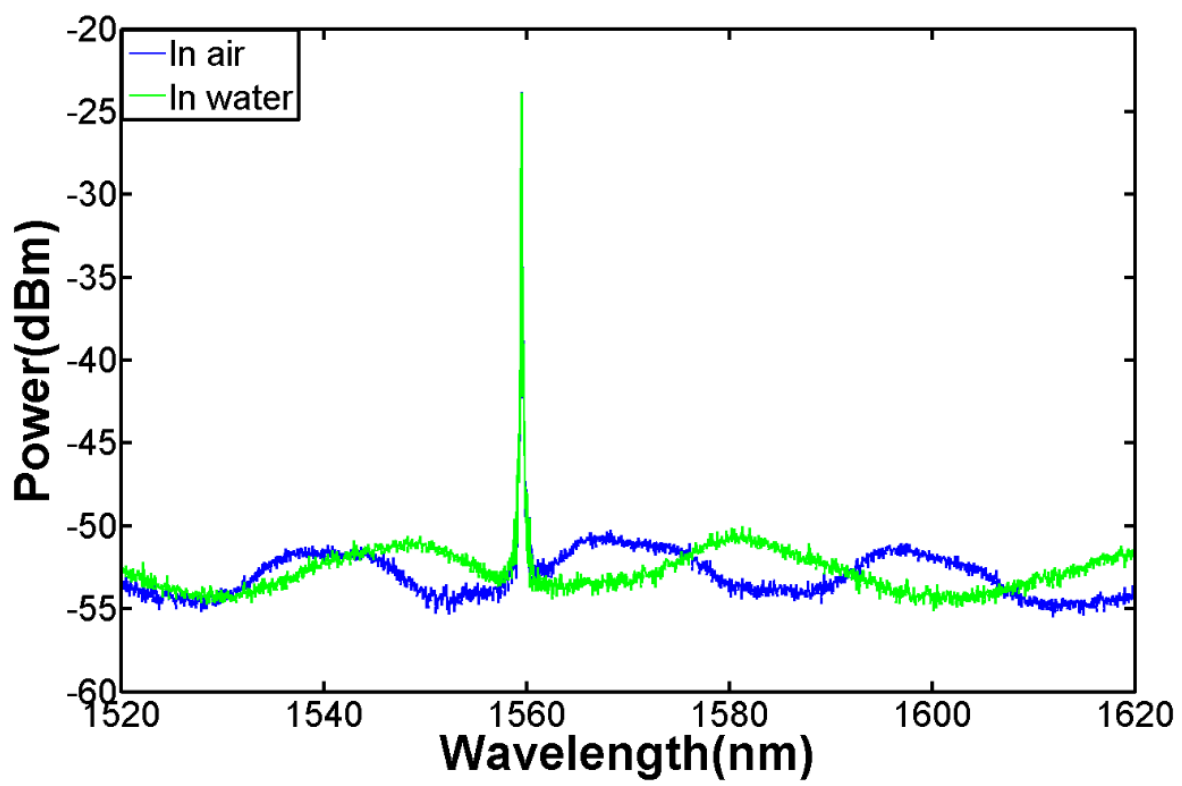

Figure 4.12 A FBG sensor. The refractive index change in the surrounding area of the microfiber does not have any impact. 


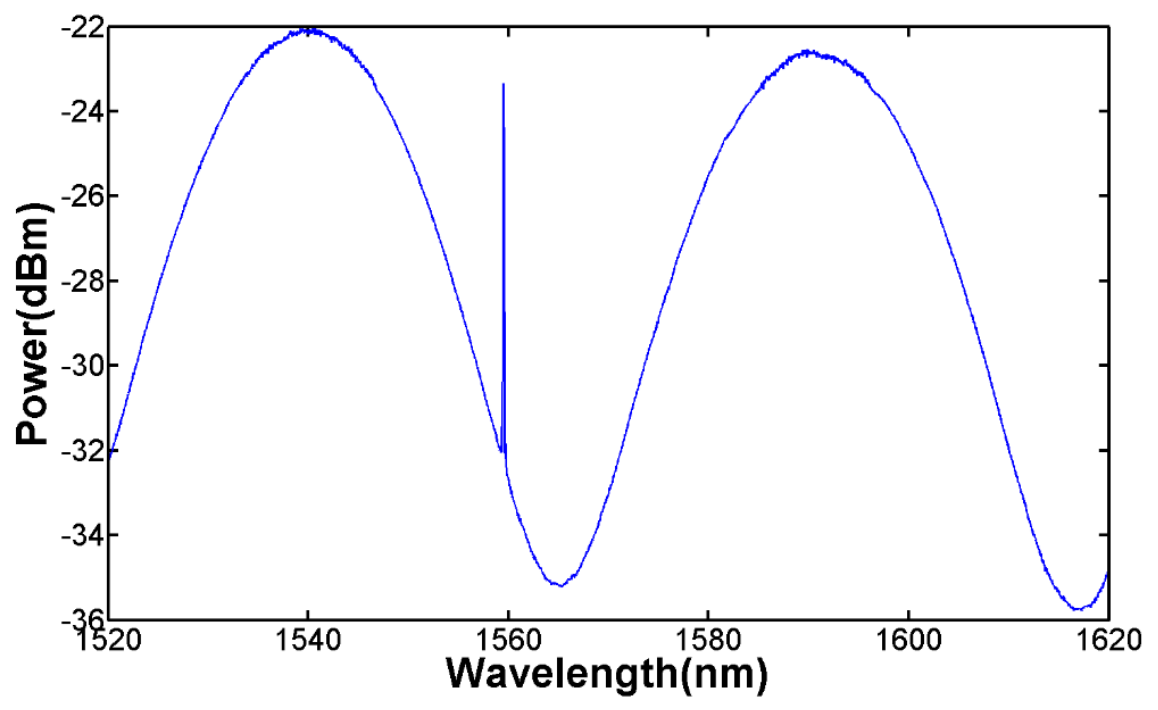

Figure 4.13 A refractive index sensor spectrum. The FBG peak will not have impact. 


\section{Chapter 5 Optimization of non-adiabatic microfiber sensor}

\subsection{Drawback of non-adiabatic microfiber sensor}

For most of these interferometer-based sensors, there is one common drawback in terms of the measuring range for the refractive index. As the wavelength responses of these modal interferometers is periodic, once the wavelength shifts, due to the change of external refractive index, exceeds the free spectral range (FSR), it is impossible to determine how much the resonant wavelength has shifted. Hence the sensitivity of such sensors cannot be estimated [39]. Fig. 5.1 is an illustration of how the FSR affects the measurement of wavelength shift. The FSR of this microfiber sensor is only $20 \mathrm{~nm}$. The blue spectrum corresponds to RI of 1.3738 and it shifts $15 \mathrm{~nm}$ towards right when the external RI changes to 1.3769 , as the red spectrum depicts. One can see that if the external RI has a larger change, the blue plot will shift beyond one cycle and the exact value could not be determined. 


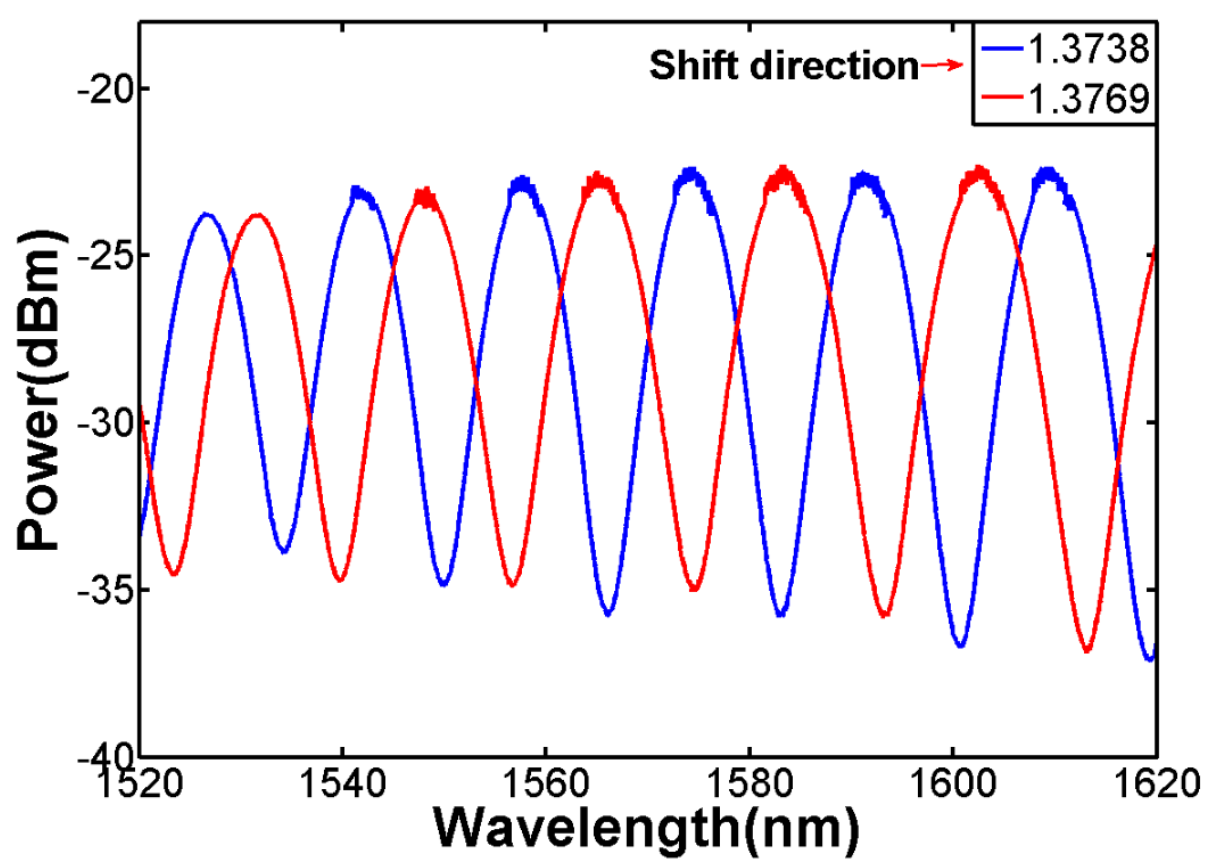

Figure 5.1 The microfiber sensor with FSR of only $20 \mathrm{~nm}$

To address this issue, it is necessary to extend the free spectral range of the interference pattern. In Chapter 4, we have derived the relationship between FSR and fiber dimensions. Recall Eqn. 4.5, one can see that FSR is proportional to square of microfiber's radius and inversely proportional to the microfiber's waist length. Since the radius of the microfiber plays a key role in determining the sensitivity of this sensor, we need the radius to be as small as possible if high sensitivity is to be achieved. This is because the smaller radius of the microfiber will result in a stronger evanescent field that interacts with the surrounding of the microfiber. In this case, the effective refractive index of the relevant modes will more likely to be influenced by the external refractive index. This suggests that a microfiber of smaller radius would have a greater sensitivity to the refractive index in its immediate surrounding [39]. Therefore, reducing the waist length is the most feasible way of achieving 
a larger FSR while maintaining a relatively high sensitivity to refractive index variations in the surrounding environment.

\subsection{Short taper}

So far, the author has demonstrated the non-adiabatic fiber taper which has extraordinary sensitivity to refractive index variation of the ambient medium. Nevertheless, the drawback and corresponding solution were both pointed out in last section. The goal is to fabricate microfibers with short taper waist without compromising too much on the sensitivity. Using a fusion splicer is one simple way to obtain microfiber probes with very small waist lengths since the width of the arc generated by the electric poles is narrow [100,101]. However, this fabrication technique will result in a decrease in the sensitivity since the thinnest part of the waist may be too short, making it difficult to control the waist length of the microfiber. In this work, a commercial microfiber fabrication machine (Vytran, GPX-3000) with motorized fiber holding stages was used.

Fig. 5.2 shows the spectrum of an abrupt taper with short taper ( set at $2 \mathrm{~mm}$ ) waist that we have fabricated. It can be seen that the FSR now extends over $60 \mathrm{~nm}$. Compared to the work in the previous section whose waist length was set to be $5 \mathrm{~mm}$, the FSR is increased by about 4 times. This greatly improves the measuring range of RI. 


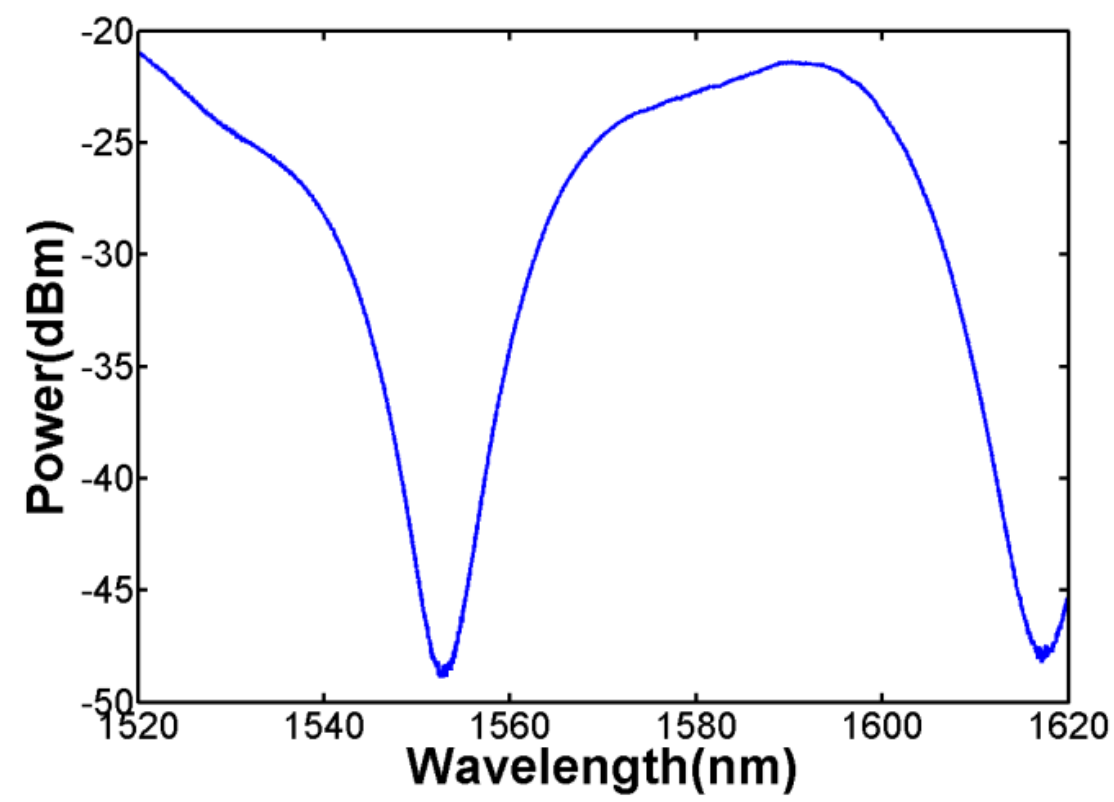

Figure 5.2 Wavelength spectrum of abrupt fiber taper with short waist. The taper profile was set as $1 \mathrm{~mm}$ for upper taper, $2 \mathrm{~mm}$ for waist length, $2 \mathrm{~mm}$ for down taper. The diameters were set as $5 \mu \mathrm{m}$.

However, the sensitivity was compromised. The reason behind this is that the waist diameter in this case is actually much larger than the one we set in the software. This is intuitive since it will be considerably difficult to taper the fiber to a small diameter while at the same time making the central waist extremely short. If we look back to Eqs. (4.6) and (4.7), we may easily find that the sensitivity of such non-adiabatically tapered microfiber sensor highly depends on the diameter of the waist. The larger diameter will lead to a lower sensitivity.

Therefore, several possible ways of making the taper waist short but thin were carried out. It was found that the most effective way is to reduce the pulling speed of the fiber hold block. As the pulling speed becomes slow, the taper waist part will spend more time going 
through the filament of the tapering machine so that the waist diameter can be potentially smaller. The results were compared as shown in Fig. 5.3. In both the two cases, the taper profiles in terms of upper taper length, waist length, down taper length and waist diameter, were set to be exactly the same. The only difference is that the pulling speed in Fig. 5.3 (a) is $0.5 \mathrm{~mm} / \mathrm{s}$ and the one in Fig. 5.3 (b) is $0.2 \mathrm{~mm} / \mathrm{s}$. One can easily calculate the sensitivities in RI range of 1.377 to 1.38 , which are $3500 \mathrm{~nm} / \mathrm{RIU}$ for (a) and $4500 \mathrm{~nm} / \mathrm{RIU}$ in (b). Thus, it is clear that the diameter of the taper can actually be reduced by adopting a slower pulling speed, hence resulting in a larger sensitivity of the short-taper microfiber sensor.

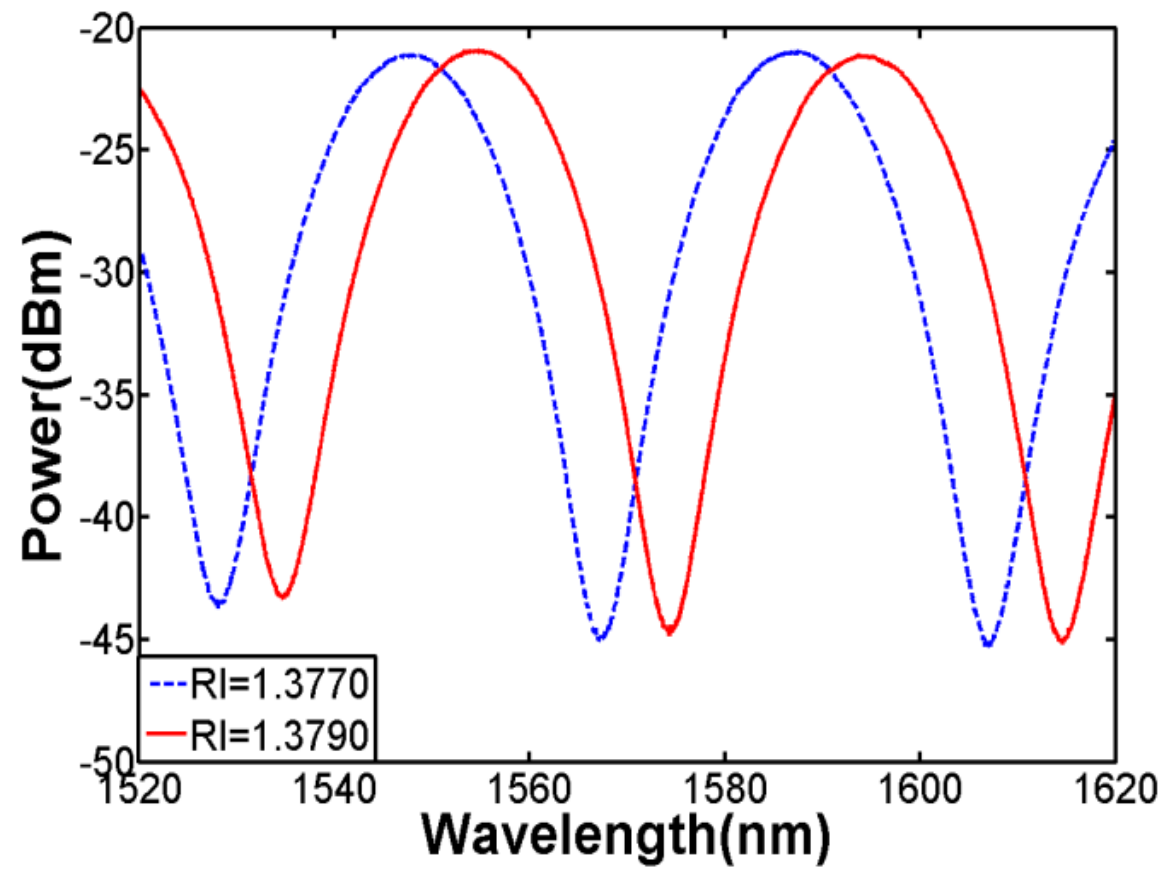

(a) 


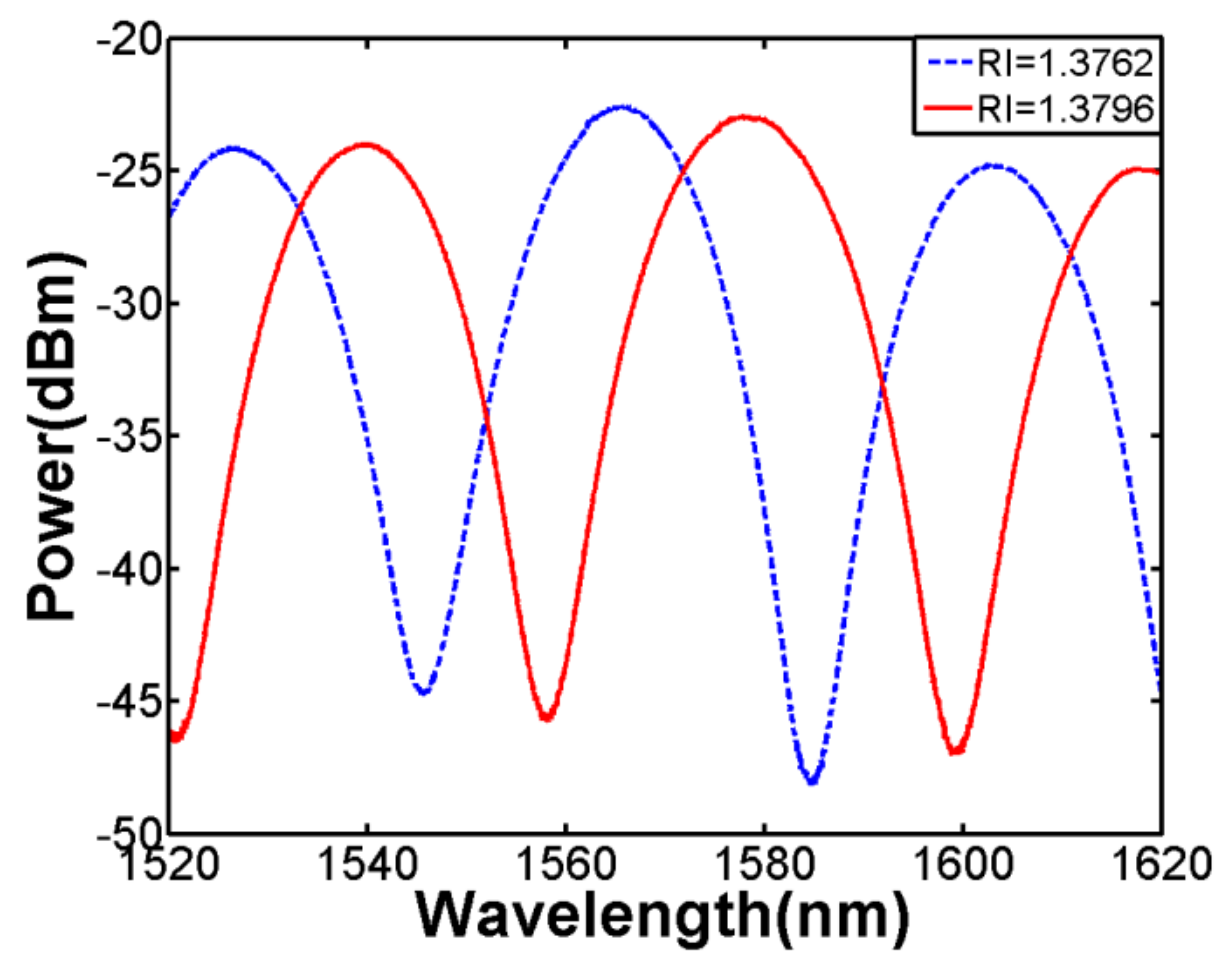

(b)

Figure 5.3 Wavelength response of abrupt taper with pulling speed of (a) $0.5 \mathrm{~mm} / \mathrm{s}$ (b) 0.2 $\mathrm{mm} / \mathrm{s}$, both the two taper's profiles were set as $1 \mathrm{~mm}$ for upper taper, $3 \mathrm{~mm}$ for waist length, $2 \mathrm{~mm}$ for down taper. The diameters were both set as $5 \mu \mathrm{m}$.

\subsection{Characterization and discussion}

Knowing the volume conservation of the fiber during the tapering process, fabricating a short microfiber while ensuring that the radius is as small as possible would pose a considerable challenge. The trade-off between the diameter and the waist length of the microfiber is the main issue in this work. The condition for such trade-off is not easy to be quantified as the minimum waist length of a fiber taper is limited by the width of the heating source and the widths in various tapering systems are different. 
In a fusion splicer tapering system, the fiber could be tapered into micrometer scale, yet with very short waist length [101]. This is due to the fact that the width of the electric arc generated by the electric poles is quite small, normally within hundreds of micrometers. However, it is not possible to control the length of the fiber taper in such a tapering system as it has no programmable motor stages. Moreover, the accumulated phase difference between the two modes implied in Eq. (4.2) and (4.3) will be small due to the short beating region of such fiber taper which elongates to only a few hundreds of micrometer. The sensitivity in the work of Du et al [101] is significantly lower than the result demonstrated in Ref. [39]. Therefore, these are two main drawbacks of the fusion splicer tapering system.

As the minimum waist length of a fiber taper is limited by the width of the heating source, the aim of this work is to produce a microfiber with the waist length approaching the limit, and make the diameter to be as small as possible at the same time. The author has successfully fabricated such a microfiber with waist length of $2.4 \mathrm{~mm}$ which is almost close to the width of the heating source ( $2 \mathrm{~mm}$ width graphite filament in our system) and with diameter of around $7 \mu \mathrm{m}$.

The most dominant parameters to form a desired fiber taper are the heating power and the pulling speed. The tension monitor of the microfiber fabrication machine is used to calibrate the normalized power of the graphite filament as well as its respective power degradation rate for microfiber fabrication. The power is close to the normalized value when the fiber tension detected by the monitor during the fabrication process is maintained at a certain positive level with slight periodic fluctuations. In this work, the normalized power of the filament was calibrated as $48 \mathrm{~W}$ and the power degradation rate is $-8 \%$ during the waist 
drawing period. The physical meaning of the normalized power is that the fiber will be melted in a temperature that is neither too cool nor too hot, which was calibrated by the fiber's tension curve of the tension monitor. Table 1 shows the relationship between the fabrication parameters and the resulting microfiber profiles. The temperatures generated by the three sets of power levels of the filament are all around the normalized value. It is clearly seen that the minimum diameter we can achieve is likely to be small once the filament power (47 W) is set slightly below the normalized power (48 W). Furthermore, the minimum diameter can be further reduced by increasing the pulling speed. 


\begin{tabular}{|c|c|c|c|c|}
\hline \multicolumn{5}{|c|}{$\begin{array}{l}\text { PS-pulling speed }(\mathrm{mm} / \mathrm{s}), \text { WL-waist length }(\mathrm{mm}), \text { MD-repeatable minimum diameter } \\
\qquad \operatorname{achieved}(\mu \mathrm{m}), \text { MS-maximum sensitivity }(\mathrm{nm} / \mathrm{RIU})\end{array}$} \\
\hline & \multicolumn{2}{|r|}{ PS: 0.5} & \multicolumn{2}{|c|}{ PS: 1} \\
\hline & WL: 2.4 & WL: 4.8 & WL: $\sim 2.4$ & WL: $\sim 4.8$ \\
\hline $47 \mathrm{~W}$ & $\begin{array}{l}\text { MD: } \sim 8.1 \\
\text { MS: } \sim 13,000\end{array}$ & $\begin{array}{l}\text { MD: 4.9 } \\
\text { MS: } \sim 50,000\end{array}$ & $\begin{array}{l}\text { MD: } ~ 7.1 \\
\text { MS: 25,000 }\end{array}$ & $\begin{array}{l}\text { MD: 4.7 } \\
\text { MS: } 52,000\end{array}$ \\
\hline $48 \mathrm{~W}$ & $\begin{array}{l}\text { MD: 8.6 } \\
\text { MS: } \sim 10,000\end{array}$ & $\begin{array}{l}\text { MD: 5.2 } \\
\text { MS: 48,000 }\end{array}$ & $\begin{array}{l}\text { MD: 7.4 } \\
\text { MS: 19,000 }\end{array}$ & $\begin{array}{l}\text { MD: 5.0 } \\
\text { MS: 50,000 }\end{array}$ \\
\hline $49 W$ & $\begin{array}{l}\text { MD: 8.5 } \\
\text { MS: } 10,000\end{array}$ & $\begin{array}{l}\text { MD: 5.5 } \\
\text { MS: 45,000 }\end{array}$ & $\begin{array}{l}\text { MD: 8.6 } \\
\text { MS: 10,000 }\end{array}$ & $\begin{array}{l}\text { MD: 5.4 } \\
\text { MS: 45,000 }\end{array}$ \\
\hline
\end{tabular}

Table 1. Characterization of the filament power and pulling speed with the waist length and waist diameter of the microfiber.

The experimental set-up as depicted in Fig. 5.4 is a double-pass configuration or reflection-spectrum configuration. The light from the light source will propagate through the microfiber and be reflected by the end facet of the patch cord. The fibers the author used were all normal single-mode fibers. The reflected light will then propagate through the microfiber one more time before being coupled into the optical spectrum analyzer (OSA) by the circulator. Thus, such a set-up increases the extinction ratio and enhances the measuring accuracy for the resonant wavelengths. Fig. 5.4 shows seven concatenated 
microscopic snapshots of the shortest microfiber that the author had achieved. The built-in microscope of the microfiber fabrication machine is fixed and used to continuously take the snapshots with a frame width of $0.8 \mathrm{~mm}$ as the holding stages of the microfiber fabrication machine are moved from left to the right simultaneously. The microfiber waist length is estimated to be $2.4 \mathrm{~mm} \pm 0.5 \mathrm{~mm}$. The left and right transitions are estimated to be $2.1 \mathrm{~mm}$ $\pm 0.5 \mathrm{~mm}$ and $1 \mathrm{~mm} \pm 0.5 \mathrm{~mm}$, respectively, which are the most abrupt lengths we were able to achieve. The asymmetrical property is quite common in many microfiber fabrication systems. In our system, since the fiber holding stages are moving with different velocities in the same direction (left stage is faster than the right stage) and the filament is in a fixed position during the fabrication process, it is intuitive that the left transition will be more gradual than the right transition although the lengths of both transitions were set as equal before the fabrication. 

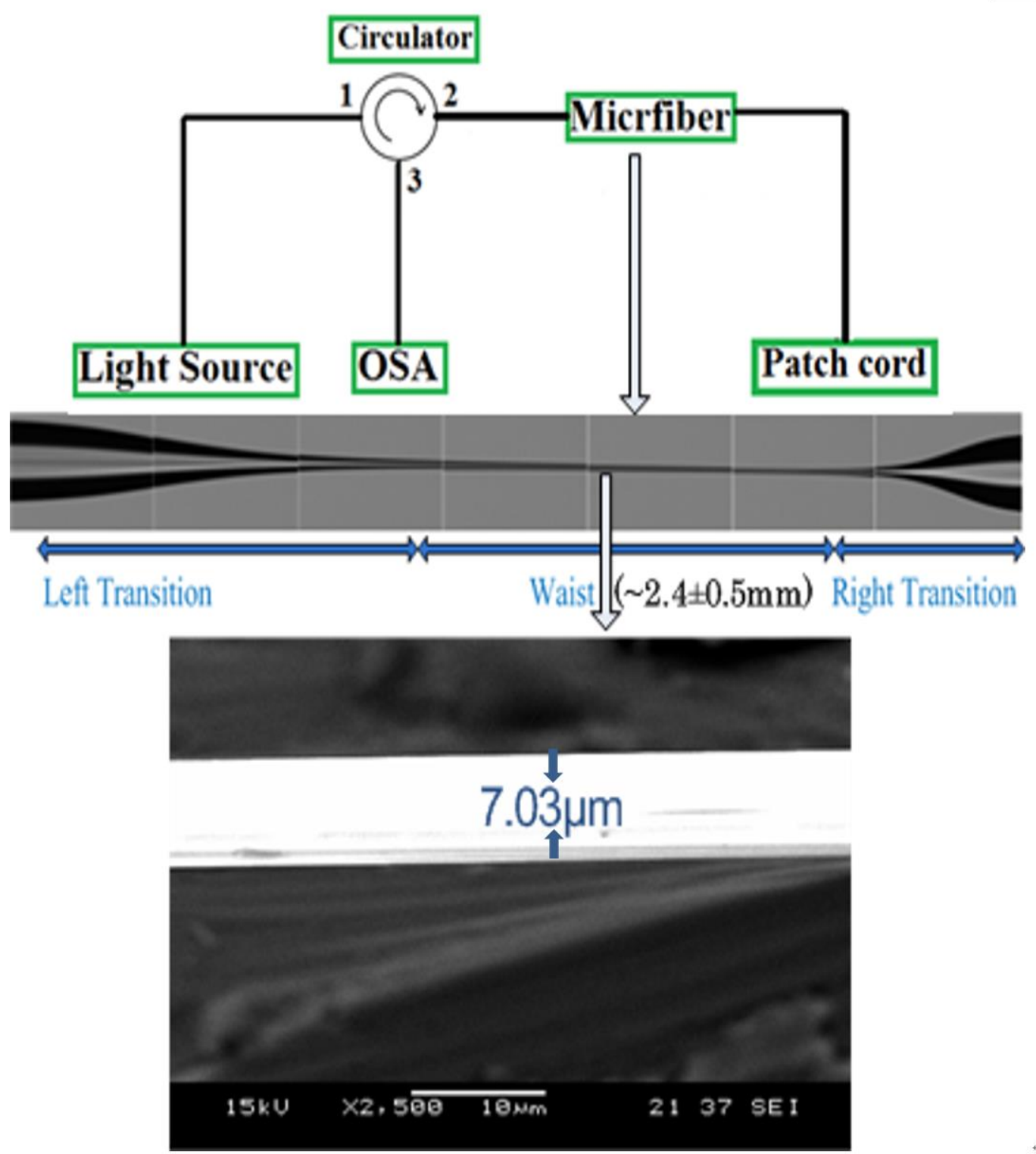

Figure 5.4 Experimental set-up and microscopic snapshots of the ultra-short non-adiabatic microfiber and SEM image of the microfiber waist.

Recall from Eqn. (4.5) that for a fixed microfiber diameter, the FSR would increase as the waist length decreases. This can be verified by the experimental results as illustrated in Fig. 5.5. The four microfibers were fabricated with diameter of $7 \mu \mathrm{m}$ with little deviation in 
their diameters which is insufficient to induce a noticeable difference in their FSRs. The FSR is $37 \mathrm{~nm}$ for a waist length of approximately $4.9 \mathrm{~mm}$. It increased to $80 \mathrm{~nm}$ as the waist length of the microfiber decreased to around $2.4 \mathrm{~mm}$.

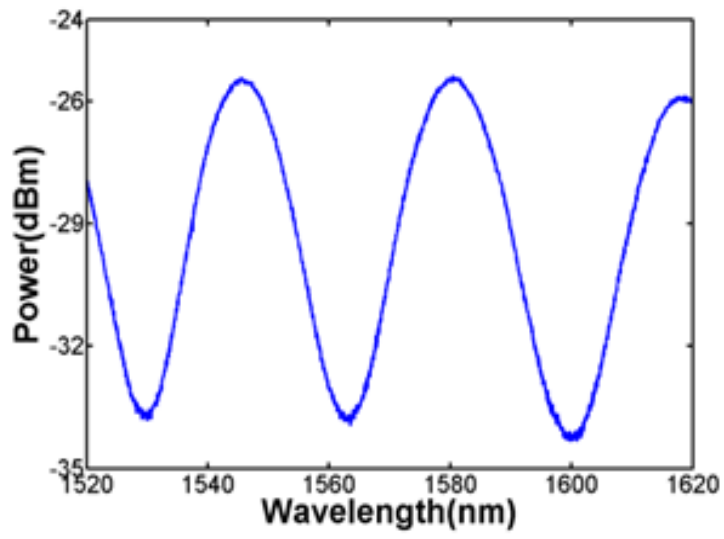

(a)

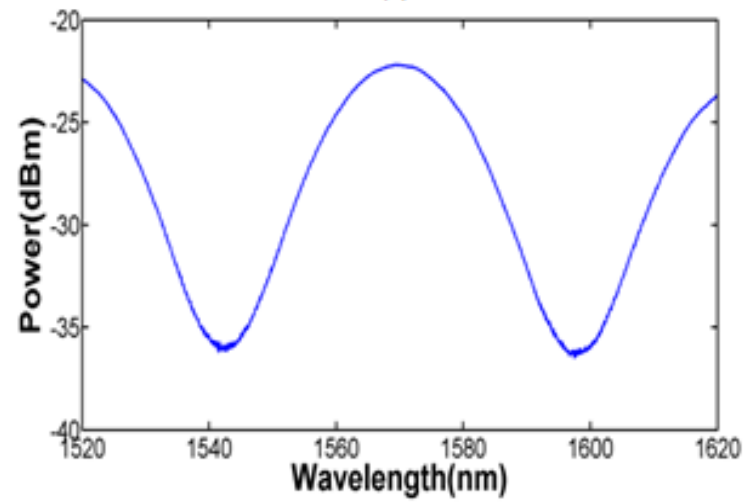

(c)

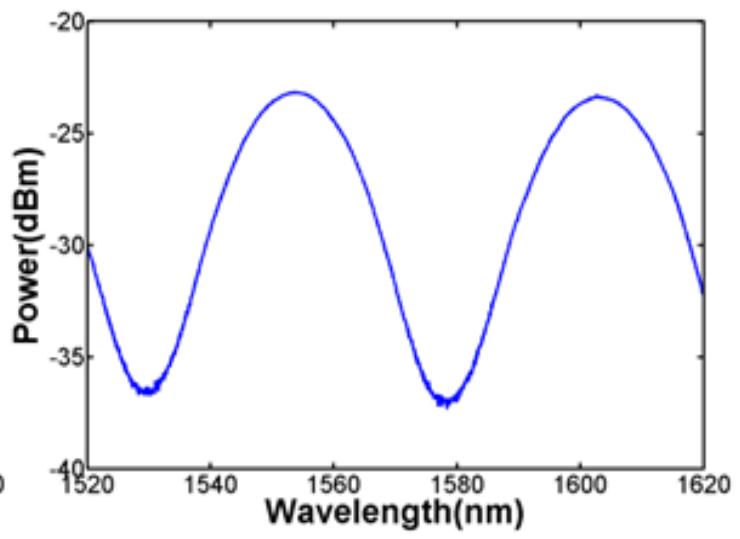

(b)

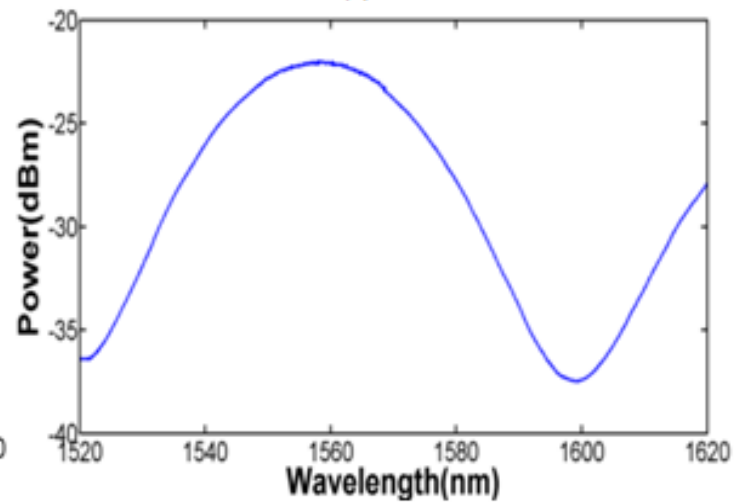

(d) 


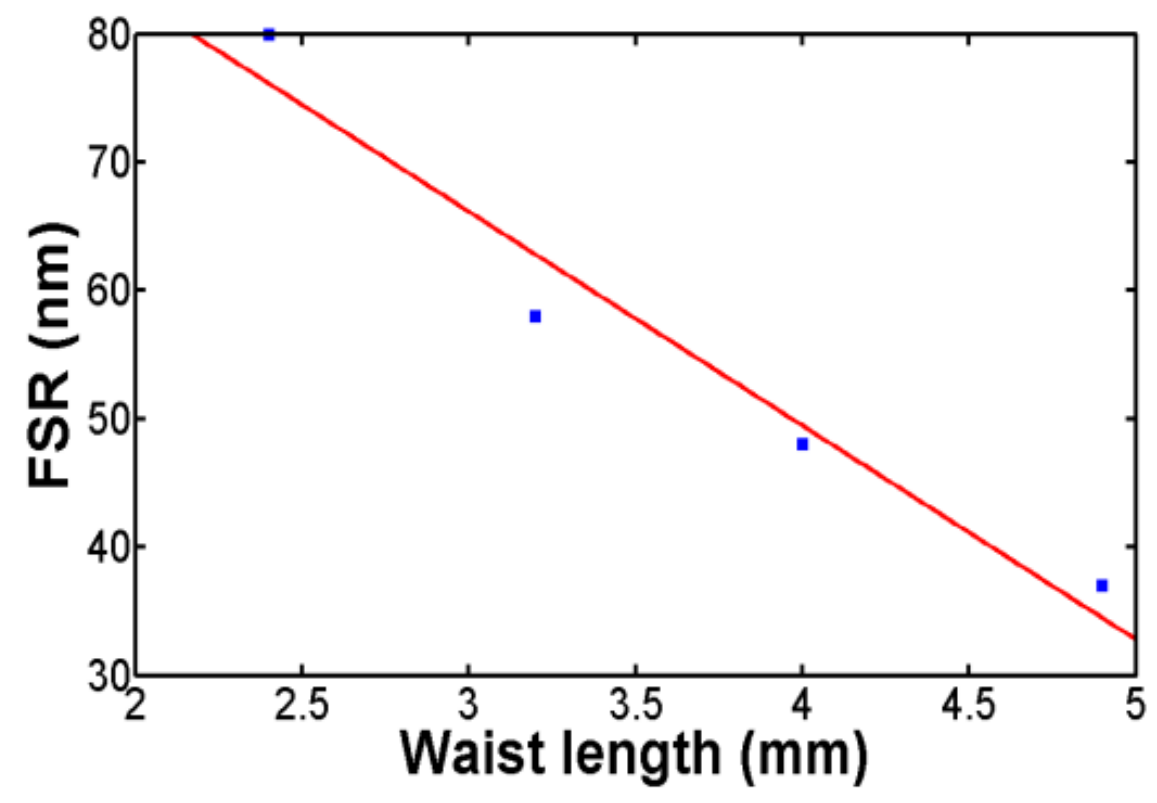

(e)

Figure 5.5 Free spectral range (FSR) of microfibers with diameters of approximately $7 \mu \mathrm{m}$ against (a) Waist length $=4.9 \mathrm{~mm}$ with $\mathrm{FSR}=37 \mathrm{~nm}$, (b) waist length=4 $\mathrm{mm}$ with $\mathrm{FSR}=48$ $\mathrm{nm}$, (c) waist length=3.2 $\mathrm{mm}$ with $\mathrm{FSR}=58 \mathrm{~nm}$, (d) waist length=2.4 $\mathrm{mm}$ with FSR=80 nm, (e) plot of FSR and waist length.

Since the fiber volume is conserved during the fabrication process, there must be a lower bound for the microfiber diameter. When the microfiber length is set to be relatively long, the minimum achievable waist diameter is a relatively small value and vice versa. Fig. 5.6 shows the wavelength response to the RI variations of glycerol solution. For these two microfibers, the filament power and pulling speed were set at $47 \mathrm{~W}$ and $1 \mathrm{~mm} / \mathrm{s}$ (which is the optimal combination as discussed in Table 1). The microfiber in Fig. 5.6 (a) has a waist length of around $4.8 \mathrm{~mm}$. The minimum diameter that we achieved for this waist length was 
$4.85 \mu \mathrm{m}$ and the maximum sensitivity achieved was $52,500 \mathrm{~nm} / \mathrm{RIU}$. For the microfiber in Fig. 5.6 (b) whose waist length is only $2.4 \mathrm{~mm}$, the smallest diameter we have achieved is about $7.03 \mu \mathrm{m}$. The maximum sensitivity achieved was $25,667 \mathrm{~nm} / \mathrm{RIU}$, which is still promising as compared to most of the other optical fiber refractive index sensors that have been reported. More importantly, the FSR of 80nm is larger than most of the interferometric sensors such as the microfiber-based MZI sensor (FSR 22nm) and the microfiber-probebased interferometric sensor (FSR 10nm) [102].

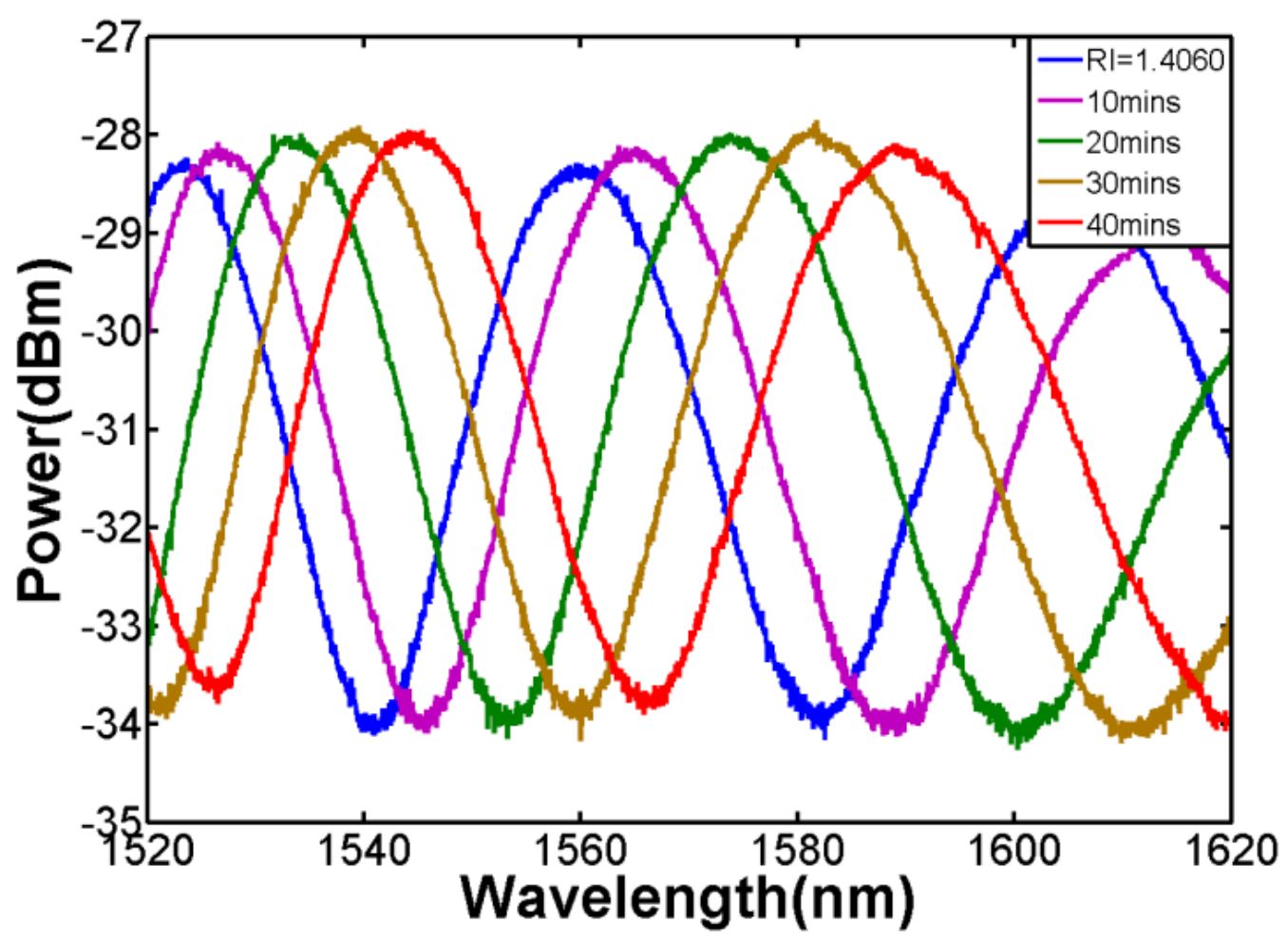

(a) 


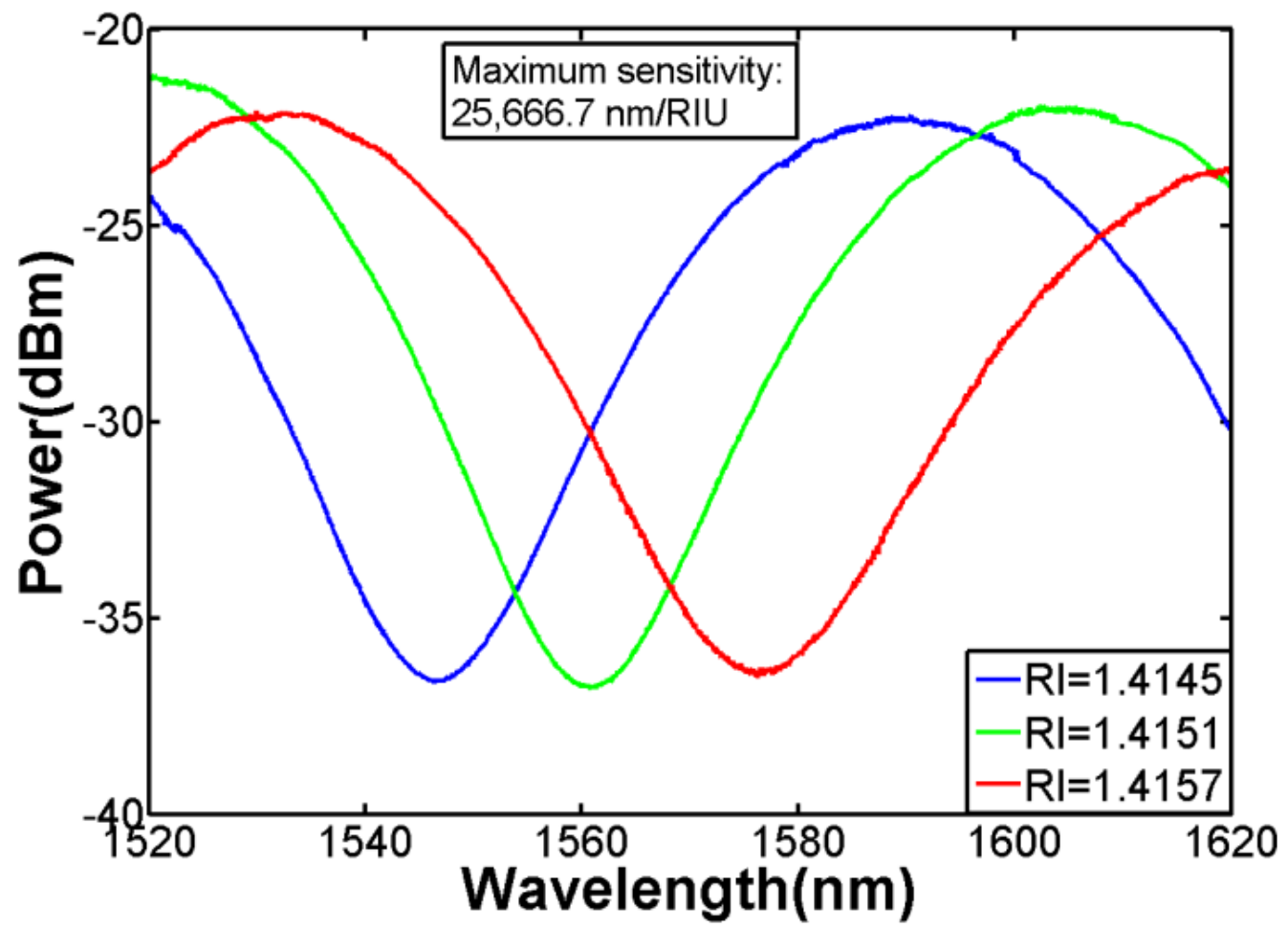

(b)

Figure 5.6. Wavelength responses (a) for natural evaporation of the glycerol solution droplets (The RI of the droplets increase 0.0002 for every 10 minutes) of microfiber with diameter $4.85 \mu \mathrm{m}$ and waist length $4.8 \mathrm{~mm}$. (b) for the microfiber in Fig. 5.4 (d) for different RI of glycerol solution. (Diameter $7.03 \mu \mathrm{m}$, waist length $2.4 \mathrm{~mm}$ )

In summary, the impact of the microfiber fabrication parameters on the microfiber profiles has been studied. It was found that if the filament power were to be set slightly below the normalized power and the pulling speed was set to be faster, it would then be possible to enlarge the FSR without compromising too much of the sensor's performance in terms of its sensitivity. Such a sensor with an ultra-short waist length and a high sensitivity would 
then become more advantageous for sensing applications with small variations in refractive index compared with other interferometric sensors. 


\section{Chapter 6 Detection of heavy metal ions using non-adiabatic microfiber sensor}

\subsection{Overview of techniques for detecting heavy metal ions}

Heavy metal pollution in natural water environment is always a concern in both human and the ecosystem. The current systems available in the market for detecting heavy metal ions all have common drawbacks such as bulky size, expensive set-up and costly maintenance. This work presents a non-adiabatically tapered optical microfiber sensor that is functionalized with chelating agent to detect the existence of specific heavy metal ions of low concentration. Many heavy metals are lethal not only at high concentrations but can be deleterious even at very low concentrations, because they may be accumulated in human organs, causing long term negative health effects [103]. High levels of heavy metal concentration have been found in drinking water in some Asian countries like China, Bangladesh, Thailand, Nepal and India [104]. For instance, high arsenic compound $(107 \mu \mathrm{g} / \mathrm{L})$ found in drinking water consumed by India's population is approximately 11 times higher than maximum permissible limit set by World Health Organization (WHO) [105]. In addition to the need of having a good heavy metal water filtration system, continuous monitoring in the level of heavy metals contamination in natural water environment is also important to ensure safe drinking water for human consumption.

Conventional ways of quantitatively detecting levels of heavy metal contamination include atomic absorption/emission spectroscopy, inductively coupled plasma mass spectrometry and cold vapor atomic fluorescence spectrometry [106]. Even though these methods give good measurement sensitivities, they involve complicated chemical processes 
to extract metal ions from sampled water collected from reservoir of interest. In addition, sophisticated instruments used in these techniques do not encourage on-site detection to be carried out and these instruments are usually very expensive.

As a result, there is a demand of a rapid on-site heavy metal detection system that is low in cost and does not involved complicated operation procedures. One plausible solution for this matter is to use optical sensor owing to the fascinating characteristics of optical fiber sensors including its compact size and light weight, suitability for use in harsh environment, and remote sensing capability[107]. Various kinds of optical fiber structures, each with unique design and optical properties have been explored to fit the needs of a particular sensing system.

The key figure of all chemical sensors is the known chemical compound used to identify and quantify the target analyte. These compounds were wisely chosen for their specificity to the target analyte. Moreover, they should also be able to convert successful detection of analyte into a measurable physical change for analytical purposes. These chemical compounds which are usually organic (i.e. compounds whose molecules contain carbon) in nature are immobilized onto the sensing surface, and the means used to attach them to the surface plays a critical role in sensor development and optimization. Many developed fiber optic chemical sensors found in the literature often involved chemical modification of the optical fiber surface $[108,109]$. This is because common optical fiber is made of inorganic material and there is a need to functionalize the surface prior to attachment of organic substrate/compound on it. Once the fiber surface is ready to bond with organic substrate/compound, a crosslinker, which acts as a bridge, is required to create a strong bond 
between them. The substrate/compound layer developed on the fiber surface will react with target analyte, causing variation in the intensity of the light travelling down the fiber. Quantitative analysis can be done since the intensity change is related to the analyte concentration.

In this work, chelating agents for detecting heavy metal ions were coated on microfiber surface which has the diameter of less than $4 \mu \mathrm{m}$. Chelating agents are organic compounds, often used in metal intoxication medical treatment due to their ability to form a stable metal chelates (i.e. compound composed of metal ion and chelating agent) that are easily excreted from the target site. Although several researchers have demonstrated surface-modified tapered microfiber as heavy metal sensor and low detection limits were reported [109, 110], chemical functionalization on such small diameter silica fiber was still a new area to explore, to the best of the author's knowledge. Besides the coating process itself being a challenge, the cross sensitivity of such microfiber is another issue that needs to be addressed properly. The strong evanescent field exposed to the surrounding makes it sensitive not only to refractive index but also to strain and temperature $[39,100,111]$. The current protocols of coating chelating agents in each coating stage was successfully modified and also a system was designed to eliminate the cross sensitivity effect, in such a way that the results obtained are promising and reliable. 


\subsection{Experiments}

\subsubsection{Fabrication of microfiber}

For refractive index sensor, the microfiber needs to be tapered to as small a diameter as feasible in order to achieve high sensitivity. However, for most of these interferometer-based sensors, there is one common drawback in terms of the measuring range of the refractive index. As the wavelength responses of these modal interferometers are periodic, once the wavelength shift due to the change of external medium exceeds the free spectral range (FSR), it is impossible to identify how much the resonant wavelength has shifted. In Chapter 5, the author has discussed that the most dominant parameters to form a desired fiber taper are the heating power and the pulling speed. By proper control of these parameters, the issue of the trade-off between the sensitivity (determined by microfiber radius) and the measuring range (determined by FSR) has been resolved by tapering an ultra-short waist microfiber [112].

In the microfiber fabrication process, the tension in the microfiber was always monitored so it is crucial for calibrating the normalized power of the graphite filament as well as its respective power degradation rate. The power is close to the normalized value when the fiber tension detected by the monitor during the fabrication process is maintained at a certain positive level with slight periodic fluctuations. In this work, the normalized power of the filament was calibrated as $50 \mathrm{~W}$ and the power degradation rate is $-15 \%$ during the waist drawing period. The 
fabricated microfiber has the diameter of only $3.9 \mu \mathrm{m}$ and the FSR is $20 \mathrm{~nm}$ which is large enough for detection of slight change in fiber surface [112].

\subsubsection{Microfiber surface modification}

Surface treatment of fabricated microfiber was accomplished by soaking the microfiber into engineered chemical solvents. The treatment process begins by washing the microfiber in acetone solution for ten minutes in order to remove dust particles, contaminants or other impurities on the fiber surface. Prior to silanization, the microfiber was cleaned with $1 \mathrm{M}$ sulfuric acid $(\mathrm{H} 2 \mathrm{SO} 4)$ for 30 minutes at $90^{\circ} \mathrm{C}$, followed by Piranha solution (mixture of sulfuric acid and hydrogen peroxide in a volume ratio of 3:1) for 10 minutes inside a fume hood and $1 \mathrm{M}$ sodium hydroxide $(\mathrm{NaOH})$ for 10 minutes at $120^{\circ} \mathrm{C}$. Between each of this cleaning steps, the microfiber was rinsed thoroughly a few times with deionized water. This step is important to form high density of hydroxyl functionalities necessary for silane modification. Next, two percent of silane coupling agent, 3-Aminopropyltriethoxysilane (APTES) was mixed into deionized water at $\mathrm{pH}$ 4.5-5.5 (adjusted by acetic acid). The microfiber was dipped into the solution for two hours at $75^{\circ} \mathrm{C}$ under low humidity environment. Silanized microfiber was then dried overnight in an oven at $60^{\circ} \mathrm{C}$. Following this, each $\mathrm{ml}$ of carboxyl groups containing chelating agent solution such as Ethylenediaminetetraacetic acid (EDTA) were activated using $0.4 \mathrm{mg}$ of 1-ethyl-3(3-dimethylaminopropyl) carbodiimide hydrochloride (EDC) and stabilized by $1.1 \mathrm{mg}$ of N-hydroxysuccinimide (NHS) and left undisturbed for 15 minutes at room temperature. The activated chelating agent was then added to the silanized 
microfiber and left to stand for 2 hours to allow covalent bonds to be formed. At the end of the bonding reaction, hydroxylamine, in a final concentration of $10 \mathrm{mM}$, was then added to stop the reaction. An illustration of this surface functionalization protocol is shown in Fig. 6.1.

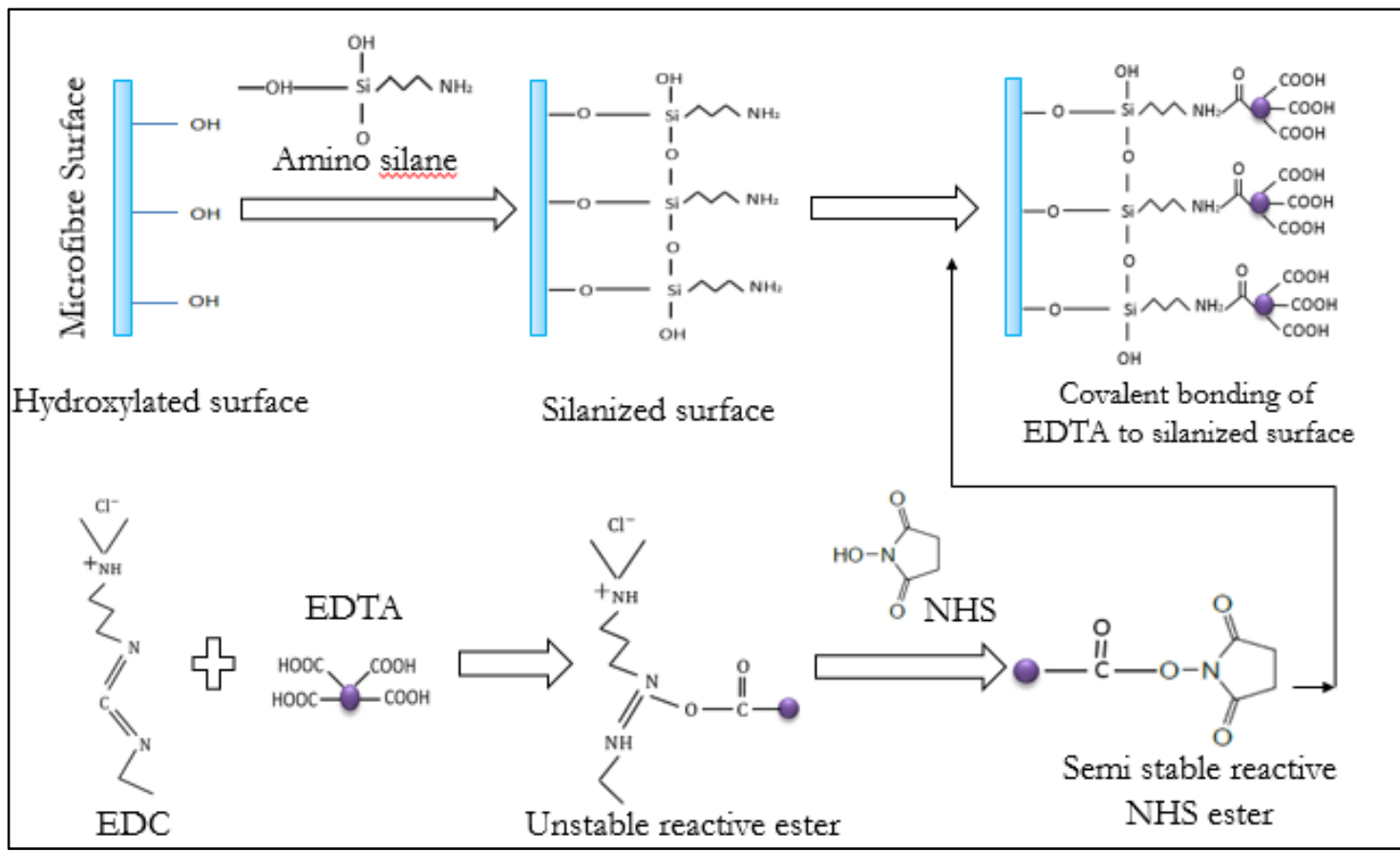

Figure 6.1 Surface functionalization of microfiber by silanization and the reaction scheme of EDC/NHS coupling for bonding of chelating agent onto the surface

\subsection{Scanning Electron Microscope (SEM) imaging}

Fig. 6.2(a)-(b) shows the SEM images of the surface of fiber samples at different stages of the functionalizing process. Under large magnification, a uniform layer of APTES can be seen on the silanized sample, which is critical for the attachment of chelating agent. In addition, Fig. 6.2(c) shows a fiber sample that has been immersed 
into lead (II) ion solution. From these figures, apparent increase in surface roughness was seen as the fiber underwent more stages of chemical functionalization.

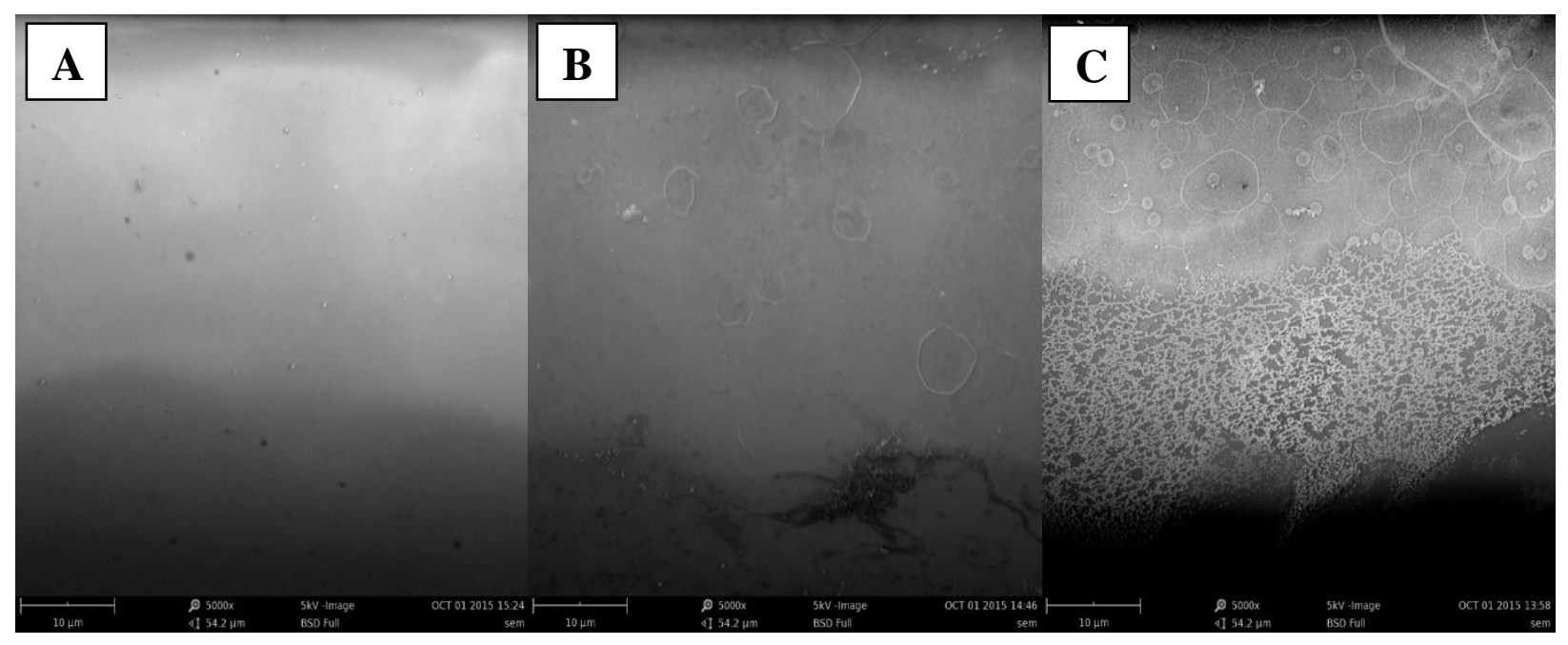

Figure 6.2 (a)-(c): SEM image with magnification $\times 5000$ of (a) silanized fiber using APTES (b) functionalized fiber with EDTA attached on the surface and (c) functionalized fiber with lead (II) ion trapped on the surface by EDTA

\subsection{Energy dispersive X-Ray Spectroscopy (EDX) Analysis}

In Table 2, all element compositions found on the non-functionalized and functionalized microfiber were listed. Silanized microfiber showed a dramatic 
increase in carbon element compared to non-functionalized microfiber. Silanization of microfiber with APTES was carried out to form silanol and amino sites, as seen in Fig. 6.3. Thus, results obtained demonstrated that APTES had reacted with the hydroxyl group on the microfiber surface. In addition, the ratio of silicon and oxygen atom is approximately $1: 2.5$. This confirmed that APTES was bonded to two or three active hydroxyl sites.

\begin{tabular}{|c|c|c|c|c|}
\hline $\begin{array}{c}\text { Element } \\
\begin{array}{c}\text { Weight } \\
(\%)\end{array}\end{array}$ & $\begin{array}{c}\text { Non- } \\
\text { functionalized } \\
\text { Microfiber } \\
\text { (Fig. 6.4(a) })\end{array}$ & $\begin{array}{c}\text { Non-functionalized } \\
\text { Microfiber Tested } \\
\text { with } \mathrm{Pb}^{2+} \text { solution }\end{array}$ & $\begin{array}{c}\text { Silanized } \\
\text { Microfiber } \\
\text { (Fig. 6.4(b)) }\end{array}$ & $\begin{array}{c}\text { Functionalized } \\
\text { Microfiber } \\
\text { Tested with } \\
\mathrm{Pb}^{2+} \text { solution } \\
\text { (Fig. 6.4(c)) }\end{array}$ \\
\hline $\mathrm{O}$ & $69.55 \pm 1.25$ & $68.90 \pm 1.23$ & $53.71 \pm 1.45$ & $64.47 \pm 1.22$ \\
\hline $\mathrm{Si}$ & $24.63 \pm 0.59$ & $21.52 \pm 0.52$ & $21.66 \pm 0.65$ & $24.87 \pm 0.55$ \\
\hline $\mathrm{C}$ & $5.82 \pm 1.55$ & $9.58 \pm 1.48$ & $26.64 \pm 1.76$ & $9.39 \pm 1.54$ \\
\hline $\mathrm{Pb}$ & $\mathrm{nd}$ & nd & nd & $0.78 \pm 0.28$ \\
\hline
\end{tabular}

\section{*nd-not detected}

Table 2: Microscopic composition of microfiber at the scanning area 


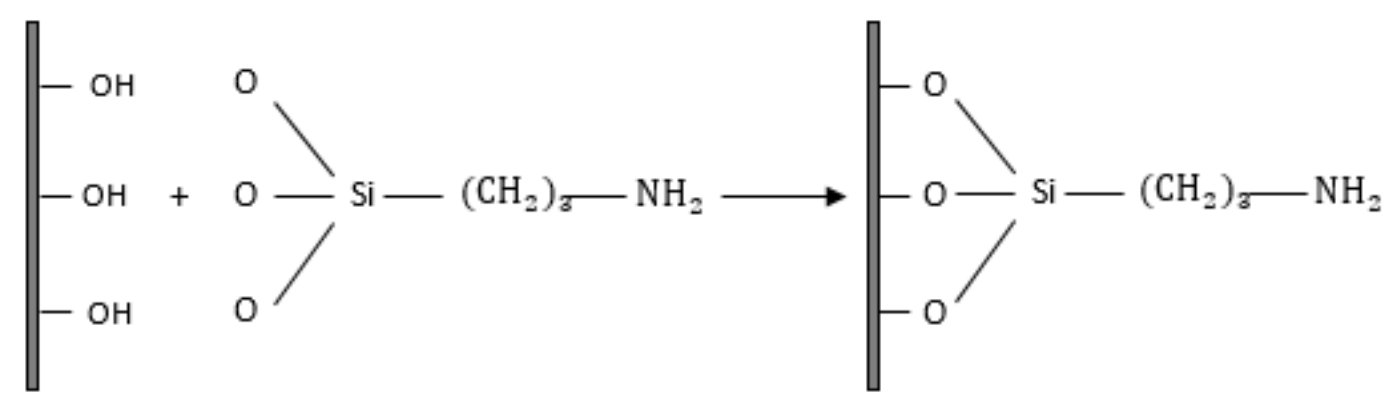

Figure 6.3 The possible silanization process of microfiber surface

In order to verify the capability of functionalized microfiber to chelate heavy metal ion, non-functionalized and functionalized microfiber was dipped separately into lead (II) solution and rinsed thoroughly with deionized water, followed by EDX analysis. Results shown in Table 2 and Fig. 6.4 indicated there was no trace of lead element found on the non-functionalized microfiber. Conversely, about $0.78 \pm 0.28 \%$ was detected on the surface of EDTA functionalized microfiber. For this reason, the ability of functionalized microfiber sensor for trapping heavy metal ion onto the sensing region was proven. 

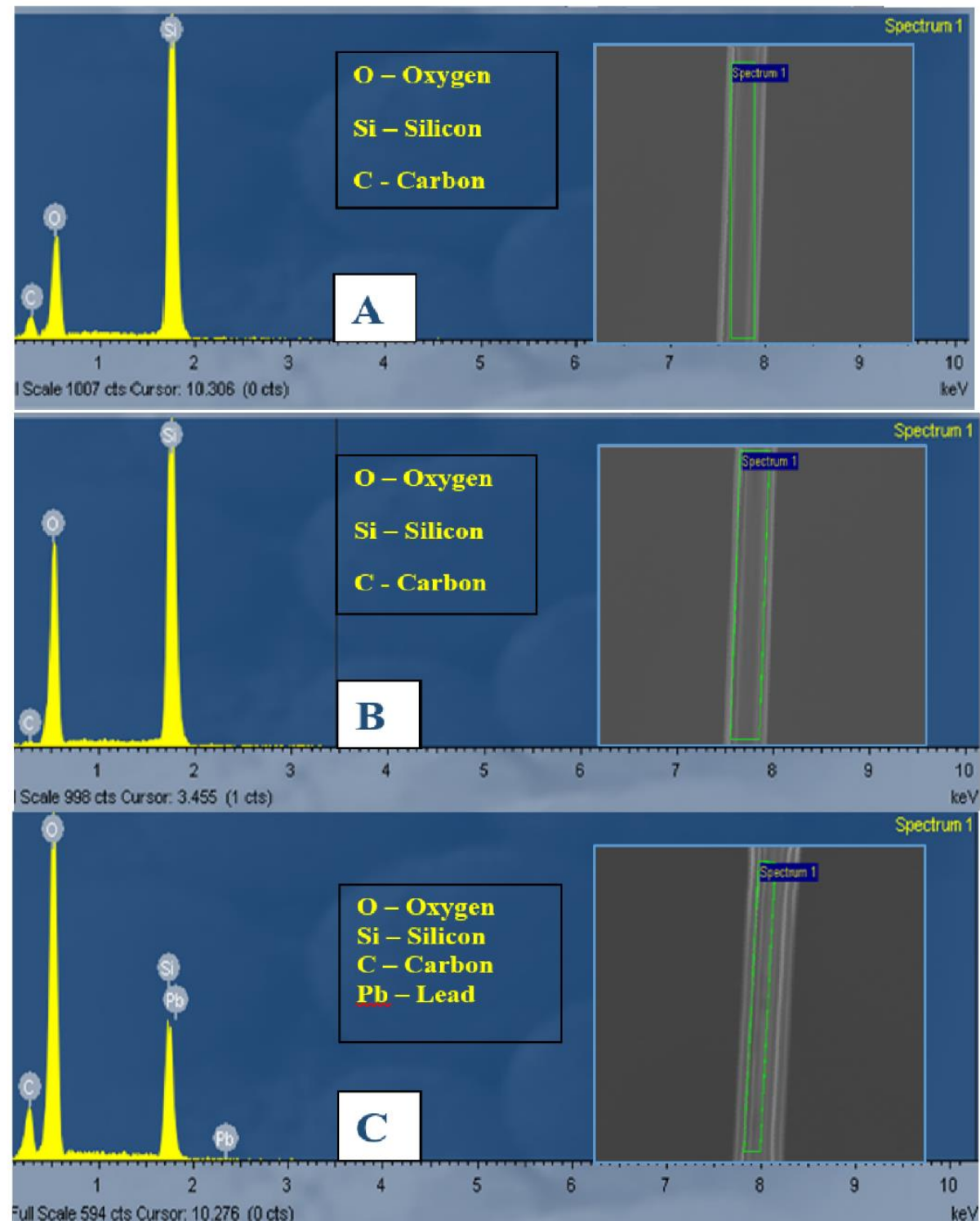

Figure 6.4 (a)-(c) The spectra of microfiber sensor obtained from SEM-EDX analysis. Green color frame in the inset diagram represents the scanning area 


\section{Chapter 7 : Characterization of surface functionalized microfiber sensor}

\subsection{Characterization}

Fig. 7.1 shows the schematic diagram of the experimental setup of the microfiber sensor. Light from laser source is coupled into port 1 of the circulator to port 2 towards the microfiber. The other end of fiber is terminated with a fiber patch cord. The effect of terminating with a patch cord in this work is similar to coating a layer of gold onto the end facet of the fiber, with the only difference being the lower reflectivity but more cost-effective. The reflected light from the microfiber is coupled via port 3 of the circulator to an optical spectrum analyzer (OSA). As mentioned in previous section, microfiber sensor is usually sensitive to refractive index, strain and temperature concurrently. Fig. 7.2 demonstrates the strain and temperature sensitivities of two microfibers. The maximum sensitivity for strain is approximately $-15 \mathrm{~nm} / \mathrm{mE}$ and maximum sensitivity for temperature reaches -380

$\mathrm{pm} /{ }^{\circ} \mathrm{C}$. As the refractive index is the only target that we are investigating in this section the cross-sensitivities must be minimized. The design for addressing such issue is shown in Fig. 7.1. In the sensing region, the surface-functionalized microfiber was sealed in a cartridge fabricated via a 3D printer using Acrylonitrile Butadiene Styrene (ABS), which is a type of plastic material. The slot in the center is a fluidic channel that is designed by padding Polydimethylsiloxane (PDMS) sheets on top and bottom of the cartridge. The water sample was injected by a syringe through the top PDMS sheet and discharged through a pipe embedded in the bottom PDMS sheet. The outflow in the pipe was controlled by a clip and directed into a beaker underneath the cartridge. One pair of the magnetic clips was deployed inside 
the edges of the cartridge to provide primary strain control. The fiber was then fixed by another pair of magnetic clamps mounted on the translation stages that were screwed on magnetic holders. By moving the translation stages, a secondary strain control was provided. Such design resolved the issues of cross sensitivities caused by temperature and strain. The closed environment of the cartridge minimizes the influence of ambient temperature fluctuations on the microfiber while the magnetic buttons and the magnetic clamps minimizes the stain effect. Nevertheless, careful characterization must be done each time before measurement to minimize cross sensitivities, especially the strain effect. From experience, the author has found that when the microfiber is not properly tensioned, it wobbles when the water sample was pumped into the channel resulting in an unstable spectrum in the OSA. Hence, a certain amount of tension needs to be applied to the microfiber and it should be precisely controlled to avoid breaking the microfiber. The tension was applied by moving the translation stage away from the center and monitoring the wavelength response in the OSA. The spectrum will only start to change once the microfiber is in tension as shown in Fig. 7.2(a). The negative tensions are counted as zero. In all experiments, the strain was set at $3 \mathrm{mE}$. 


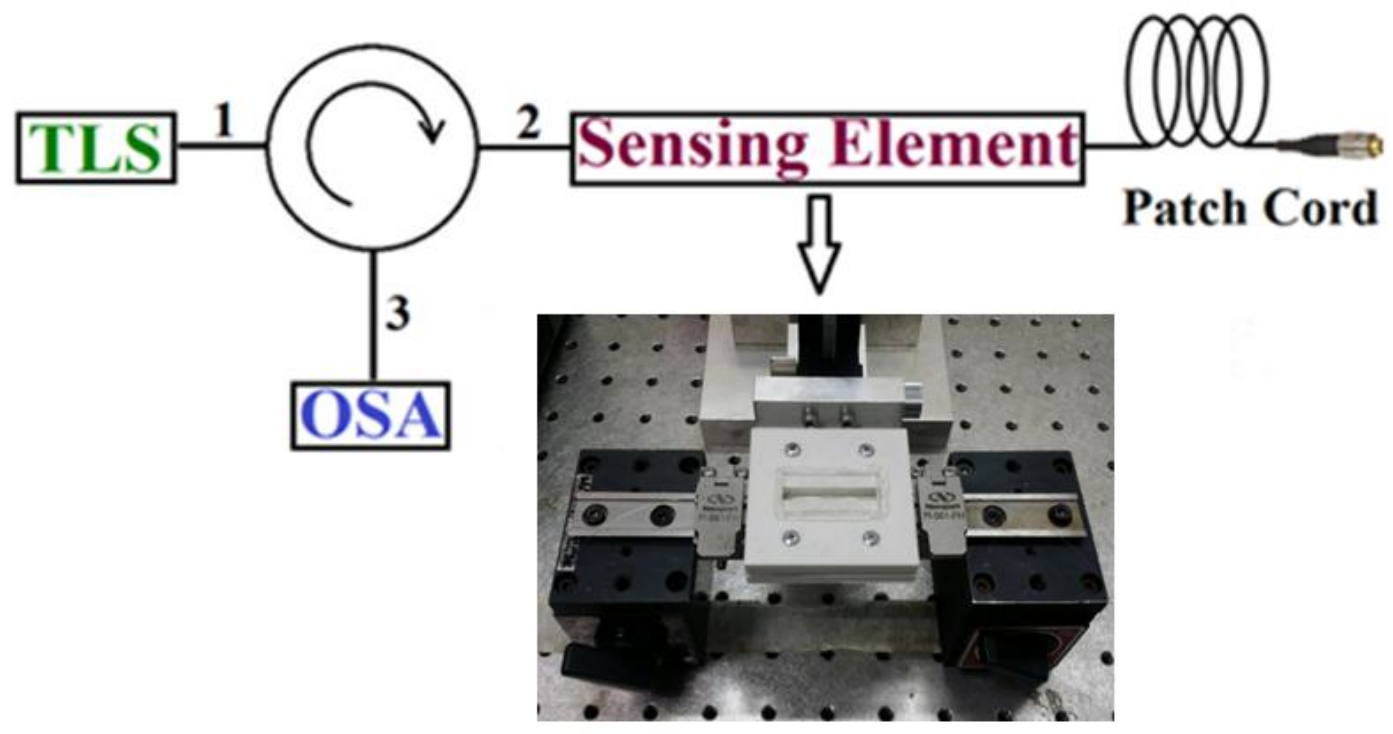

Figure 7.1 Microfiber sensor system set-up

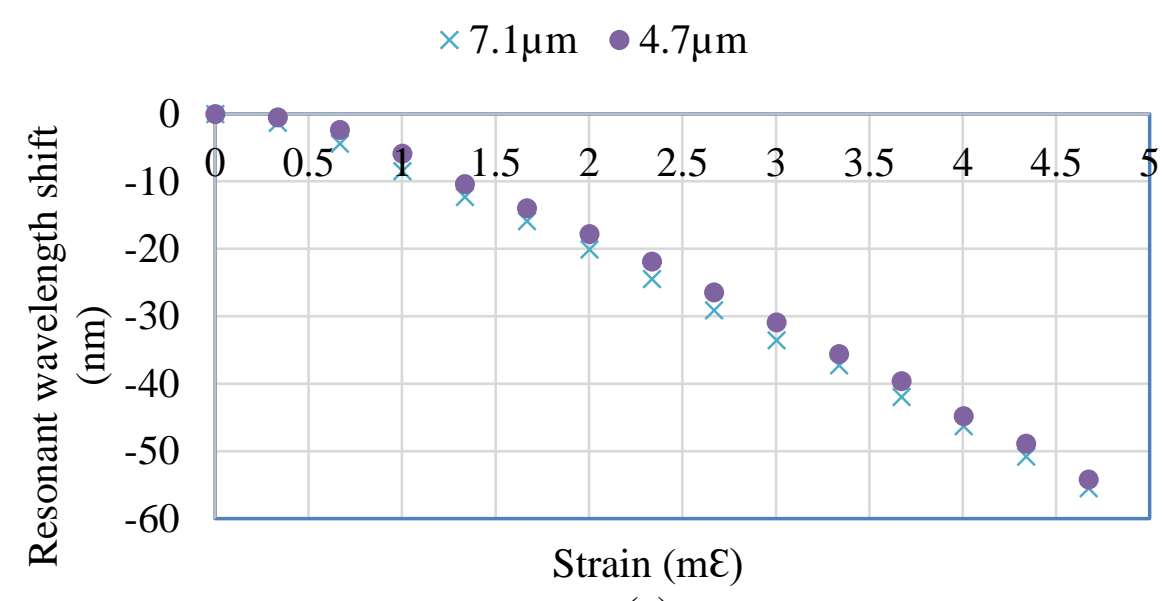

(a) 


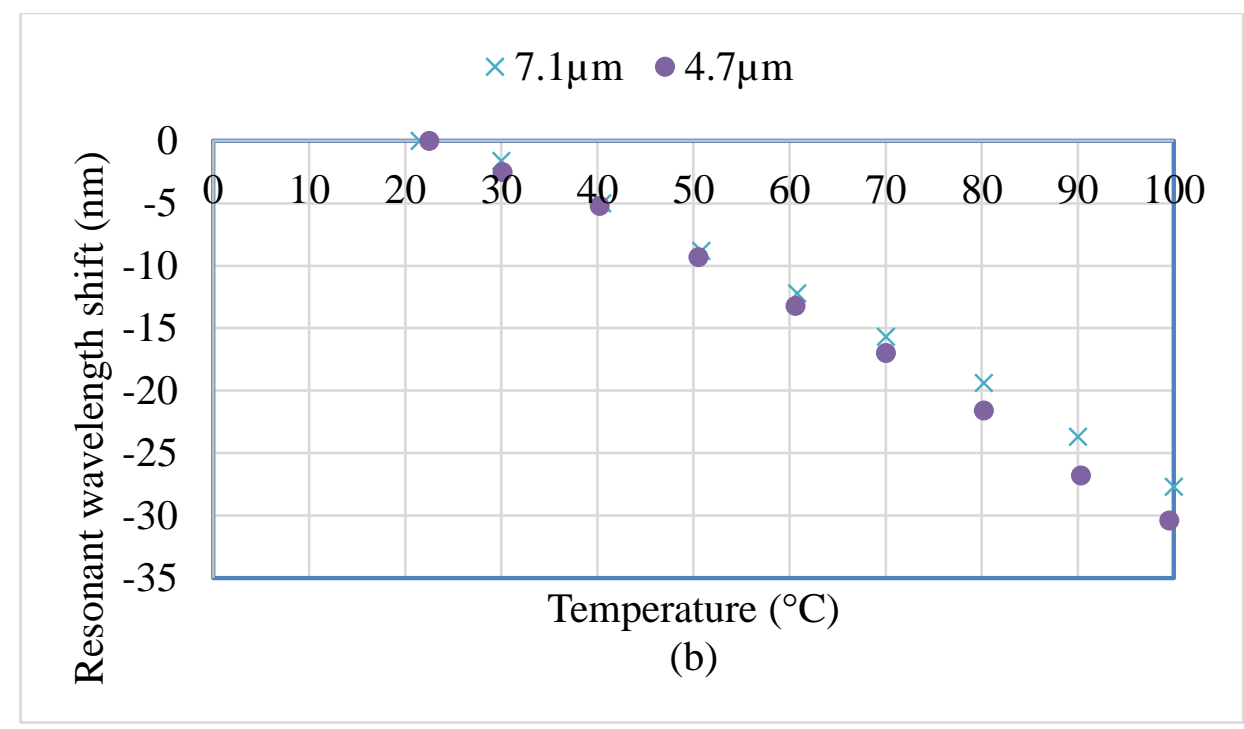

Figure 7.2 Characterization of (a) Strain sensitivity (b) Temperature sensitivity

\subsection{Wavelength responses for non-functionalized and functionalized microfiber} sensor

Having the strain and temperature issues addressed, the water samples with heavy metal ions were characterized. A reference spectrum was recorded by immersing the microfiber in de-ionized water before the metal ion solution was tested. After doing so, we left the surface functionalized microfiber sensor to dry in air for 5 minutes before pumping the metal ion solution into the reservoir. The solution was discharged 30 minutes later through a tube underneath the cartridge and the microfiber was flushed by DI water for a few times. Subsequently, the microfiber was dried for 5 minutes and immersed to the DI water again. The spectrum was recorded once it is stabilized. The resonant wavelength shift was observed and compared to the reference spectrum. The reason of recording the spectrum of the microfiber in DI water again after the reaction between the chelating agent and the heavy metal ions is to avoid uncertain factors in the water sample such as the 
refractive index difference with DI water. As demonstrated in previous study [39], non-functionalized microfiber is also capable of detecting the surface refractive index change but lacks the ability to determine the concentration of specific molecules in the sample. Hence, the performance of the microfiber before it was functionalized was also characterized. Fig. 7.3 shows the sensitivity comparison between the non-functionalized and functionalized microfiber. It can be seen that the microfiber sensor functionalized with chelating agent EDTA has much higher sensitivity than the non-functionalized one. One thing worth noting is that the nonfunctionalized sensor showed no wavelength shift when low concentration metal ion solution is used, while a clear wavelength shift was observed for the functionalized sensor. 


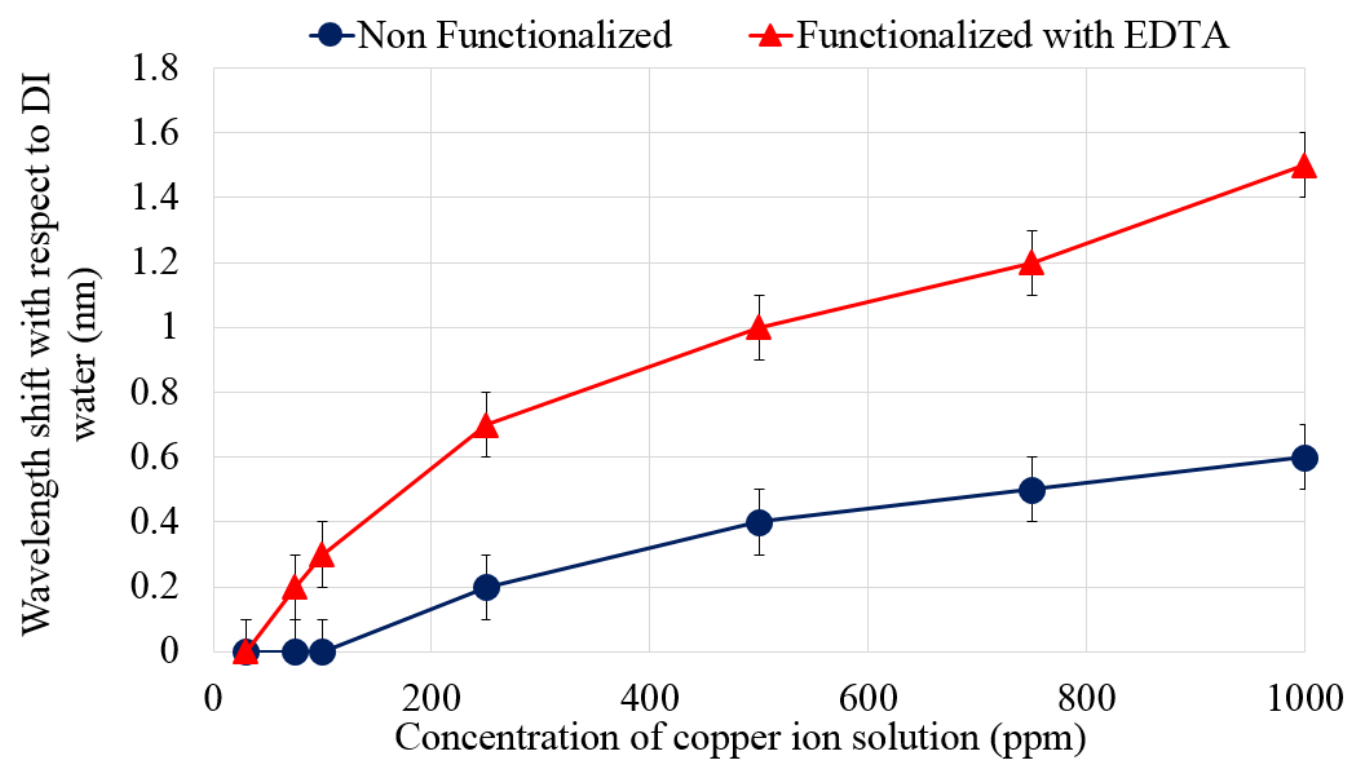

Figure 7.3 Sensitivity comparison between non-functionalized and functionalized microfiber sensors

\subsection{Comparison of coating different chelating agents}

As each type of chelating agent has its binding stability constant to specific metal ions, the author has also investigated microfiber sensors functionalized with different chelating agents and the results are shown in Fig. 7.4. It shows that microfiber sensor with D-Penicillamine (DPA) bonded onto its surface greatly enhances the detection limit of copper (II) ions. Abundance of literatures describes the effectiveness of DPA, whether in the excretion of copper in animals or environmental study [113, 114]. The reason behind this could be that DPA-copper (II) complex is formed through a reversible addition of bidentate ligand by means of DPA's thiol and amine group. At this stage, electrons of $\mathrm{Cu}-\mathrm{S}$ transfer reaction will take place, causing the metal ion to reduce to copper (I). The reduction process causes a weaker bond of 
copper (I) (since total charge becomes less and thus, less ionic contribution to the bond) and appear more accessible for the attachment to other penicillamine molecules. Thus DPA possesses higher chelative capacity in comparison to EDTA and is deemed more effective in extracting copper ion from a compound [113].

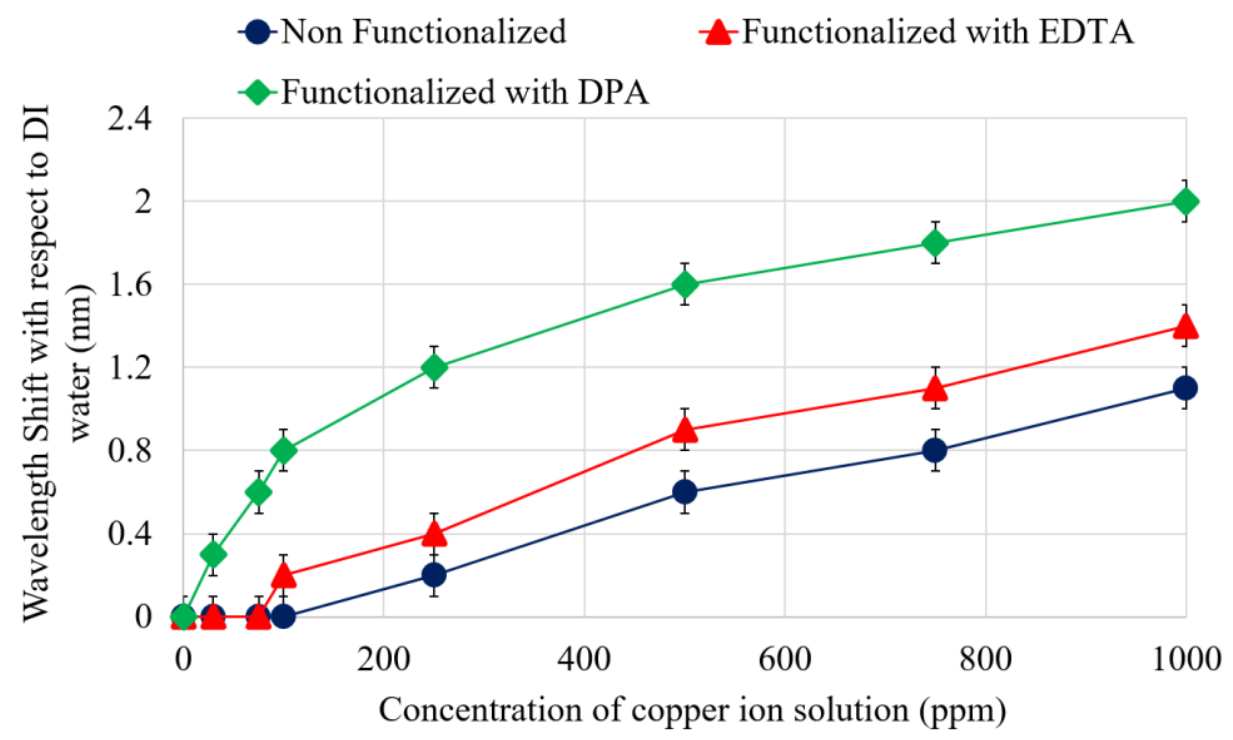

Figure 7.4 Sensitivity comparison between different chelating agents

\subsection{Detection limit}

The author has tested many types of metal ions including Lead (II), Copper (II), Zinc (II) and Cadmium (II) ions with concentrations ranging from $10 \mathrm{ppb}$ to 100ppb using different chelating agents coated on very thin microfibers. Fig. 7.5 shows examples of these four metal ions detection results. In Fig. 7.5 (a), a wavelength shift of around $0.2 \mathrm{~nm}$ was observed for 10 PPB Copper (II) ion solution utilizing a microfiber $(3.9 \mu \mathrm{m}$ in diameter) coated with DPA. According to drinking water quality guidelines prepared by organizations and committees throughout the world, the maximum concentrations of Copper (II) ion allowable in public drinking water 
system are 2 ppm for World Health Organization [101] and $1.3 \mathrm{ppm}$ for United States Environmental Protection Agency [103]. The safety margin for the sensor's detection limit clearly meets these standards.

However, the resolution is not as good as copper ion for rest of the metal ions that we have tested, as shown in Fig. 7.5(b)-(d). The reason may be due to the selectiveness of chelating agent to metal ions as discussed in previous section. The stability constant between chelating agents and metal ions determines how strong the covalent bonding has formed between metal and the ligand. DPA has a high stability constant with copper ion thus the DPA-functionalized microfiber surface could trap more copper ions even when the concentration is low [113]. EDTA has moderate stability constants with many metal ions including zinc, lead and cadmium therefore the minimum concentration detected is not as low as copper which was detected by DPA-functionalized microfiber. Moreover, one thing that needs to be declared that our sensor does not yet have the capability of simultaneously sensing multiple types of metal ions in one water sample as the chelating agent is usually sensitive to many metal ions which causes cross-sensitivities. However, with proper choosing of the metal-selective chelating agents, it could be still possible to multiplex a few microfiber sensors coated with different chelating agents to detect multiple metal ions. 


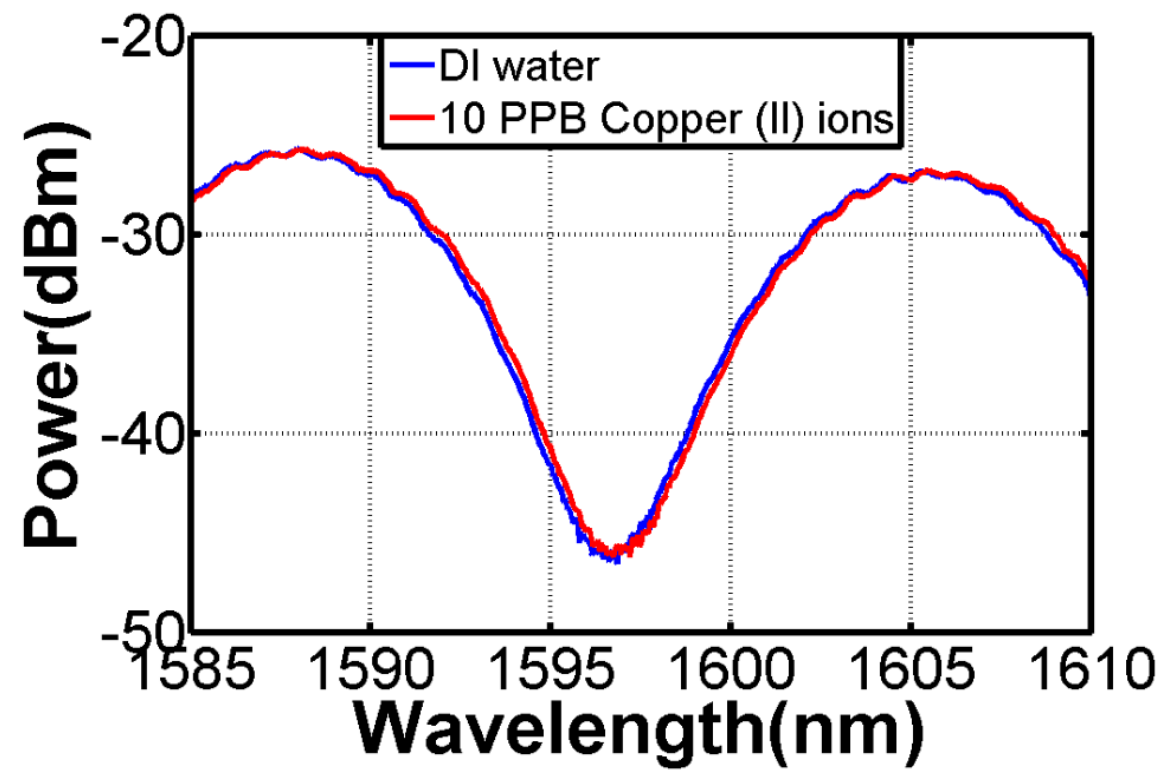

(a)

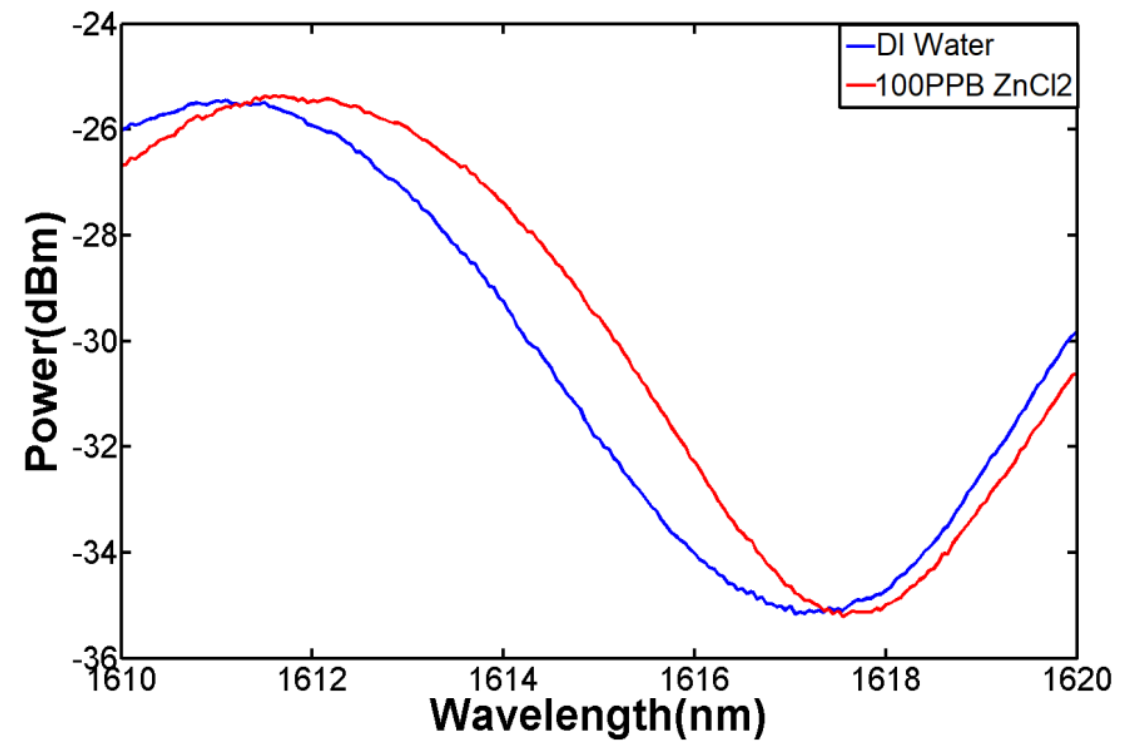

(b) 


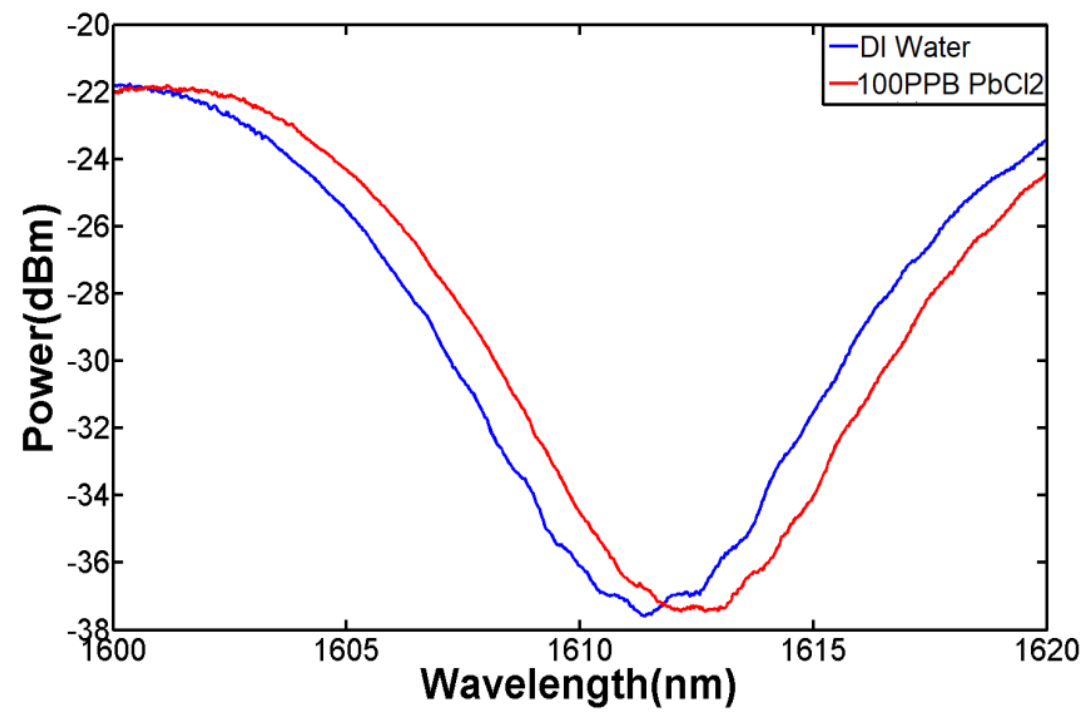

(c)

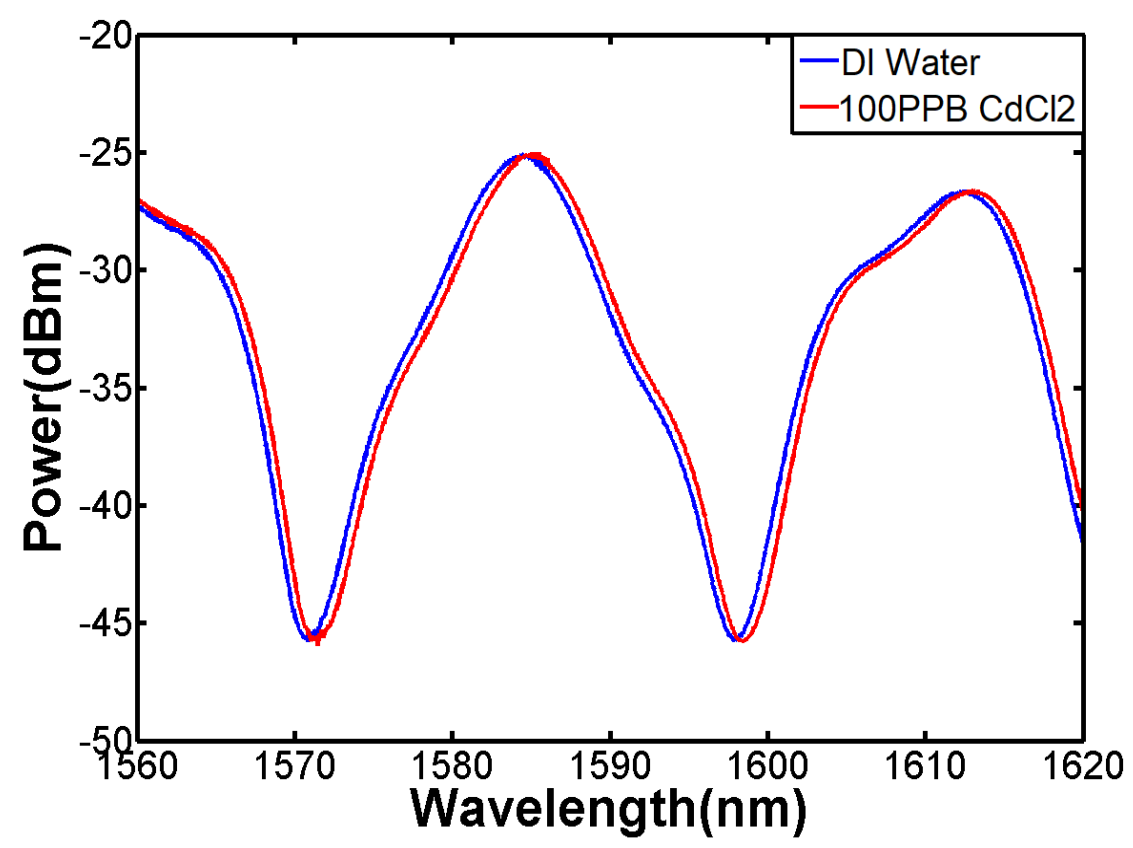

(d)

Figure 7.5 Wavelength spectra for (a) DI water and 10 ppb copper (II) ion solution using microfiber sensor with diameter of $3.9 \mu \mathrm{m}$ coated with DPA (b) DI water and $100 \mathrm{ppb}$ zinc (II) ion solution using microfiber sensor with diameter of $3.9 \mu \mathrm{m}$ coated with DPA (c) DI water and 100 ppb lead (II) ion solution using microfiber 
sensor with diameter of $3.9 \mu \mathrm{m}$ coated with EDTA (d) DI water and 100ppb

cadmium (II) ion solution using microfiber sensor with diameter of $3.9 \mu \mathrm{m}$ coated with EDTA 


\section{Chapter 8 : Conclusion and recommendation for future work}

\subsection{Conclusion}

In summary, the author has studied two types of microfiber sensor, namely the adiabatically and non-adiabatically tapered microfiber sensors. For adiabatic microfiber sensor, MFBGs were successfully fabricated in microfibers which were tapered from a single mode fiber as well as multi-mode fiber. This work aims to explore more applications of FBG. It did show that the FBG can sense not only strain and temperature but also refractive index although the sensitivity is not very high. Long period gratings were also investigated by inscribing it in a photosensitive specialty fiber with a W-shaped refractive index profile for sensing of refractive index and temperature. The spectra for both terms differ slightly from conventional LPGs. The resonant wavelength shifts towards the longer wavelength when external refractive index increases or temperature increases. The sensor achieves a remarkable resolution of $1.67 \times 10^{-5}$ for refractive index sensing and sensitivity of 57 $\mathrm{pm} /{ }^{\circ} \mathrm{C}$ for temperature sensing. Although the fiber is tapered into much smaller diameter, the change in taper diameter is gradual enough to ensure the robustness of the fiber in terms of the mechanical strength.

For non-adiabatic microfiber sensor, an RI sensor was fabricated by tapering a single-mode fiber into a few micrometers with abrupt taper transitions. The microfiber sensor has shown extraordinary sensitivity. The high sensitivity in the RI 
ranging from 1.3337 to 1.37 could be useful in detecting contamination of water, monitoring leakage for oil companies and so on. Moreover, the fabrication process is simple and the sensor is generally compact and robust. Subsequently a method of improving the measuring range of refractive index was also proposed which makes such sensor more applicable for real situations. The impact of the microfiber fabrication parameters on the microfiber profiles has been studied. It was found that if the filament power were to be set slightly below the normalized power and the pulling speed was set to be faster, it would be possible to increase the FSR while keeping the microfiber's radius small. The author has successfully fabricated ultra-short microfiber without compromising too much on the sensitivity. The waist length of our microfiber is $2.4 \mathrm{~mm}$ which is almost equivalent to the limit of the filament width $(2 \mathrm{~mm})$. The diameter of the microfiber is around $7 \mu \mathrm{m}$ and the maximum sensitivity obtained is $25667 \mathrm{~nm} / \mathrm{RIU}$. The FSR is over $80 \mathrm{~nm}$ which allows our sensor to measure a wider range of refractive indices and hence provides high potential in the fields of bio-sensing and other environmental applications.

As a comparison in the two types of sensors, one can easily find out that the type 2 microfiber sensor outperforms type 1 microfiber sensor in terms of sensitivity, ease of fabrication, cost-effectiveness etc. By optimizing type 2 microfiber sensor in its sensitivity and measuring range, its performance in liquid environment was shown to be promising.

In addition, the optical microfiber sensors that was functionalized with chelating agents to detect specific metal ions in water samples were investigated. The issues 
of the cross sensitivity caused by strain and temperature was resolved by using a proper cartridge design and strain controller. The chelating agents such as EDTA and DPA were successfully coated onto the microfiber surface with a diameter of 3.9 $\mu \mathrm{m}$. The detection limits for metal ions including Lead (II), Copper (II), Zinc (II) and Cadmium (II) ions were ranging from 10-100 ppb. The association of the metal ions and the chelating agents was characterized by EDX. Such compact design and miniaturized sensing region could potentially replace the current bulky and expensive water quality detection systems.

\subsection{Recommendations for future work}

To further explore both types of the microfiber sensors, several suggestions are listed as follows.

1. For type 1 (adiabatic microfiber) sensor, inscribing Bragg gratings into the microfiber is one feasible way for sensing the refractive index. However, since the Bragg gratings do not scatter light into claddings, the evanescent field exposed to surroundings is weak so that the RI sensitivity of microfiber Bragg grating sensor does not have much room for enhancement. However, for microfiber long-period gratings, the sensitivity can still be further improved by reducing the diameter of the microfiber. The issue that needs to be addressed is that the germanium doping inside the fiber core will disperse to claddings during fabrication of microfiber, which makes it very difficult to inscribe long-period gratings into the microfiber. The smaller diameter of the microfiber, the more dispersion of the germanium doping. The possible 
way to resolve this issue will be using highly doped photosensitive fiber for fabrication of microfiber. After the microfiber is drawn, it was placed in hydrogen loader for a long period (over 200 hours). In this way, the loss of the fiber core's photosensitivity during tapering can be compensated after hydrogen loading. Therefore, further reducing the microfiber's diameter to achieve greater sensitivity becomes possible. Besides grating assisted microfiber sensor, surface plasmon resonance in gold or silver coated fibers can also be adopted. The enhancement of the evanescent field could greatly help on detecting the surface changes of the microfiber.

2. For type 2 (non-adiabatically tapered microfiber) sensor, the performance was proven to be great in chapters 4 and 5. However, although the RI sensitivity is extraordinary compared with most of the current optical fiber RI sensors, the cross-sensitivity is also one of major issues that need to be resolved. Owing to the existence of strong evanescent field of such sensor, it is very sensitive to not only RI, but also strain and temperature. In the characterization process, the fluctuation of the strain applied in the microfiber and change in surrounding temperature can sometimes greatly impact the results. Therefore, the optimization of such sensors in terms of minimizing the cross-sensitivities caused by strain and temperature will be one of the subjects to be further researched on.

3. The preliminary result of applying type 2 microfiber sensor into applications such as heavy metal ions detection in water samples has been shown to be promising in chapters 6 and 7. However, if the experiment set-up is bulky 
and relies on the steady environment in lab, there is no obvious advantage compared to current systems for heavy metal ions detection. In order to make such sensors applicable for onsite detection in reservoir or some other water sources, designing a compact detection circuit and reader unit to make the entire sensing system into a small and portable device will be carried out. 


\section{Author's publications}

\section{Journal papers:}

1. W. B. Ji, H. H. Liu, S. C. Tjin, K. K. Chow, and A. Lim, "Ultrahigh sensitivity refractive index sensor based on optical microfiber," IEEE Photon. Technol. Lett., vol. 24, pp. 1872-1874, Oct 2012.

2. W. B. Ji, S. C. Tjin, B. Lin, and C. L. Ng, "Highly sensitive refractive index sensor based on adiabatically tapered microfiber long period gratings," Sensors, vol. 13, pp. 14055-14063, Oct 2013.

3. W. B. Ji, Y. C. Tan, B. Lin, S. C. Tjin, and K. K. Chow, "Non-adiabatically tapered microfiber sensor with ultra-short waist," IEEE Photon. Technol. Lett., vol. 26, pp. 2303-2306, November 2014.

4. W. B. Ji, S. H. K. Yap, N. Panwar, B. Lin, K. T. Yong, S. C. Tjin, W. J. Ng, M. A. Majid, "Rapid detection of low-concentration heavy metal ions using optical microfiber sensor," Sensors and Actuators B: Chemical, vol. 237, pp. 142-149, June 2016.

5. Y. C. Tan, W. B. Ji, V. Mamidala, K. K. Chow, S.C. Tjin, "Carbon-nanotubedeposited long period fiber grating for continuous refractive index sensor applications," Sensor. Actuat. B-Chem, vol. 196, pp. 260-264, Jan 2014.

6. A. Lim, W. B. Ji, S. C. Tjin, “Improved Refractive Index Sensitivity Utilizing Long- 
Period Gratings with Periodic Corrugations on Cladding," Journal of Sensors, vol. 2012, pp. 1687-1691, May 2012.

7. Z. F. Wu, Y. G. Liu, Z. Wang, M. Jiang, W. B. Ji, T. T. Han, S. Li, X. G. Shao, X. Q. Dinh, S. C. Tjin, and P. Shum, "Simultaneous measurement of curvature and strain based on fiber Bragg grating in two-dimensional waveguide array fiber," Opt. Lett., vol. 38, pp. 4070-4073,Oct 2013.

\section{Conference paper:}

8. W. B. Ji, B. Lin, S. C. Tjin, Y. P. Liu, and B. Zhang, "Non-adiabatically tapered microfiber refractometric sensors and its potential applications in environmental detection," Advanced Phonics Congress 2014, Spain.

9. B. Lin, W. B. Ji, S. C. Tjin, and J. Z. Hao, "Armored Fiber Bragg Grating Sensor for Wall Intrusion Detection," Frontiers in Optics 2014, U.S.

10. Z. F. Wu, X. G. Shao, P. Shum, T. Y. Huang, W. B. Ji, N. Zhang, Y. Cui, J. Xue, S. C. Tjin, X. Q. Dinh, "Optimizing Birefringence of Polarization-Maintaining Photonic Crystal Fiber," CLEO Applications and Technology, Jan 2014. 


\section{References}

[1] G. Nosenzo, "Innovative use of embedded FBG sensors in civil engineering and other applications," Fiber Optic Sensors and Applications Viii, vol. 8028, 2011.

[2] A. Dhawan and J. F. Muth, "In-line fiber optic structures for environmental sensing applications," Optics Letters, vol. 31, pp. 1391-1393, May 152006.

[3] G. Shambat, S. R. Kothapalli, A. Khurana, J. Provine, T. Sarmiento, K. Cheng, et al., "A photonic crystal cavity-optical fiber tip nanoparticle sensor for biomedical applications," Applied Physics Letters, vol. 100, May 212012.

[4] C. Fernandez-Valdivielso, I. R. Matias, and F. J. Arregui, "Simultaneous measurement of strain and temperature using a Fiber Bragg Grating and a thermochromic material," Ofs 2002: 15th Optical Fiber Sensors Conference Technical Digest, pp. 203-206, 2002.

[5] W. Liang, Y. Y. Huang, Y. Xu, R. K. Lee, and A. Yariv, "Highly sensitive fiber Bragg grating refractive index sensors," Applied Physics Letters, vol. 86, Apr 112005.

[6] S. Lacroix, R. J. Black, C. Veilleux, and J. Lapierre, "Tapered single-mode fibers: external refractive-index dependence," Appl Opt, vol. 25, pp. 2468-9, Aug 1 1986. 
[7] P. Polynkin, A. Polynkin, N. Peyghambarian, and M. Mansuripur, "Evanescent field-based optical fiber sensing device for measuring the refractive index of liquids in microfluidic channels," Opt Lett, vol. 30, pp. 1273-5, Jun 12005.

[8] V. P. K. Khairnar and RS, "Fabrication and numerical evaluation of the tapered single mode optical fiber: Detection of change in refractive index," Indian Journal of Pure \& Applied Physics, vol. 46, pp. 23-29, 2008.

[9] C. Sui, P. Wu, and B. Ye, "Highly sensitive sensor for detecting refractive index change of liquids using single microfiber," in Nanoelectronics Conference (INEC), 2010 3rd International, 2010, pp. 1343-1344.

[10] Z. Tian, S. S. Yam, and H.-P. Loock, "Refractive index sensor based on an abrupt taper Michelson interferometer in a single-mode fiber," Optics letters, vol. 33, pp. 1105-1107, 2008.

[11] Z. Tian, S. S.-H. Yam, J. Barnes, W. Bock, P. Greig, J. M. Fraser, et al., "Refractive index sensing with Mach-Zehnder interferometer based on concatenating two single-mode fiber tapers," Photonics Technology Letters, IEEE, vol. 20, pp. 626-628, 2008.

[12] J. Yang, L. Jiang, S. Wang, B. Li, M. Wang, H. Xiao, et al., "High sensitivity of taper-based Mach-Zehnder interferometer embedded in a thinned optical fiber for refractive index sensing," Applied optics, vol. 50, pp. 5503-5507, 2011.

[13] N.-K. Chen, T.-H. Yang, Z.-Z. Feng, Y.-N. Chen, and C. Lin, "Cellulardimension picoliter-volume index microsensing using micro-abrupt-tapered fiber 
Mach-Zehnder interferometers," Photonics Technology Letters, IEEE, vol. 24, pp. 842-844, 2012.

[14] P. Wang, G. Brambilla, M. Ding, T. Lee, L. Bo, Y. Semenova, et al., "Enhanced refractometer based on periodically tapered small core singlemode fiber," Sensors Journal, IEEE, vol. 13, pp. 180-185, 2013.

[15] W. B. Ji, H. H. Liu, S. C. Tjin, K. K. Chow, and A. Lim, "Ultrahigh sensitivity refractive index sensor based on optical microfiber," Photonics Technology Letters, IEEE, vol. 24, pp. 1872-1874, 2012.

[16] P. Lu, L. Men, K. Sooley, and Q. Chen, "Tapered fiber Mach-Zehnder interferometer for simultaneous measurement of refractive index and temperature," Applied Physics Letters, vol. 94, p. 131110, 2009.

[17] W. Liang, Y. Huang, Y. Xu, R. K. Lee, and A. Yariv, "Highly sensitive fiber Bragg grating refractive index sensors," Applied Physics Letters, vol. 86, p. 151122, 2005.

[18] Y. Zhang, B. Lin, S. C. Tjin, H. Zhang, G. Wang, P. Shum, et al., "Refractive index sensing based on higher-order mode reflection of a microfiber Bragg grating," Optics express, vol. 18, pp. 26345-26350, 2010.

[19] Y. Ran, Y.-N. Tan, L.-P. Sun, S. Gao, J. Li, L. Jin, et al., "193nm excimer laser inscribed Bragg gratings in microfibers for refractive index sensing," Optics express, vol. 19, pp. 18577-18583, 2011. 
[20] G. Wang, P. P. Shum, H.-p. Ho, X. Yu, D. J. J. Hu, Y. Cui, et al., "Modeling and analysis of localized biosensing and index sensing by introducing effective phase shift in microfiber Bragg grating ( $\mu$ FBG)," Optics express, vol. 19, pp. 8930-8938, 2011.

[21] Y. Liu, C. Meng, A. P. Zhang, Y. Xiao, H. Yu, and L. Tong, "Compact microfiber Bragg gratings with high-index contrast," Optics letters, vol. 36, pp. 3115-3117, 2011

[22] Y. Ran, L. Jin, L.-P. Sun, J. Li, and B.-O. Guan, "Bragg gratings in rectangular microfiber for temperature independent refractive index sensing," Optics letters, vol. 37, pp. 2649-2651, 2012.

[23] J.-F. Ding, L.-Y. Shao, J.-H. Yan, and S. He, "Fiber-taper seeded long-period grating pair as a highly sensitive refractive-index sensor," Photonics Technology Letters, IEEE, vol. 17, pp. 1247-1249, 2005.

[24] G. Cardenas-Sevilla, D. Monzon-Hernandez, I. Torres-Gomez, and A. Martínez-Ríos, "Tapered Mach-Zehnder interferometer based on two mechanically induced long-period fiber gratings as refractive index sensor," Optics \& Laser Technology, vol. 44, pp. 1516-1520, 2012.

[25] T. Allsop, F. Floreani, K. Jedrzejewski, P. Marques, R. Romero, D. Webb, et al., "Refractive index sensing with long-period grating fabricated in biconical tapered fibre," Electronics letters, vol. 41, pp. 471-472, 2005. 
[26] P. Wu, C. Sui, and B. Ye, "Modelling nanofiber Mach-Zehnder interferometers for refractive index sensors," Journal of Modern Optics, vol. 56, pp. 2335-2339, 2009.

[27] A. Diez, M. Andres, and J. Cruz, "In-line fiber-optic sensors based on the excitation of surface plasma modes in metal-coated tapered fibers," Sensors and Actuators B: Chemical, vol. 73, pp. 95-99, 2001.

[28] F. J. Bueno, O. Esteban, N. Diaz-Herrera, M. C. Navarrete, and A. GonzalezCano, "Sensing properties of asymmetric double-layer-covered tapered fibers," Appl Opt, vol. 43, pp. 1615-20, Mar 102004.

D. Monzón-Hernández and J. Villatoro, "High-resolution refractive index sensing by means of a multiple-peak surface plasmon resonance optical fiber sensor," Sensors and Actuators B: Chemical, vol. 115, pp. 227-231, 2006.

[30] Y. Cui, P. P. Shum, G. Wang, H. Chang, X. Q. Dinh, M. Jiang, et al., "Size effect of gold nanoparticles on optical microfiber refractive index sensors," in Sensors, 2011 IEEE, 2011, pp. 371-374.

[31] G. Liu, Y. Wu, K. Li, P. Hao, P. Zhang, and M. Xuan, "Mie scatteringenhanced fiber-optic refractometer," Photonics Technology Letters, IEEE, vol. 24, pp. 658-660, 2012.

[32] H.-Y. Lin, C.-H. Huang, G.-L. Cheng, N.-K. Chen, and H.-C. Chui, "Tapered optical fiber sensor based on localized surface plasmon resonance," Optics express, vol. 20, pp. 21693-21701, 2012. 
[33] S. J. Qiu, Y. Chen, J. L. Kou, F. Xu, and Y. Q. Lu, "Miniature tapered photonic crystal fiber interferometer with enhanced sensitivity by acid microdroplets etching," Appl Opt, vol. 50, pp. 4328-32, Aug 12011.

[34] Q. Liu and Q. Wang, "Refractive index sensor based on tapered PCF in-line interferometer," Chinese Optics Letters, vol. 10, pp. 090601-090601, 2012.

[35] C. Li, S.-J. Qiu, Y. Chen, F. Xu, and Y.-Q. Lu, "Ultra-sensitive refractive index sensor with slightly tapered photonic crystal fiber," IEEE Photonics Technology Letters, vol. 24, p. 1771, 2012.

[36] P. Wang, G. Brambilla, M. Ding, Y. Semenova, Q. Wu, and G. Farrell, "High-sensitivity, evanescent field refractometric sensor based on a tapered, multimode fiber interference," Optics letters, vol. 36, pp. 2233-2235, 2011. C. R. Biazoli, S. Silva, M. A. Franco, O. Frazao, and C. M. Cordeiro, "Multimode interference tapered fiber refractive index sensors," Appl Opt, vol. 51, pp. 5941-5, Aug 202012.

[38] G. Y. Chen, M. Ding, T. P. Newson, and G. Brambilla, "A review of microfiber and nanofiber based optical sensors," Open Optic. J, vol. 7, pp. 32-57, 2013.

[39] W. B. Ji, H. H. Liu, S. C. Tjin, K. K. Chow, and A. Lim, "Ultrahigh Sensitivity Refractive Index Sensor Based on Optical Microfiber," Ieee Photonics Technology Letters, vol. 24, pp. 1872-1874, Oct 152012. 
[40] A. Stiebeiner, R. Garcia-Fernandez, and A. Rauschenbeutel, "Design and optimization of broadband tapered optical fibers with a nanofiber waist," Opt Express, vol. 18, pp. 22677-85, Oct 252010.

[41] L. Tong, L. Hu, J. Zhang, J. Qiu, Q. Yang, J. Lou, et al., "Photonic nanowires directly drawn from bulk glasses," Opt Express, vol. 14, pp. 82-7, Jan 92006.

[42] G. Brambilla, F. Koizumi, X. Feng, and D. Richardson, "Compound-glass optical nanowires," Electronics Letters, vol. 41, pp. 400-402, 2005.

[43] E. C. Magi, L. B. Fu, H. C. Nguyen, M. R. Lamont, D. I. Yeom, and B. J. Eggleton, "Enhanced Kerr nonlinearity in sub-wavelength diameter $\operatorname{As}(2) \operatorname{Se}(3)$ chalcogenide fiber tapers," Opt Express, vol. 15, pp. 10324-9, Aug 62007.

[44] S. A. Harfenist, S. D. Cambron, E. W. Nelson, S. M. Berry, A. W. Isham, M. M. Crain, et al., "Direct drawing of suspended filamentary micro-and nanostructures from liquid polymers," Nano Letters, vol. 4, pp. 1931-1937, 2004.

[45] A. S. Nain, J. C. Wong, C. Amon, and M. Sitti, "Drawing suspended polymer micro-/nanofibers using glass micropipettes," Applied physics letters, vol. 89, p. $183105,2006$.

[46] F. Gu, L. Zhang, X. Yin, and L. Tong, "Polymer single-nanowire optical sensors," Nano Lett, vol. 8, pp. 2757-61, Sep 2008. 
[47] X. Xing, Y. Wang, and B. Li, "Nanofibers drawing and nanodevices assembly in poly (trimethylene terephthalate)," Optics express, vol. 16, pp. 1081510822, 2008.

[48] K. Okamoto, Fundamentals of optical waveguides: Academic press, 2010.

[49] L. Tong, J. Lou, and E. Mazur, "Single-mode guiding properties of subwavelength-diameter silica and silicon wire waveguides," Opt Express, vol. 12, pp. 1025-35, Mar 222004.

[50] M. Wu, W. Huang, and L. Wang, "Propagation characteristics of the silica and silicon subwavelength-diameter hollow wire waveguides," Chinese Optics Letters, vol. 6, pp. 732-735, 2008.

[51] J. D. Love, W. M. Henry, W. J. Stewart, R. J. Black, S. Lacroix, and F. Gonthier, "Tapered single-mode fibres and devices. I. Adiabaticity criteria," Optoelectronics, IEE Proceedings J, vol. 138, pp. 343-354, 1991.

[52] J. D. Love, "Application of a low-loss criterion to optical waveguides and devices," Optoelectronics, IEE Proceedings J, vol. 136, pp. 225-228, 1989.

[53] R. J. Black, S. Lacroix, F. Gonthier, and J. D. Love, "Tapered single-mode fibres and devices. II. Experimental and theoretical quantification," Optoelectronics, IEE Proceedings J, vol. 138, pp. 355-364, 1991.

[54] W. M. Henry, Tapered Optical Waveguides and Metal-clad Polarisers: Australian National University, 1988. 
[55] A. W. Snyder and J. Love, Optical Waveguide Theory: Springer, 1983.

[56] L. Tong and M. Sumetsky, Subwavelength and nanometer diameter optical fibers: Springer Science \& Business Media, 2011.

[57] L. Tong, R. R. Gattass, J. B. Ashcom, S. He, J. Lou, M. Shen, et al., "Subwavelength-diameter silica wires for low-loss optical wave guiding," Nature, vol. 426, pp. 816-819, 2003.

[58] G. Brambilla, V. Finazzi, and D. Richardson, "Ultra-low-loss optical fiber nanotapers," Optics Express, vol. 12, pp. 2258-2263, 2004.

[59] S. Leon-Saval, T. Birks, W. Wadsworth, P. St J Russell, and M. Mason, "Supercontinuum generation in submicron fibre waveguides," Optics Express, vol. 12, pp. 2864-2869, 2004.

[60] L. Tong, J. Lou, Z. Ye, G. T. Svacha, and E. Mazur, "Self-modulated taper drawing of silica nanowires," Nanotechnology, vol. 16, p. 1445, 2005.

[61] S. Pricking and H. Giessen, "Tapering fibers with complex shape," Optics express, vol. 18, pp. 3426-3437, 2010.

[62] H. Xuan, J. Ju, and W. Jin, "Highly birefringent optical microfibers," Optics express, vol. 18, pp. 3828-3839, 2010.

[63] L. Shi, X. Chen, H. Liu, Y. Chen, Z. Ye, W. Liao, et al., "Fabrication of submicron-diameter silica fibers using electric strip heater," Optics express, vol. 14, pp. 5055-5060, 2006. 
[64] A. Coillet, B. Cluzel, G. Vienne, P. Grelu, and F. de Fornel, "Near-field characterization of glass microfibers on a low-index substrate," Applied Physics B, vol. 101, pp. 291-295, 2010.

[65] T. E. Dimmick, G. Kakarantzas, T. A. Birks, and P. S. J. Russell, "Carbon dioxide laser fabrication of fused-fiber couplers and tapers," Applied Optics, vol. 38, pp. 6845-6848, 1999.

[66] M. Sumetsky, Y. Dulashko, and A. Hale, "Fabrication and study of bent and coiled free silica nanowires: Self-coupling microloop optical interferometer," Optics Express, vol. 12, pp. 3521-3531, 2004.

[67] P. Pal and W. H. Knox, "Low loss fusion splicing of micron scale silica fibers," Optics express, vol. 16, pp. 11568-11573, 2008.

[68] P. Wang, L. Zhang, Z. Yang, F. Gu, S. Wang, Q. Yang, et al., "Fusion spliced microfiber closed-loop resonators," IEEE Photonics Technology Letters, vol. 22, pp. 1075-1077, 2010.

[69] Z. Tian, M. Nix, and S. S. Yam, "Laser beam shaping using a single-mode fiber abrupt taper," Optics letters, vol. 34, pp. 229-231, 2009.

[70] L. Ç. Özcan, V. Treanton, F. Guay, and R. Kashyap, "Highly Symmetric Optical Fiber Tapers Fabricated With a CO 2 Laser," Photonics Technology Letters, IEEE, vol. 19, pp. 656-658, 2007. 
[71] J. Lou, Y. Wang, and L. Tong, "Microfiber optical sensors: A review," Sensors, vol. 14, pp. 5823-5844, 2014.

[72] G. Keiser, F. Xiong, Y. Cui, and P. P. Shum, "Review of diverse optical fibers used in biomedical research and clinical practice," Journal of biomedical optics, vol. 19, pp. 080902-080902, 2014.

[73] F. Bilodeau, K. O. Hill, B. Malo, D. C. Johnson, and I. M. Skinner, "Efficient, narrowband LP01LP02 mode convertors fabricated in photosensitive fibre: spectral response," Electronics Letters, vol. 27, pp. 682-4, 04/11 1991.

[74] R. Kashyap, Fiber Bragg Gratings: Elsevier Academic Press, 1999.

[75] A. Othonos and K. Kalli, Fiber Bragg gratings : fundamentals and applications in telecommunications and sensing. Boston, Mass.: Artech House, 1999.

[76] A. D. Kersey, M. A. Davis, H. J. Patrick, M. LeBlanc, K. P. Koo, C. G. Askins, et al., "Fiber grating sensors," Journal of Lightwave Technology, vol. 15, pp. 1442-1463, Aug 1997.

[77] L. Wei, H. Yanyi, X. Yong, R. K. Lee, and A. Yariv, "Highly sensitive fiber Bragg grating refractive index sensors," Applied Physics Letters, vol. 86, p. 151122 (3 pp.), 04/11 2005.

[78] A. Iadicicco, A. Cusano, A. Cutolo, R. Bernini, and M. Giordano, "Thinned fiber Bragg gratings as high sensitivity refractive index sensor," IEEE Photonics Technology Letters, vol. 16, pp. 1149-51, 04/ 2004. 
[79] Y. Zhang, B. Lin, S. C. Tjin, H. Zhang, G. H. Wang, P. Shum, et al., "Refractive index sensing based on higher-order mode reflection of a microfiber Bragg grating," Optics Express, vol. 18, pp. 26345-26350, Dec 62010.

[80] H. F. Taylor and A. Yariv, "Guided Wave Optics," Proceedings of the Ieee, vol. 62, pp. 1044-1060, 1974.

[81] A. Othonos, "Fiber Bragg gratings," Review of Scientific Instruments, vol. 68, pp. 4309-4341, Dec 1997.

[82] D. L. Williams, B. J. Ainslie, J. R. Armitage, R. Kashyap, and R. Campbell, "Enhanced Uv Photosensitivity in Boron Codoped Germanosilicate Fibers," Electronics Letters, vol. 29, pp. 45-47, Jan 71993.

[83] S. W. James and R. P. Tatam, "Optical fibre long-period grating sensors: characteristics and application," Measurement science and technology, vol. 14, pp. R49-R61, 2003.

[84] R. Hou, Z. Ghassemlooy, A. Hassan, C. Lu, and K. Dowker, "Modelling of long-period fibre grating response to refractive index higher than that of cladding," Measurement Science and Technology, vol. 12, p. 1709, 2001.

[85] O. Duhem, J.-F. Henninot, M. Warenghem, and M. Douay, "Demonstration of long-period-grating efficient couplings with an external medium of a refractive index higher than that of silica," Applied optics, vol. 37, pp. 7223-7228, 1998. 
[86] M. N. Ng and K. S. Chiang, "Thermal effects on the transmission spectra of long-period fiber gratings," Optics Communications, vol. 208, pp. 321-327, Jul 15 2002.

[87] J. N. Jang, S. Y. Kim, S. W. Kim, and M. S. Kim, "Temperature insensitive long-period fibre gratings," Electronics Letters, vol. 35, pp. 2134-2136, Nov 251999.

[88] V. Bhatia, "Applications of long-period gratings to single and multiparameter sensing," Optics Express, vol. 4, pp. 457-466, May 241999.

[89] X. W. Shu, T. Allsop, B. Gwandu, L. Zhang, and I. Bennion, "Hightemperature sensitivity of long-period gratings in B-Ge codoped fiber," Ieee Photonics Technology Letters, vol. 13, pp. 818-820, Aug 2001.

[90] K. Q. Kieu and M. Mansuripur, "Biconical fiber taper sensors," Ieee Photonics Technology Letters, vol. 18, pp. 2239-2241, Nov-Dec 2006.

[91] P. F. Wang, G. Brambilla, M. Ding, Y. Semenova, Q. Wu, and G. Farrell, "High-sensitivity, evanescent field refractometric sensor based on a tapered, multimode fiber interference," Optics Letters, vol. 36, pp. 2233-2235, Jun 152011.

[92] G. Salceda-Delgado, D. Monzon-Hernandez, A. Martinez-Rios, G. A. Cardenas-Sevilla, and J. Villatoro, "Optical microfiber mode interferometer for temperature-independent refractometric sensing," Optics Letters, vol. 37, pp. 19741976, Jun 12012. 
[93] S. Lacroix, F. Gonthier, R. J. Black, and J. Bures, "Tapered-Fiber Interferometric Wavelength Response - the Achromatic Fringe," Optics Letters, vol. 13, pp. 395-397, May 1988.

[94] D. T. Cassidy, D. C. Johnson, and K. O. Hill, "Wavelength-Dependent Transmission of Monomode Optical Fiber Tapers," Applied Optics, vol. 24, pp. 945$950,1985$.

[95] A. W. Snyder and J. D. Love, Optical waveguide theory. London ; New York: Chapman and Hall, 1983.

[96] Y. Li, L. A. Chen, E. Harris, and X. Y. Bao, "Double-Pass In-Line Fiber Taper Mach-Zehnder Interferometer Sensor," Ieee Photonics Technology Letters, vol. 22, pp. 1750-1752, Dec 12010.

[97] R. Yang, Y. S. Yu, Y. Xue, C. Chen, Q. D. Chen, and H. B. Sun, "Single Stapered fiber Mach-Zehnder interferometers," Optics Letters, vol. 36, pp. 4482-4484, Dec 12011.

[98] E. Hecht, "Optics 2nd edition," Optics 2nd edition by Eugene Hecht Reading, MA: Addison-Wesley Publishing Company, 1987, vol. 1, 1987.

[99] E. Hecht, "Optics, 4th," International edition, Addison-Wesley, San Francisco, pp. 467-473, 2002. 
[100] Y. Xue, Y. S. Yu, R. Yang, C. Wang, C. Chen, J. C. Guo, et al., "Ultrasensitive temperature sensor based on an isopropanol-sealed optical microfiber taper," Optics Letters, vol. 38, pp. 1209-1211, Apr 152013.

[101] C. L. Du and X. H. Yang, "Highly Sensitive Refractive Index Measurement Based an a Thinned Fiber Taper," Microwave and Optical Technology Letters, vol. 56, pp. 1054-1057, May 2014.

[102] J. L. Kou, J. Feng, Q. J. Wang, F. Xu, and Y. Q. Lu, "Microfiber-probe-based ultrasmall interferometric sensor," Opt Lett, vol. 35, pp. 2308-10, Jul 12010.

[103] R. Singh, N. Gautam, A. Mishra, and R. Gupta, "Heavy metals and living systems: An overview," Indian J Pharmacol, vol. 43, pp. 246-53, May 2011.

[104] H. W. Chen, "Gallium, indium, and arsenic pollution of groundwater from a semiconductor manufacturing area of Taiwan," Bull Environ Contam Toxicol, vol. 77, pp. 289-96, Aug 2006.

[105] T. Roychowdhury, H. Tokunaga, and M. Ando, "Survey of arsenic and other heavy metals in food composites and drinking water and estimation of dietary intake by the villagers from an arsenic-affected area of West Bengal, India," Sci Total Environ, vol. 308, pp. 15-35, Jun 12003.

[106] C. R. Xing, L. Q. Liu, X. Zhang, H. Kuang, and C. L. Xu, "Colorimetric detection of mercury based on a strip sensor," Analytical Methods, vol. 6, pp. 62476253, Aug 212014. 
[107] Y. Ning, K. Grattan, W. Wang, and A. Palmer, "A systematic classification and identification of optical fibre sensors," Sensors and Actuators A: Physical, vol. 29, pp. 21-36, 1991.

[108] P. K. Jal, S. Patel, and B. Mishra, "Chemical modification of silica surface by immobilization of functional groups for extractive concentration of metal ions," Talanta, vol. 62, pp. 1005-1028, Apr 192004.

[109] B. B. Gu, M. J. Yin, A. P. Zhang, J. W. Qian, and S. L. He, "Fiber-optic metal ion sensor based on thin-core fiber modal interferometer with nanocoating selfassembled via hydrogen bonding," Sensors and Actuators B-Chemical, vol. 160, pp. 1174-1179, Dec 152011.

[110] M. Benounis, N. Jaffrezic-Renault, H. Halouani, R. Lamartine, and I. Dumazet-Bonnamour, "Detection of heavy metals by an optical fiber sensor with a sensitive cladding including a new chromogenic calix[4]arene molecule," Materials Science \& Engineering C-Biomimetic and Supramolecular Systems, vol. 26, pp. 364368, Mar 2006.

[111] Y. Gong, C. B. Yu, T. T. Wang, X. P. Liu, Y. Wu, Y. J. Rao, et al., "Highly sensitive force sensor based on optical microfiber asymmetrical Fabry-Perot interferometer," Opt Express, vol. 22, pp. 3578-84, Feb 102014.

[112] W. B. Ji, Y. C. Tan, B. Lin, S. C. Tjin, and K. K. Chow, "Nonadiabatically Tapered Microfiber Sensor With Ultrashort Waist," Ieee Photonics Technology Letters, vol. 26, pp. 2303-2306, Nov 152014. 
[113] J. H. Park, E. J. Hill, T. H. Chou, V. LeQuire, R. Roelofs, and C. R. Park, "Mechanism of action of penicillamine in the treatment of avian muscular dystrophy," Ann N Y Acad Sci, vol. 317, pp. 356-69, 1979.

[114] S. R. Gooneratne and D. A. Christensen, "Effect of chelating agents on the excretion of copper, zinc and iron in the bile and urine of sheep," Vet J, vol. 153, pp. 171-8, Mar 1997. 